Check for updates

Cite this: RSC Adv., 2021, 11, 13409

\section{White light employing luminescent engineered large (mega) Stokes shift molecules: a review}

\author{
Nadia Nabihah Mohd Yusof Chan, (D) ab Azila Idris, ${ }^{\text {aa }}$ Zul Hazrin Zainal Abidin, ${ }^{\mathrm{b}}$ \\ Hairul Anuar Tajuddin ${ }^{a}$ and Zanariah Abdullah ${ }^{a}$
}

Large (mega) Stokes shift molecules have shown great potential in white light emission for optoelectronic applications, such as flat panel display technology, light-emitting diodes, photosensitizers, molecular probes, cellular and bioimaging, and other applications. This review aims to summarize recent developments of white light generation that incorporate a large Stokes shift component, key approaches to designing large Stokes shift molecules, perspectives on future opportunities, and remaining challenges confronting this emerging research field. After a brief introduction of feasible pathways in generating white light, exemplifications of large Stokes shift molecules as white light candidates from organic and inorganic-based materials are illustrated. Various possible ways to design such molecules have been revealed by integrating the photophysical mechanisms that are essential to produce red-shifted emission upon photoexcitation, such as excited state intramolecular proton transfer (ESIPT), intramolecular charge transfer (ICT), excited state geometrical relaxation or structural deformation, aggregation-induced emission (AIE) alongside the different formations of aggregates, interplay between monomer and excimer emission, host-guest interaction, and lastly metal to ligand charge transfer (MLCT) via harvesting triplet state. Furthermore, previously reported fluorescent materials are described and categorized based on luminescence behaviors on account of the Stokes shifts value. This review will serve as a rationalized introduction and reference for researchers who are interested in exploring large or mega Stokes shift molecules, and will motivate new strategies along with instigation of persistent efforts in this prominent subject area with great avenues.
Received 7th January 2021 Accepted 3rd March 2021 DOI: $10.1039 / \mathrm{d} 1 \mathrm{ra00129a}$ rsc.li/rsc-advances

\section{Introduction}

White light can be generated from the combination of the whole visible spectrum of electromagnetic radiation. It can thus appear as broadband or multi-band emission. Physical means to generate white light may involve the combination of a few specific kinds of light, such as compact and discrete red-green-

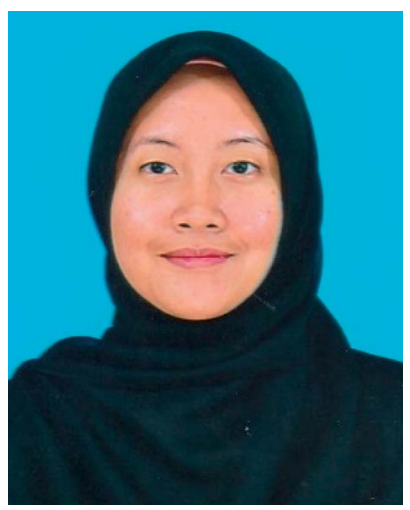

molecules.
Nadia Nabihah Mohd Yusof Chan received her BSc degree in Chemistry from University of Malaya, Malaysia in 2018. Since 2019, she has been a doctoral student in the Department of Chemistry, University of Malaya. Her research interest includes the synthesis of fluorescent materials for white light emission, Stokes shift, and detailed analysis on the photoluminescence properties of

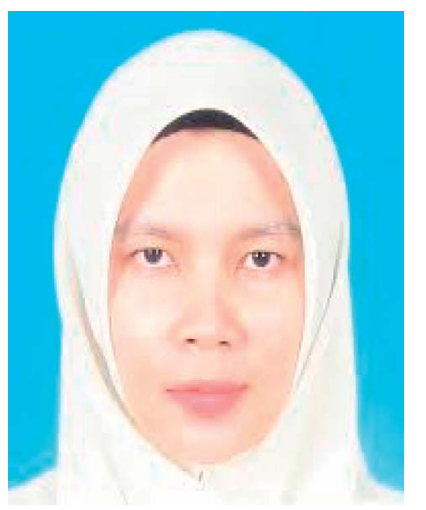

Dr Azila Idris received her PhD degree from Monash University, Australia in 2014. She is currently a Senior Lecturer in the Department of Chemistry, University of Malaya, Malaysia. Her research focuses on biomass utilisation (protein and lignocellulosic), synthesis of heterocyclic compounds and fluorescence studies. 
blue (RGB) light-emitting diodes (LEDs), single phosphor based white LED ${ }^{1}$ uniform photonic crystal fiber using microchip laser, ${ }^{2}$ lanthanides-doped film by pulsed laser ablation ${ }^{3}$ or through the fabrication of multiphase phosphors on an ultraviolet-emitting diode. ${ }^{4}$ Based on the former pathway, the generation of white light resulted from the process of color additive mixing. Upon additive mixing, the color rendering has been evaluated based on the physiologically perceived colors by humans, known as the Commission Internationale d'Eclairage (CIE)-1931 chromaticity diagram. ${ }^{5}$ A pure and ideal white light is designated as $(0.33,0.33)$ in CIE coordinates (Fig. 1$).{ }^{6}$ In the last century, tremendous advancements of white light generation have been witnessed through varying methods and materials. Methods ranged from an attempt for a complementary mixing of colors (blue and yellow or cyan and orange), assembling three primary colors (RGB) in a device, while operating each of them independently, to the utilization of blue or UVLED to excite phosphor materials by means of energy conversion. The most remarkable method to achieve a true white light is reflected upon the correct ratio mixture of three primary colors. ${ }^{7}$ In view of this, researchers developed a single white emissive molecule, ${ }^{8}$ or combined molecules with different emissive wavelengths in a single mixture. ${ }^{\mathbf{9}, 10}$ One of the unwelcomed natures when mixing different molecules, however, is the Förster Resonance Energy Transfer (FRET) effect. The FRET

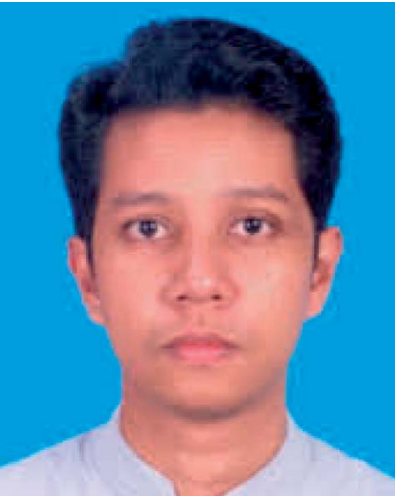

Dr Zul Hazrin Zainal Abidin received his $P h D$ degree in Advanced Materials from the Department of Physics, University of Malaya in 2006. He is now an Associate Professor in the Faculty of Science, University of Malaya. His current research is focused on dyes and pigments, coatings, materials in dyesensitised solar cells, redox mediator, and gel polymer electrolytes applications.

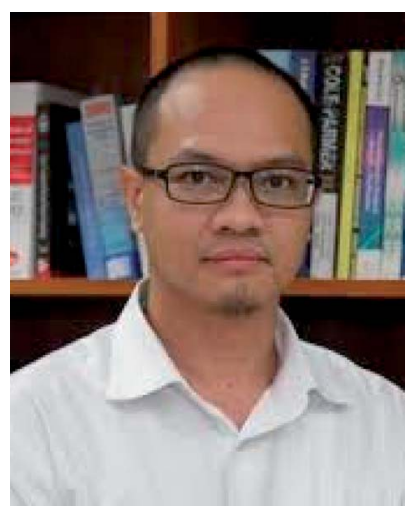

Dr Hairul Anuar Tajuddin received his BSc and $M S c$ degrees from the Department of Chemistry, University of Malaya, and PhD degree from the University of Sheffield, United Kingdom. He became a member of the Faculty of Science, University of Malaya since 2007, and is currently an Associate Professor. His research work involves physical organic chemistry, heterocyclic amphiphiles, organic dyes, organic emitters and colorimetric sensors. effect quenched the emission intensity; thus, curtailing the efficiency and brightness of the light. ${ }^{11}$ Looking from the perspective of white light emitting materials, the photophysical mechanisms need to be scrutinized in order to understand the possible pathways of suitable chromophores designation. ${ }^{12} \mathrm{~A}$ detailed understanding of these mechanisms prompted not only a true white light in the future, but also great photostability, tunability and quantum efficiencies.

The spectral distributions and quantum efficiencies of chromophores are one of the many crucial attributes to be assessed when considering a larger scale material production. It is without doubt that previous research strides have been

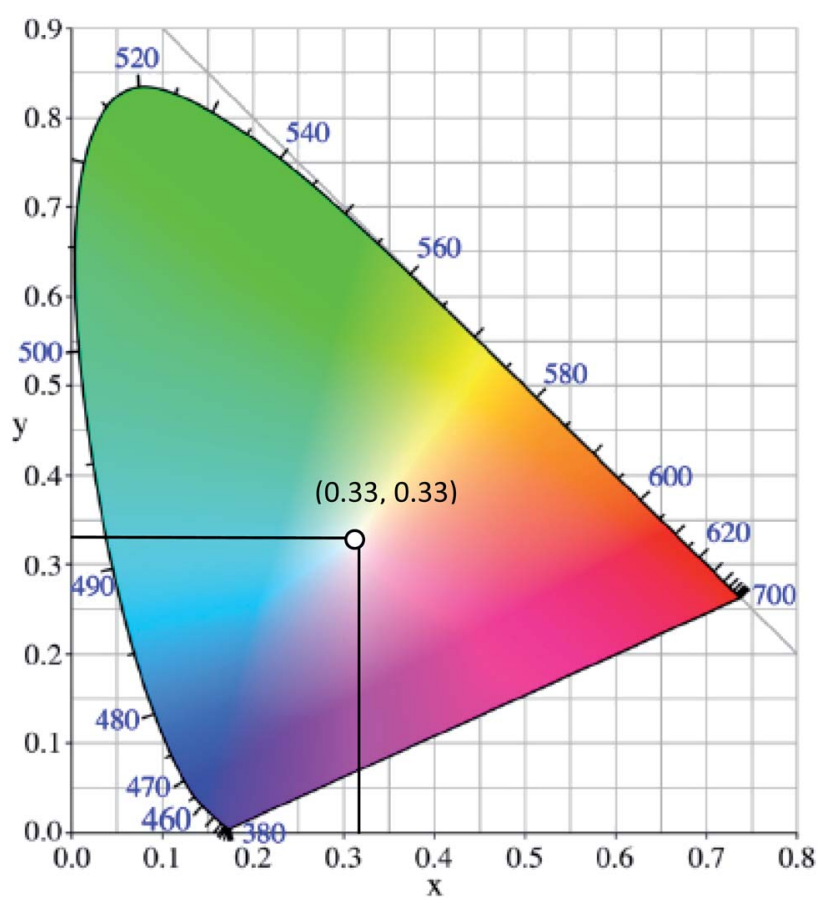

Fig. 1 The chromaticity coordinates $(x, y)$ of pure white light, as given by the Commission Internationale d'Eclairage, is $(0.33,0.33)$. The figure was adapted from ref. 25 with accreditation given to Creative Commons Attribution 4.0 International Public License.

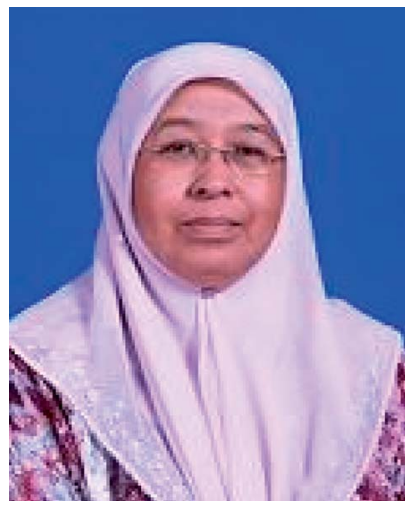

Professor Zanariah Abdullah received her Doctoral Degree from Queen Mary, University of London, United Kingdom in 1989. She joined the Faculty of Science, University of Malaya since 1989, and took up her current position in 2000. Her research interest involves the synthesis and study of luminescent materials and heterocyclic compounds for various applications, including molecular probes and sensors, white light emitter and bioactivities. 
focused on molecules engineered with high quantum efficiencies in their respective emission wavelength region. ${ }^{13-15}$ High quantum efficiency materials are a desirable characteristic in most imaging applications for the best display, illumination, and performance. In white light application, several efforts to design suitable chromophores have been done by different research groups, employing organic, ${ }^{\mathbf{9}}$ inorganic, ${ }^{\mathbf{1 6}}$ supramolecular chemistry, ${ }^{\mathbf{1 7}}$ polymer micelle, ${ }^{\mathbf{1 8}, \mathbf{1 9}}$ biocomposite, ${ }^{7}$ lanthanides doped phosphors, ${ }^{3,20}$ quantum dot, ${ }^{21}$ carbon dot, ${ }^{22,23}$ and other approaches. Continuous efforts in experimenting with different types of white light emitting materials were inspired by the demand for cost effective production, usage of low toxicity materials, scarcity of resources that make up current materials, facile fabrication, green strategy and existing loopholes in generating a true white light. ${ }^{24}$ The discussion in this review article highlights the recent developments of employing large Stokes shift materials to generate white light, diverse classes of organic and inorganic materials with large Stokes shift, and molecular engineering approaches to large Stokes shift molecules with relevant applicability to optoelectronic devices. Practically, a large or mega Stokes shift is a desired feature for fluorophores in white light emission to inhibit self-absorption or the inner filter effect.

\section{Employing large Stokes shift molecules in white light emission}

An ideal white light emitter requires the simultaneous emission of red, green, and blue with almost similar intensity distribution to cover the entire region of the visible spectrum. On this basis, research groups of vastly different expertise have addressed the fundamental question to fulfill this condition by developing a single white emitter or mixing different emitters that originate from a single excitation wavelength. Through this approach, there will be no or minimal emission reabsorption that would quench the overall white intensity and efficiency. Nevertheless, such strategy is undeniably challenging and requires future extensive study to gain comprehension of the multicomponent mixture behaviors. The realization of a material with multicomponent emitters is expected by connecting them via covalent bonds or noncovalent interactions. ${ }^{26}$ This includes hydrogen bonds, van der Waals forces, $\pi-\pi$ stacking, dipole-induced dipole forces, metal coordination, and others. In order to achieve this, employing a large Stokes shift molecule within the selection of material provides a state-of-the-art strategy that becomes of paramount importance in the field of optoelectronics, bio-imaging, next-generation display and sensing devices.

A Stokes shift is the difference in energy between the band maximum of the absorption and the lower energy emission. It is a key parameter of the luminescence property because its value quantifies and explains other significant parameters of the studied compounds, for instance, how the Stokes shift justifies the different dipole moments of molecules in different electronic states and how changes in the solvent polarity affects the polarization and anisotropy of fluorescence. ${ }^{27}$ The relationship between the solvent polarity parameter and Stokes shift can be evaluated quantitatively according to the Lippert-Mataga equation below.$^{28} \Delta v$ corresponds to the Stokes shift value $\left(\mathrm{cm}^{-1}\right)$, while $v_{\mathrm{a}}$ and $v_{\mathrm{e}}$ are the absorption and emission wavenumbers, respectively. The letter $h$ is Planck's constant, $c$ is the speed of light, and $a$ is the Onsager radius. Parameters $\mu_{\mathrm{e}}$ and $\mu_{\mathrm{g}}$ are the permanent dipole moments of the excited state and ground state, respectively, in which values can be obtained from the slope of a Lippert-Mataga plot. $\varepsilon_{0}$ corresponds to the permittivity in a vacuum, $\varepsilon$ is the static dielectric constant of the solvent, $n$ is the refractive index and $\Delta f$ represents the orientational polarizability. This plot has been reported by researchers when studying the Stokes shift parameter of molecules..$^{29-31}$

$$
\begin{gathered}
\Delta f=\frac{\varepsilon-1}{2 \varepsilon+1}-\frac{n^{2}-1}{2 n^{2}+1} \\
\Delta v=v_{\mathrm{a}}-v_{\mathrm{e}}=\frac{2\left(\mu_{\mathrm{e}}-\mu_{\mathrm{g}}\right)^{2}}{4 \pi \varepsilon_{0} h c a^{3}} \Delta f+\mathrm{const}
\end{gathered}
$$

The importance of the Stokes shift in the phenomena of spectral diffusion and spectral heterogeneity at room temperature has been demonstrated by Stopel and his co-workers in their single organic molecule study. ${ }^{32}$ The Stokes shift has always been assumed to be constant for single molecules. However, in reality, heterogeneity in the conformation of chromophores and their nanoenvironments can determine the exact photophysical quantities, including the lifetime, polarization and Stokes shift variation. In Stopel et al.'s study, the polystyrene-embedded perylene derivative $N, N$-di(tert-butoxycarbonyl)-9-amino- $N$-(2,6-diisopropylphenyl)perylene-3,4-

dicarboximide showed a Stokes shift of $96 \mathrm{meV}$ for the ensemble toluene solution. Meanwhile, for the single molecule analysis, they recorded 80 emission spectra to construct a histogram of the highest energy emission peak energies (Fig. 2). The broad width distribution ( $95 \mathrm{meV}$ ) of the histogram implies inconsistency of the Stokes shift for different molecules, reflecting differences in the polarity and flexibility of the individual nanoenvironments that directly affect the Stokes shift. This study has initiated a promising tool to study the correlation of the energy band gap between the ground state and excited state, besides quantifying the local variations of the chromophores in matrix-like polymers. Future experiments involving single organic dyes in solvents of varying polarity may be conducted to establish a detailed characterization of the Stokes shift.

\subsection{Organic molecules}

After having some insights on the importance of the Stokes shift, the next aspect to consider is the challenges in the synthesis and molecular designation of large Stokes shift materials. Herein, several organic-based molecules that were reported to exhibit a large Stokes shift are to be discussed. There are huge classes of organic compounds that exhibit a large Stokes shift, such as terpyridines, pyrenes, BODIPY, coumarins, benzothiazoles, quinolines, quinoxalines, and others. Studies 

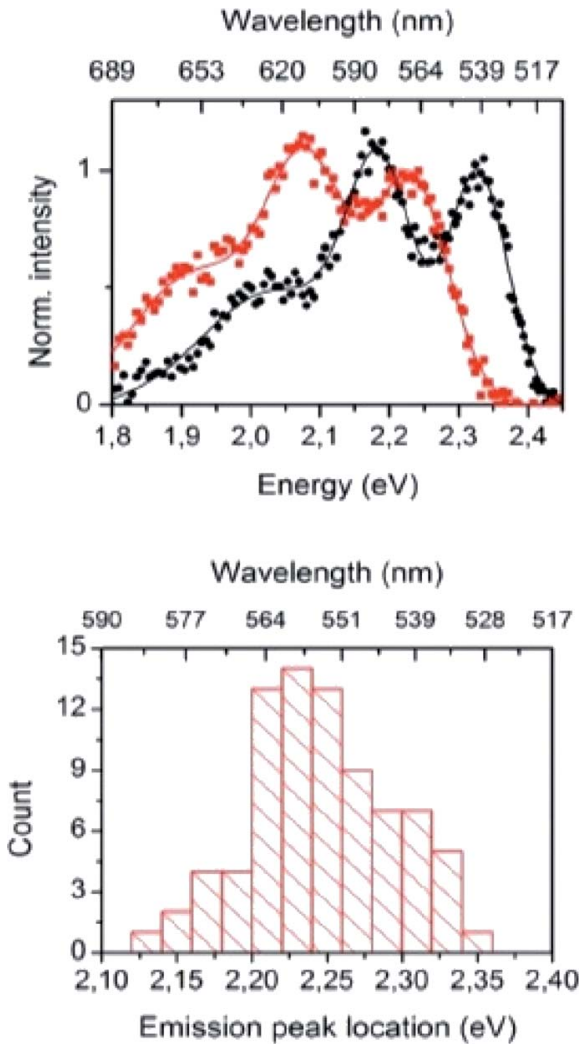

Fig. 2 (Top) Examples of the recorded emission spectra from different emitters, indicating molecule-to-molecule differences in the shape and the spectra position. Solid lines are a guide to the eye, and based on double and triple Gaussian fits to the data. (Bottom) The histogram for the emission shows the distribution of the peak positions. The figure was adapted from ref. 32 with permission. Copyright 2014 American Chemical Society.

revolving around pure organic molecules as emitters started when researchers aspired for a greener approach and low cost production as a replacement for metals, inorganic or biological hybrids. In this section, each structural framework will be reviewed in terms of their photoluminescence properties and potential applicability in white light emission. A hybrid fluorophore based on terpyridine-benzoxadiazole (TPBD) was developed by Yang et al., which enlarged the donor- $\pi$-acceptor system, leading to a large Stokes shift $(127 \mathrm{~nm}) \cdot{ }^{33} \mathrm{In}$ an acidic environment $(\mathrm{pH}=2)$, the absorption wavelength red-shifted from $470 \mathrm{~nm}$ to $494 \mathrm{~nm}$. This was due to the protonation of two lateral pyridine nitrogen atoms, which enhanced the electron-withdrawing ability of the terpyridine moiety. Resultantly, TPBD also emitted at longer wavelengths up to $624 \mathrm{~nm}$. Another terpyridine-based fluorescent material has been investigated by Song et al., which highlighted the relatively large Stokes shift, quantum yield and strong solvatochromism properties. ${ }^{34}$ Such characteristics were achieved by integrating an acceptor moiety ( $N, N$-diphenylaminophenyl aldehyde) into the terpyridine system, which served as the donor moiety (with electrons coming from nitrogens), thus conferring an efficient charge transfer in the overall molecule. In alcoholic solvents, this derivative emitted at long wavelengths (ca., 505-524 nm), which was detected to be due to intramolecular charge transfer (ICT) in the excited states and the twisting dynamic of the terpyridine unit. Several achievements incorporating terpyridine motifs in white emission include co-polymerization with a polyacrylamide network in a hydroge ${ }^{35}$ and the formation of a supramolecular polymer gel with pyridinium salt units to emit strong white light at $\sim 474 \mathrm{~nm}$ and $571 \mathrm{~nm}^{36}$

One of the highly acclaimed fluorescent dyes is the pyrene chromophore. Due to its ability for strong $\pi-\pi$ interaction, a pyrene monomer can form an excimer (excited state dimer) species. In 2017, a large Stokes shift and bright red-emitting pyrene was developed by introducing a $\pi$-acceptor system. ${ }^{37}$ The pyrene was coupled with a pyridinium moiety to establish a strong push-pull effect with concentrated electrons coming from the highly conjugated pyrene. The compound successfully emitted light at $610 \mathrm{~nm}$ with a Stokes shift of up to $130 \mathrm{~nm}$, making it biologically useful for cell staining. An extension of the acceptor strength was pursued by Chen et al. in 2020, whereby a boronic moiety was engulfed to further extend the acceptor fragment. ${ }^{38}$ Interestingly, a mega Stokes shift of up to $220 \mathrm{~nm}$ was observed as the compound bound to detect hydrogen peroxide, thereby reducing crosstalk between the excitation $\left(\lambda_{\mathrm{ex}}=380 \mathrm{~nm}\right)$ and emission light. Chao and coworkers designed a pyrene-based colorimetric and fluorescent $\mathrm{pH}$ probe with a large Stokes shift of up to $145 \mathrm{~nm} .{ }^{39}$ 1-Acetylpyrene was mixed with 2-aminopyridine-3-carbaldehyde in anhydrous ethanol to afford 2-pyrenyl-1,8-naphthyridine (PNY). PNY initially showed two absorption bands at $271 \mathrm{~nm}$ and $368 \mathrm{~nm}$. Upon acidification, the absorbance at $271 \mathrm{~nm}$ increased, and an opposite trend was observed for the $368 \mathrm{~nm}$ band. In excess acidic condition, new peaks at $322 \mathrm{~nm}, 338 \mathrm{~nm}$ and $429 \mathrm{~nm}$ appeared and gradually increased, which happened concomitantly with a distinct color change from colorless to yellow. The fluorescence wavelength was observed at $515 \mathrm{~nm}$, which prevented self-quenching of the dye. These significant characteristics prompted future study of the pyrene molecules in display, sensing and imaging applications.

Another notable fluorescent dye that is capable of emitting light in the orange to red region $(500-600 \mathrm{~nm})$ of the visible spectrum is the classical 4,4-difluoro-4-bora-3a,4a-diaza-sindacene (BODIPY) based dye. In 2013, novel non-symmetrical coumarin-fused BODIPY dyes were discovered by Bochkov and his group. ${ }^{40}$ This series of dyes (Fig. 3) were reported to show near-infrared (NIR) emission with an exceptionally large Stokes shift and good fluorescence quantum yield. Compound 1a has

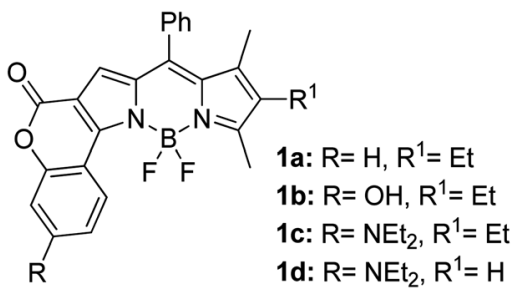

Fig. 3 Diethylamino-substituted dyes show large Stokes shifts in polar solvents (MeOH and DMF). The compounds are from ref. 40. 
a narrow Stokes shift of 30-40 nm in different types of solvents because of the non-substituted coumarin that is fused to BODIPY, while compound $\mathbf{1 b}$ is more promising, having a Stokes shift of almost $60 \mathrm{~nm}$ in DMF. However, when 7diethylamino-4-hydroxycoumarin is fused to BODIPY, a dramatic bathochromic shift of the excitation and emission bands was observed, reaching up to $144 \mathrm{~nm}$ Stokes shift value in a similar solvent. This is the highest ever reported Stokes shift for BODIPY derivatives to the best of our knowledge. This phenomenon can be associated with the strong ICT character of the coumarin and BODIPY in the excited state. Typically, emissive states with ICT feature are weakly fluorescent in polar solvents compared to that in non-polar solvents. This corroborates with results observed in Bochkov et al.'s study, where the fluorescence quantum yields of $\mathbf{1 b}$ were 0.71 and 0.65 in toluene and methanol, respectively. Quenching was detected in a polar aprotic solvent (DMF) with $\Phi_{\mathrm{F}}=0.15$. Meanwhile, a large disparity was detected for 1c when tested in non-polar and polar solvents. The diethylamino-substitution on the coumarin moiety produced $\Phi_{\mathrm{F}}=0.86$ and $\Phi_{\mathrm{F}}=0.62$ in cyclohexane and toluene, respectively, while strong quenching occurred in methanol $\left(\Phi_{\mathrm{F}}=0.06\right)$ and $\operatorname{DMF}\left(\Phi_{\mathrm{F}}=0.04\right)$. It was understood that the formation of twisted ICT (TICT) caused a full charge separation in polar media (a positively charged dialkylamino group rotated perpendicularly to the plane of the molecule). The TICT excited states resulted in a considerably high percentage of the non-radiative decay pathway. The incorporation of BODIPY dyes in stride for white light emission has been recently demonstrated through the supramolecular assembly of three organic components emitting at different wavelength regions. ${ }^{\mathbf{4 1}}$

Many coumarin dyes exhibit large Stokes shifts and fulfill the requirements for use in bio-imaging and optoelectronics. Generally, neutral, compact, and medium polarity compounds (several heteroatoms as hydrogen bond donors and acceptors) are suitable to cross the membranes of living cells. For instance, the mega Stokes shift dyes from Dyomics are coumarins absorbing around 500-520 $\mathrm{nm}$ and emitting in the region of 590-670 $\mathrm{nm}$ in ethanolic solvent. ${ }^{42}$ Emitting at much lower energy (>600 $\mathrm{nm}$ ) proffers less scattering and safer biochemical communication in the human body. There are many studies in the literature presenting large or mega Stokes shifts for coumarin derivatives. Chen et al. reported new pyrrolocoumarin derivatives with remarkably large Stokes shifts. Aiming for coumarin as the parent skeleton, their synthetic route proceeded through Fischer's indole synthesis and Suzuki coupling to form either V-shaped or linear molecules (Fig. 4). ${ }^{43}$ The highlight of their discovery is compound $\mathbf{2 b}$, which was reported to exhibit a Stokes shift of $113 \mathrm{~nm}$ and long emission wavelength at $523 \mathrm{~nm}$, producing an intense green fluorescence $\left(\Phi_{\mathrm{F}}=0.55\right)$. This provides potential applicability of this fluorescent compound in biological FRET devices, with more efficient energy transfer. Compound 2a has a Stokes shift of $159 \mathrm{~nm}$, which is reported to be the highest among the series of derivatives that they synthesized, and is related to the presence of a strong electron withdrawing cyano-group within the molecule. Nevertheless, a large Stokes shift always happens collectively with a non-radiative decay pathway. In their study,<smiles>Cc1[nH]c2ccc3oc(=O)ccc3c2c1C</smiles><smiles>Cc1[nH]c2cc3ccc(=O)oc3cc2c1C</smiles>

V-shaped pyrrolocoumarin Linear pyrrolocoumarin<smiles>Cc1[nH]c2ccc3oc(=O)cc(-c4ccc(C#N)cc4)c3c2c1C</smiles>

2a

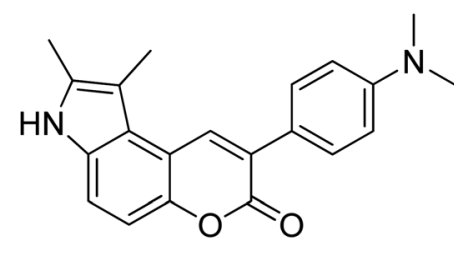

$2 b$
Fig. 4 Pyrrolocoumarin derivatives with chemical modification. The compounds are from ref. 43.

2a exhibits a low fluorescence quantum yield of 0.03. A more detailed exploration on the photophysical nature of coumarin chromophores upon attaching different substituents was evaluated by Liu et al. Apparently, attaching an electronwithdrawing group at the 4-position of coumarin will greatly stabilize the LUMO, and it is thus able to reduce the energy band gap between the HOMO and LUMO. This is due to the higher atomic contribution to the aforementioned position, as compared to the rest of the positions available in a coumarin structure. ${ }^{44}$ The discovery by Liu and co-workers consolidates with the red-shifted emission wavelength observed in $2 \mathbf{a}\left(\lambda_{\mathrm{em}}=\right.$ $547 \mathrm{~nm})$ compared to that in $2 \mathrm{~b}\left(\lambda_{\mathrm{em}}=523 \mathrm{~nm}\right)$. Integrals between coumarin and naphthalimide were reported to nearly construct an ideal white light with a CIE coordinate of $(0.29$, 0.33 ) in the solid form. ${ }^{45}$ Furthermore, tunable fluorescence derived from the host-guest system of perylene bis(diimide) (PDI) and coumarin has been recently reported, successfully covering the whole white light region with a CIE coordinate of $(0.32,0.35)$ upon excitation at $360 \mathrm{~nm}$. Jin and his research group developed a host-induced white emission for the mixture of coumarin and PDI using the macrocycle cucurbit[8]uril to boost the fluorescence intensity and efficiency. ${ }^{46}$ These are clear examples of coumarin derivatives that may be useful in whiteemitting applications.

The screening of large Stokes shift compounds can be particularized when evaluating candidates, especially from physiologically withstanding material like in bioimaging and molecular probe applications. Benzoxazole, benzothiazole and benzimidazole molecules are renowned to be utilized in such applications due to their salient photophysical characteristics, such as dual-emission, ease of synthetic modification and good photostability. ${ }^{47-49}$ Gao et al. synthesized a fluorescent dye, 2,5bis(6-amine-benzoxazol-2-yl)thiophene (BBTA) that possessed strong emission at $568 \mathrm{~nm}$ with the largest Stokes shift of 186 $\mathrm{nm}^{48}$ when tested in buffer solution (phosphate-buffer saline (PBS), $\mathrm{pH}=7.2)$ with $5 \%(\mathrm{v} / \mathrm{v})$ of polyethylene glycol 
monomethyl ether as an additive. This additive increased the aqueous solubility of BBTA and caused the molecules to aggregate in the ground state, which slightly shifted the maximum absorption of the fluorophore towards the UV range at around $382 \mathrm{~nm}$. The result depicts a distinct gap between the maximum absorption and emission wavelength that consequentially gave rise to an exceptionally large Stokes shift. BBTA was also reported to exhibit excellent stability over a wide $\mathrm{pH}$ range and excellent photostability, which makes it a feasible material in lighting applications. Zhao and co-workers developed a ratiometric fluorescence probe with large Stokes shift for the detection of $\mathrm{Cu}^{2+}$ based on a new clamp-on unit. ${ }^{50}$ The molecular design is based on ortho-arylethylnyl benzothiazole, an aromatic alkyne that exhibits a maximum absorption wavelength at $375 \mathrm{~nm}$ and maximum emission wavelength at $566 \mathrm{~nm}$ (Stokes shift of $191 \mathrm{~nm}$ ). The notion of this observation is the presence of a benzothiazole unit that promotes ESIPT within the molecule. Upon coordinating with $\mathrm{Cu}^{2+}$, the emission wavelength blue-shifted to around $446 \mathrm{~nm}$ because of the "push-pull" structure that draws the electron density from the triple bond towards the copper ion. This induced high selectivity of the designed compound towards $\mathrm{Cu}^{2+}$, making it a good indicator as a probe. Recently, Xie and his co-workers synthesized two dual-emissive fluorescent dyes based on the coumarin core and benzothiazole moiety. ${ }^{51}$ Both derivatives (3a and $\mathbf{3 b}$ in Fig. 5) showed a notably large Stokes shift of up to $188 \mathrm{~nm}$ in toluene. The Stokes shift for the derivatives are relatively large compared to most of the coumarin-benzothiazole fluorophores that have ever been reported. The multi-emission properties of these compounds become particularly interesting for white light electroluminescence application because of the ability to circumvent the molecular self-absorption problem that disrupts the white light intensity and color purity. From the Xie group analysis using the time-dependent density functional theory (TDDFT) method, it was found that there is a redistribution of the frontier molecular orbitals at the excited states of the compound after absorbing light. Existing as an enol form in the ground state $\left(\mathrm{S}_{0}\right)$ and keto form in the excited state $\left(\mathrm{S}_{1}\right)$, the molecular orbitals of compound $\mathbf{3 a}$ in the latter state is simulated to be more localized, creating a small energy band gap between its HOMO and LUMO that can rationalize the large Stokes shift event. The difluoroboron bound derivative (3b) produced dual emission even with the absence of the enol rotamer structure, which confirmed that the large Stokes shift can occur upon reallocation of the molecular orbitals.

Another attractive organic scaffold that exhibits a large Stokes shift is the quinoline unit. This privileged structure has been widely used as a core molecular fragment in pharmaceuticals, biocompatible substance, stimulated emission depletion (STED) microscopy, and FRET imaging. ${ }^{52}$ False-positive outcomes from overlapping in the emission and excitation spectra of the donor and acceptor molecule, respectively, can be resolved by integrating a large Stokes shift dye. ${ }^{52,53}$ The provision of such integral would allow scientists to perform robust intermolecular FRET assays to investigate the molecular interactions. Styrylquinolines developed by Czaplińska and coworkers displayed a remarkable push-pull capability. ${ }^{52}$
Derivatives bearing strong electron-donating substituents produced a markedly enhanced extinction coefficient of the low energy band and exceptionally large Stokes shifts in ethanol (131-152 nm). Furthermore, the influence of the bithienyl and $\mathrm{N}$-octyl-carbazyl groups on quinolines has been extensively investigated by the same group, showing distinct photophysical characteristics. ${ }^{31}$ In Czaplińska et al. 's work, it was found that $N$ octyl-carbazole moieties provided a better quantum yield $\left(\Phi_{\mathrm{em}}\right.$ $=9-70 \%)$ and longer lifetime $(\tau=0.83-5.72 \mathrm{~ns})$ as compared to the bithiophene moieties. An enlarged Stokes shift was realized in a quinoline- $N$-octyl-carbazole system that contained a strong electron-withdrawing nitro $\left(\mathrm{NO}_{2}\right)$ group $\left(\lambda_{\mathrm{em}}>600 \mathrm{~nm}\right)$. It was understood that the $\mathrm{NO}_{2}$ facilitates relaxation processes by intensifying the acceptor nature of the quinoline unit. Quinolines are capable of upholding ICT due to its electron-accepting nature from the well-defined dipole moment. Hisham et al. reported a large Stokes shift in the range of 93-120 nm for $N$ aryl-2-aminoquinolines bearing strong electron-donating substituents $\left(\mathrm{CH}_{3}, \mathrm{CH}_{2} \mathrm{CH}_{3}, \mathrm{OCH}_{3}, \mathrm{OH}\right)$ at the para position on the aryl moiety. ${ }^{54}$ This rationalized the delocalization of electrons from the aryl moiety into the quinoline ring, which strongly withdraws electrons. Closely related nitrogen-bearing heteroaromatic frameworks that were studied for their large Stokes shift include derivatives of quinoxaline and pyridine. Interestingly, a T and V-shaped donor-acceptor-donor (D-A-D) structure in the context of a single molecular material has been explored from the combination of these heteroaromatics. In 2018, Sk and his research group integrated a pyridoquinoxaline skeleton as the acceptor core, linked to carbazole groups as the electron-donating groups with the provision that they have excellent electronic properties, high thermal stability, and low cost. ${ }^{55}$ A single-component white light emission was observed, including the temperature-induced tunable emission from blue to orange region of the visible spectrum. In solvents of varying polarity, the compound showed an absorption peak at around $340 \mathrm{~nm}$ with shoulders at 390 and $440 \mathrm{~nm}$. However, the emission spectra were recorded at $\lambda_{\mathrm{em}}=564 \mathrm{~nm}$ (yellow fluorescence) in toluene, which gradually red-shifted to $\lambda_{\mathrm{em}}=$ $631 \mathrm{~nm}$ (red fluorescence) in the more polar dichloromethane (refer Fig. 6). This gives rise to the solvent-dependent high

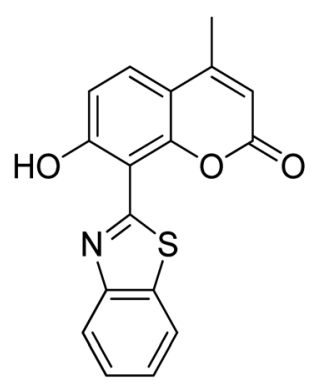

$3 a$

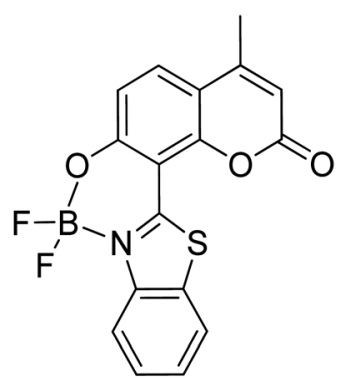

$3 b$
Fig. 5 Dual-emissive coumarin-based dyes with 8-benzothiazole (left) and its difluoroboron bound derivative (right). The compounds are from ref. 51 
(a)

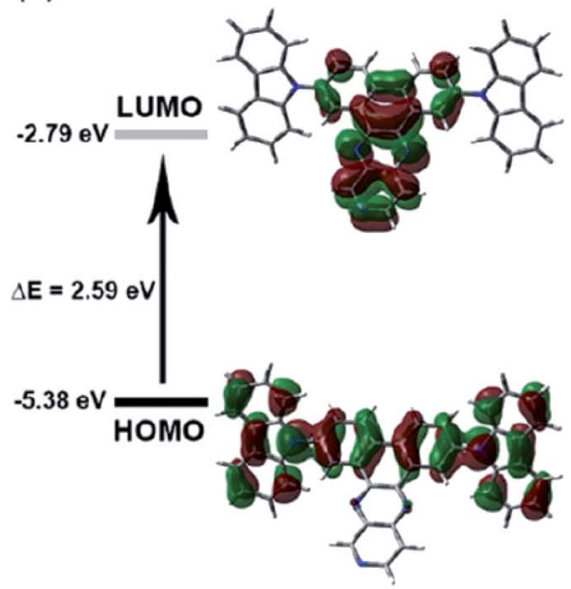

(a)

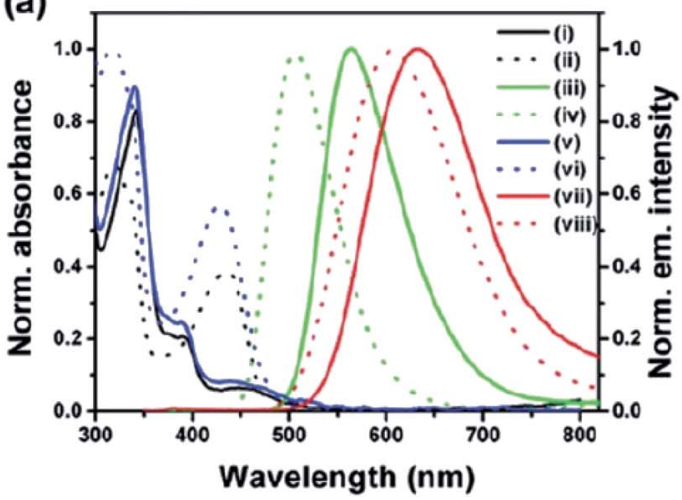

(b)

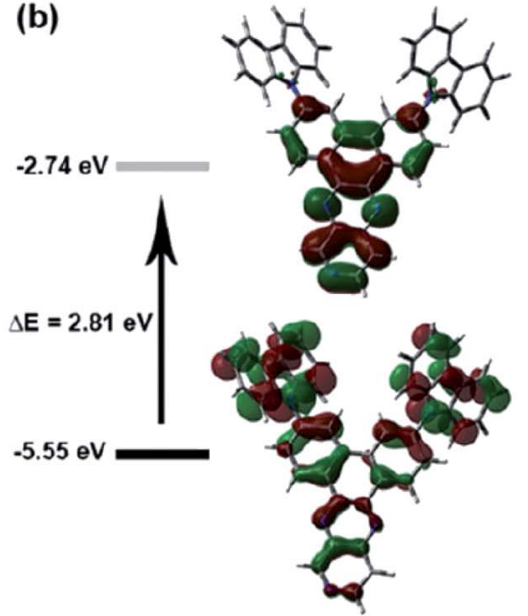

(b)

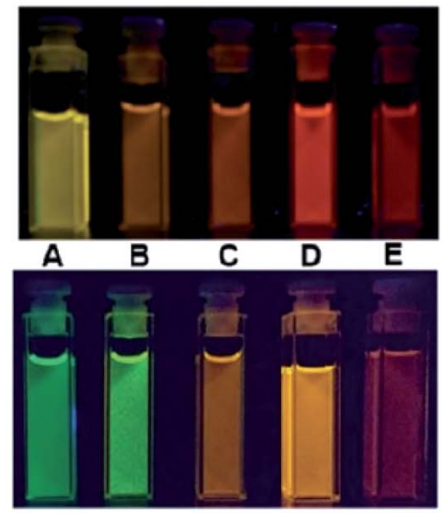

Fig. 6 (Top) The optimized ground-state HOMO and LUMO distributions, and the respective energy values of (a) PQCz-T and (b) PQCz-V obtained by DFT calculations at the B3LYP/6-31G(d,p) level. Bottom: (a) normalized absorption and emission $\left(\lambda_{\text {ex }}=340 \mathrm{~nm}\right)$ spectra of PQCz-T (solid line) and PQCz-V (dotted line) in toluene (absorption: black), emission: green, (i-iv) and $\mathrm{CH}_{2} \mathrm{Cl}_{2}$ (absorption: blue), emission: red, (v-viii). (b) The digital photographs of PQCz-T (upper panel) and PQCz-V (bottom panel) in solvents of varying polarity under the illumination of a UV lamp $\left(\lambda_{\text {ex }}=365 \mathrm{~nm}\right.$ ). (A) Toluene (0.099), (B) 1,4-dioxane (0.164), (C) tetrahydrofuran (0.207), (D) chloroform (0.259) and (E) dichloromethane (0.309). The solvent polarity parameter ( $E_{\mathrm{T}}^{\mathrm{N}}$ value) is mentioned in the parenthesis. The figure was reproduced from ref. 55 with copyright permission from The Royal Society of Chemistry.

Stokes shift of the compound, which agrees with the facile ICT and possible twisted ICT (TICT) state due to the effortless D-A rotation. $\mathrm{Xu}$ et al. reported surprisingly high external quantum efficiencies (EQE) for bicyclic fused pyrazine of up to $7.37 \%$, which justifies that the nitrogen-bearing main chromophores could potentially be applied in optoelectronic and light-emitting diode applications in the future. ${ }^{56}$ The theoretical limit EQE for the fluorescent emitter is only 5\%, thereby opening versatile avenues of nitrogen-fused aromatics.

Continuous effort in searching for a low-cost emitter with a large Stokes shift was realized by Volpi et al. when synthesizing a series of 1,3-diarylated imidazo[1,5-a]pyridine<smiles>COc1ccccc1-c1nc(-c2ccccc2)c2ccccn12</smiles>

$4 a$<smiles>c1ccc(-c2nc(-c3ccccc3)n3ccccc23)cc1</smiles>

4b<smiles>c1ccc(-c2nc(-c3cccc4ccccc34)n3ccccc23)cc1</smiles>

$4 c$<smiles>c1ccc(-c2nc(-c3c4ccccc4cc4ccccc34)n3ccccc23)cc1</smiles>

4d

Fig. 7 Different substituents in position 3 of the 1-phenylimidazo[1,5-a]pyridine skeleton. The compounds are from ref. 57. 
derivatives. ${ }^{57}$ We selected several of their compounds (Fig. 7) to discuss the absorption and emission properties. Compound $4 \mathbf{4 a}$ has a Stokes shift of $174 \mathrm{~nm}$ and high fluorescence quantum yield, $\Phi_{\mathrm{F}}=0.385$, as compared to the rest of the molecules. Thus, it was selected to test as a luminescent low-cost material candidate for downshifting application. The compound was uniformly dissolved in a transparent thermosetting polyurethane resin in a $1: 1$ ratio to produce solid fluorescence. The absorption $\mathbf{4 a}$ in the solid state and in dichloromethane solution showed no changes, while the emission in the solid state was blue-shifted by about $15 \mathrm{~nm}$ as compared to the solution. From compound $\mathbf{4 b}$ to $\mathbf{4 d}$, the extension of the conjugation in position 3 showed bathochromic shift emission wavelengths in the region of $460 \mathrm{~nm}-550 \mathrm{~nm}$ (cyan color). The presence of an anthracene moiety in $\mathbf{4 d}$ has shown a quite large Stokes shift value of $166 \mathrm{~nm}$. However, to a slight dismay, a lower yield was observed with $\Phi_{\mathrm{F}}=0.064$.

A low molecular weight material with a single aromatic ring has been highlighted in a study conducted by Percino and his group. ${ }^{58}$ They introduced two electron-withdrawing cyano groups in 4-((2-hydroxyethyl)(methyl)-amino)benzaldehyde, and compared its luminescence property with the commercial polymer poly(2-methoxy-5(2'-ethyl)hexoxy phenylenevinylene) (MEH-PPV). Compellingly, their compound showed red emission, which red-shifted slightly by $67 \mathrm{~nm}$ when compared to MEH-PPV. Both molecules possessed notable capacity to form a thin film with smooth morphology, thereby making it high likely useful as an OLED. In methanol, the compound 2-(4-((2hydroxyethyl) (methyl)amino)benzylidene)malononitrile produced a large Stokes shift of more than $150 \mathrm{~nm}$, which was due to intra- and intermolecular interactions with solvent molecules. Another work showing a potential organic molecule as a light-emitting diode includes the designation of $5,5^{\prime}-([1,2,5]$ thiadiazolo[3,4-c]pyridine-4,7-diyl)bis( $N, N$-diphenylthiophen-2amine) (DTPS-PT), which generated real NIR emission with $\lambda_{\text {onset }}>700 \mathrm{~nm}$ in both doped and nondoped devices. ${ }^{59}$ Gratifyingly, the molecule was able to achieve a maximum radiance of $2202 \mathrm{~mW} \mathrm{Sr}^{-1} \mathrm{~m}^{-2}$, which is known to be one of the highest values ever reported for NIR-OLEDs. The central pyridine thiadiazole (PT) worked as an acceptor against the donor diphenylamine thiophene (TPS). These examples depicted a wide selection of organic molecules that exhibit large or mega Stokes shift property and outstanding luminescence responses, which make them highly sought after as low-cost alternatives to the existing metal-based or inorganic emitters. ${ }^{60}$ Nevertheless, a deeper understanding of the processes for different chromophores is crucial in order to modify the current molecular structure to achieve better optical features. The details of these mechanisms will be discussed in Sections 3.1-3.7.

\subsection{Inorganic molecules}

As for inorganic-based materials, large Stokes shift molecules can be derived from a group of metal complexes, organicinorganic hybrid scaffolds, carbon dots and quantum dots (QDs). In 2015, Li et al. recently developed a one-pot method of synthesizing colloidal $\mathrm{CuInS}_{2} / \mathrm{ZnS}$ core/shell quantum dots using the air-stable non-coordinating solvent paraffin liquid to reduce the reaction rate, obtaining spherical shape and monodispersed QDs. ${ }^{61}$ When tested in chloroform, the fluorescence peak was recorded at $550 \mathrm{~nm}$ and the full width at half maximum (FWHM) was detected at $125 \mathrm{~nm}$. $\mathrm{CuInS}_{2} / \mathrm{ZnS}$ also has a noteworthy photoluminescence quantum yield that reached up to $81 \%$. In addition, it displayed a large Stokes shift that reached more than $150 \mathrm{~nm}$, thereby making it suitable as a luminescent solar concentrator (LSC). The $\mathrm{CuInS}_{2}$ core and $\mathrm{Zn}-\mathrm{CuInS}_{2}$ nanocrystals dominated the emission wavelength, while the ZnS shell with a wider band gap dominated the absorption wavelength. The typical binary QDs' inorganic coating mechanism (Fig. 8) explains the nature of wider band gap materials, like $\mathrm{ZnS}$, that act as a shell by epitaxial growth on cores with a lower valence band and higher conduction band than that of the core materials to acquire the core-shell structures. On the basis of this mechanism, the inorganic shell coating has a low self-absorption (large Stokes shift) and high quantum yield. Nowadays, the fabrication of eco-friendly carbon quantum dots is attainable by scientists. It was proven by Zhao and co-workers from their synthesis using the spaceconfined vacuum heating approach with citric acid or urea as precursors and water as the solvent. ${ }^{62}$ In Zhao's study, the large Stokes shift of carbon dots was required for large-scale LSC application, and aimed for the removal of light losses due to reabsorption during light collection. Hence, they designed colloidal carbon dots with a quantum yield of $\sim 65 \%$ and Stokes shift value of $0.53 \mathrm{eV}$. The proceeded synthetic method was reported to achieve 50\% larger Stokes shift than in conventional solvothermal method (Stokes shift of $0.36 \mathrm{eV}$ ). The large Stokes shift can be attributed to a recombination of the electron-hole pair with a single type of dominant energy state that differed
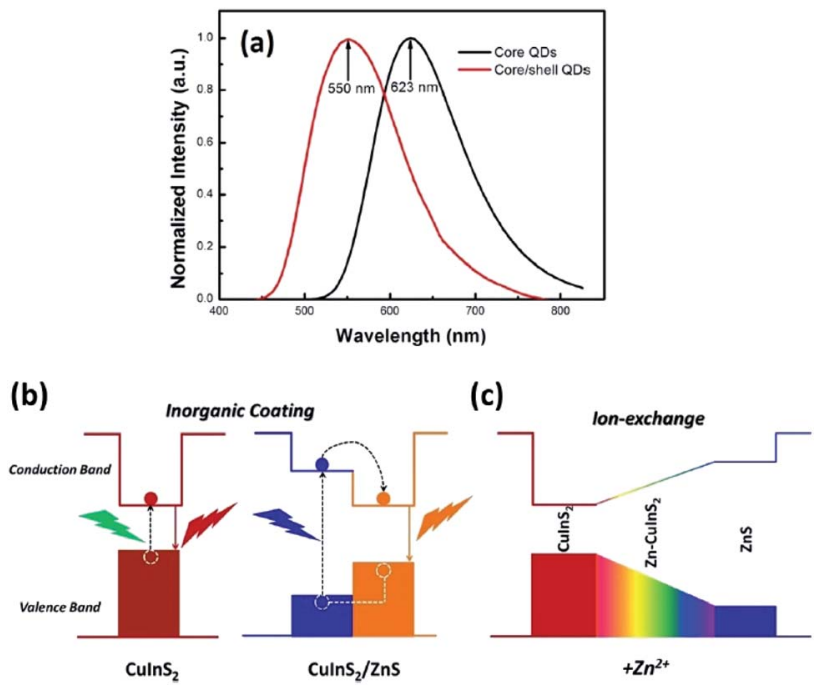

Fig. 8 (a) Normalized emission spectra of the CulnS $_{2}$ core QDs and CulnS $2 / Z n S$ core/shell QDs. (b) Photoluminescence emission mechanism of the $\mathrm{CulnS}_{2} / \mathrm{ZnS}$ core/shell QDs. (c) Schematic of the changes of the energy band gap with the addition of $\mathrm{Zn}^{2+}$. The figure was reproduced from ref. 61 with accreditation given to Creative Commons Attribution 4.0 International Public License. 
(a)

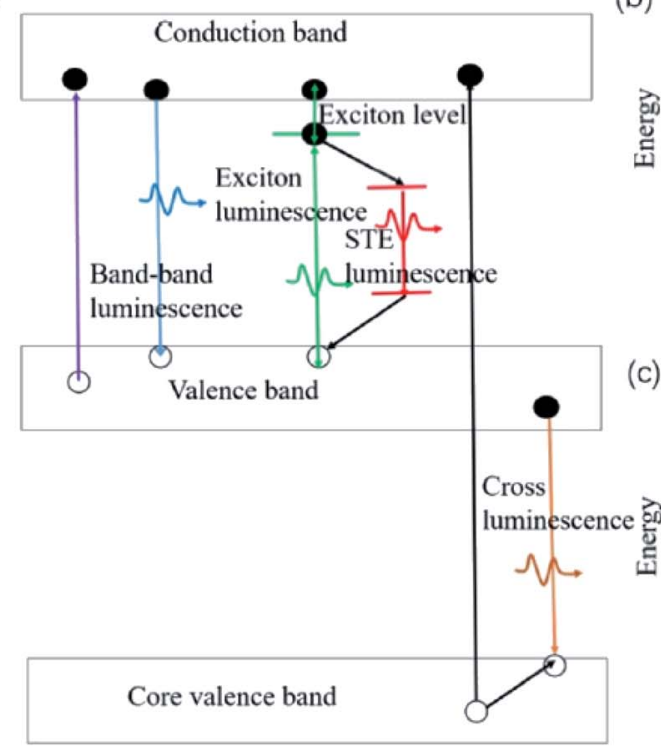

(b)

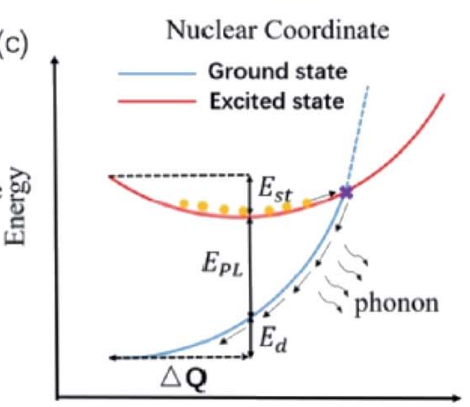

Nuclear Coordinate

Fig. 9 (a) Schematic of various intrinsic photoluminescence phenomena, including band-to-band luminescence, exciton luminescence, STE luminescence, and cross luminescence. (b) Schematic of the energy level structure of STE (GS, ground state; FE, free exciton state; FC, free carrier state; STE, self-trapped exciton state; $E_{\mathrm{g}}$, band gap energy; $E_{\mathrm{b}}$, exciton binding energy; $E_{\mathrm{st}}$, self-trapping energy; $E_{\mathrm{d}}$, lattice deformation energy; $E_{\mathrm{PL}}$, emission energy). (c) Schematic of the non-radiative recombination process for STE. Orange circles represent the excited electrons. The figure was reproduced from ref. 69 with permission. Copyright 2019 American Chemical Society.

from the band-gap emission. Also associated with solar cell technology, Khan et al. synthesized N-doped graphene quantum dots with an ultra-high photoluminescence quantum yield (99\%) and large Stokes shift $(98 \mathrm{~nm})$ as downconverters in copper indium gallium selenide (CIGS) solar cells. ${ }^{63}$ Inorganic quantum dots are nano-sized semiconducting crystals, which provide robust tunability of the absorption and emission bands. Through the thermal treatment of graphene oxide and polyethylenimine composite, the doping concentration of the nitrogen and oxygen moieties can be controlled to achieve a desirable photoluminescence quantum yield and Stokes shift value. The conversion efficiency of Khan's solar cell increased up to $15.31 \%$ via downconversion and light -trapping effect.

The endeavor to explore large Stokes shift inorganic molecules was also realized by Shishino and his group, which pivoted to luminescence in gold nanoparticles (AuNPs). ${ }^{64}$ Even with diameters smaller than $2 \mathrm{~nm}$, this material is luminescent, making them an attractive alternative to quantum dots. The large Stokes shift of AuNPs was ascribed to the luminescence from a low-lying triplet state populated by intersystem crossing (ISC) from the lowest singlet state. Produced by molten matrix sputtering (MMS) method, their AuNPs depicted a large Stokes shift value due to the absorption peak at wavelengths shorter than that of previously reported AuNPs. Since MMS involves a temperature-controlled dispersion medium during the sputtering deposition, the large Stokes shift may correspond to the stabilization of the $\mathrm{d}$ band and/or destabilization of the spconduction band of the AuNPs. Wen and co-workers also worked on fluorescent Au nanoclusters (AuNCs) with a mega Stokes shift of up to $237 \mathrm{~nm}$ that can detect berberine hydrochloride (BHC) ${ }^{65}$ The AuNCs were prepared from citratestabilized stannous chloride and hydrogen tetrachloroaurate(III) as raw materials via hydrothermal method. The material was capable of fluorescing at long wavelength emission $(566 \mathrm{~nm})$ and quenched gradually upon increasing detection of BHC, which made it remarkably useful as a sensitive and selective spectrofluorometric probe.

Metal complexes are capable of undergoing ultrafast excited state structural deformation. In this context, Zhou and coworkers designed an organic seesaw-shaped tin bromide with deep-red emission $\left(\left(\mathrm{C}_{9} \mathrm{NH}_{20}\right)_{2} \mathrm{SnBr}_{4}\right) \cdot{ }^{66}$ This organic metal halide hybrid possessed a zero-dimensional (OD) structure, allowing bulk crystals to exhibit intrinsic properties of individual $\mathrm{SnBr}_{4}{ }^{2-}$ species in solid state. Decreased dimensionality leads to the emergence of unique properties like exciton selftrapping or excited state structural deformation. Upon photoexcitation, these bulk crystals produced a highly efficient broadband deep-red emission peaked at $695 \mathrm{~nm}$, which has a conspicuous Stokes shift value of $332 \mathrm{~nm}$. The bulk crystals also showed an appreciably high quantum efficiency ( $\sim 46 \%)$ and long single exponential lifetime $(6.51 \mu \mathrm{s})$, which is consistent with the characteristics of a phosphorescent molecule. The seesaw structure enabled a pronounced excited state structural deformation as confirmed by DFT calculations, which was responsible for a large Stoke shift. Platinum complex motifs with the mega Stokes shift have been explored by Liu et al. by connecting a quinolinium moiety (A) and a triphenylamine derivative moiety (D) with a carbon-carbon double bond. ${ }^{67}$ The tripodal structure with triphenylamine at the centre linked its remaining two arms with pyridine coordinated to Pt moieties. 
The overall Pt complex showed two absorption bands at approximately $400 \mathrm{~nm}$ and $490 \mathrm{~nm}$ in acetonitrile solution. The low energy band was tentatively assigned to an intraligand charge transfer (ILCT) transition from triphenylamine to the quinolinium moieties. Emissions were observed at around $530 \mathrm{~nm}$ and $700 \mathrm{~nm}$, which originated from a triplet metalperturbed intraligand $\left({ }^{3} \mathrm{IL}\right)$ excited state and charge transfer excited state character, respectively. Based on TDDFT study, it was confirmed that the spin density was predominantly localized on the organic moieties of the Pt complex, which further supported the ${ }^{3}$ ILCT character for the low energy emission band ( $\sim 700 \mathrm{~nm}$ ). The enhanced coplanarity of the phenyl ring with the quinolinium moiety in the $\mathrm{T}_{1}$ geometry and increased dipole moment from the $S_{0}$ to $T_{1}$ states are believed to contribute to the mega Stokes shift property of the complex. The complex was further tested by being stained on HeLa cells prior to analysis with super-resolution microscopy based on structured illumination microscopy (SIM). The results showed an improvement in the signal-to-noise ratio and good photobleaching resistance.

Zhang and co-workers demonstrated inorganic lead halide perovskite nanocrystals (NCs), which are built on the trigonal $\mathrm{Cs}_{4} \mathrm{PbX}_{6}(\mathrm{X}=\mathrm{Cl}, \mathrm{Br}, \mathrm{I}) .{ }^{68} \mathrm{Cs}_{4} \mathrm{PbCl}_{6}$ NCs with sizes of $2.2-11.8 \mathrm{~nm}$ were synthesized by using the solvothermal method. This type of crystal structure is said to have a wider band gap and distinct optoelectronic properties compared to its cubic counterpart, $\mathrm{CsPbX}_{3}$. It has shown a considerably large Stokes shift of $75 \mathrm{~nm}$ for the inorganic NCs structure, which can be associated with the lattice relaxation. A more detailed discussion of this type of crystal revealed that the $\mathrm{Cs}_{4} \mathrm{PbX}_{6}$ crystal possesses the Frenkeltype excitons that are trapped by the $\mathrm{PbX}_{6}{ }^{4-}$ octahedrons. Due to its indirect band gap, this crystal structure has a relatively strong luminescence coming from the self-trapped excitons
(STE) at low temperature. $\mathrm{Cs}_{4} \mathrm{PbCl}_{6}$ emitted a single smooth near-UV band that was gradually red-shifted, and peaked at $360 \mathrm{~nm}$ as the excitation wavelength increased. The quantum confinement effect accounts for the shifting of the emission wavelength, giving rise to a significant Stokes shift for such small sample. The STE has thus become a useful indicator to assess for large Stokes shift characteristics in all-inorganic halide perovskites. Li et al. reviewed several perovskites with STE emission, which were promising for solid-state lighting. ${ }^{69}$ Before delving into the examples of halide perovskites, they outlined the phenomenon of intrinsic luminescence as in Fig. 9. STE has an emission energy that is much smaller than the band gap of the exciton binding energy, and thus generally features a broad spectrum. Materials like $\mathrm{Cs}_{3} \mathrm{Cu}_{2} \mathrm{I}$ and $\mathrm{Cs}_{2} \mathrm{Na}_{x} \mathrm{Ag}_{1-x} \mathrm{InCl}_{6}$ are said to be good STE emitters due to their capability to suppress the self-absorption, leading to a large Stokes shift. They are capable of generating a large electron-phonon coupling and soft lattice. A mixture of $\mathrm{Cs}_{3} \mathrm{Cu}_{2} \mathrm{I}$ with a yellow phosphor can induce a white phosphor, while the optimized $\mathrm{Cs}_{2} \mathrm{Na}_{x} \mathrm{Ag}_{1-x} \mathrm{InCl}_{6}$ has demonstrated a warm white lightemitting diode. Another important characteristic of the optimized $\mathrm{Cs}_{2} \mathrm{Na}_{x} \mathrm{Ag}_{1-x} \mathrm{InCl}_{6} \mathrm{NCs}$ is that their maximum absorption wavelengths are bound to be in the range of the UV region, thus providing the full potential of the excitons to become more transient within the electronic confinements. For instance, $\mathrm{Cs}_{2} \mathrm{AgInCl}_{6}$ has been shown to reveal an absorption peak at exactly $365 \mathrm{~nm}$ upon Na alloying to depress the non-radiative defects, providing a rewarding potential for a high-quality white emission.

White light-emitting diodes comprise a wide color gamut, and are determined by the color coordinates of RGB. Research efforts on the luminescent properties based on green and red emitters have been continuously pursued to gain a narrower
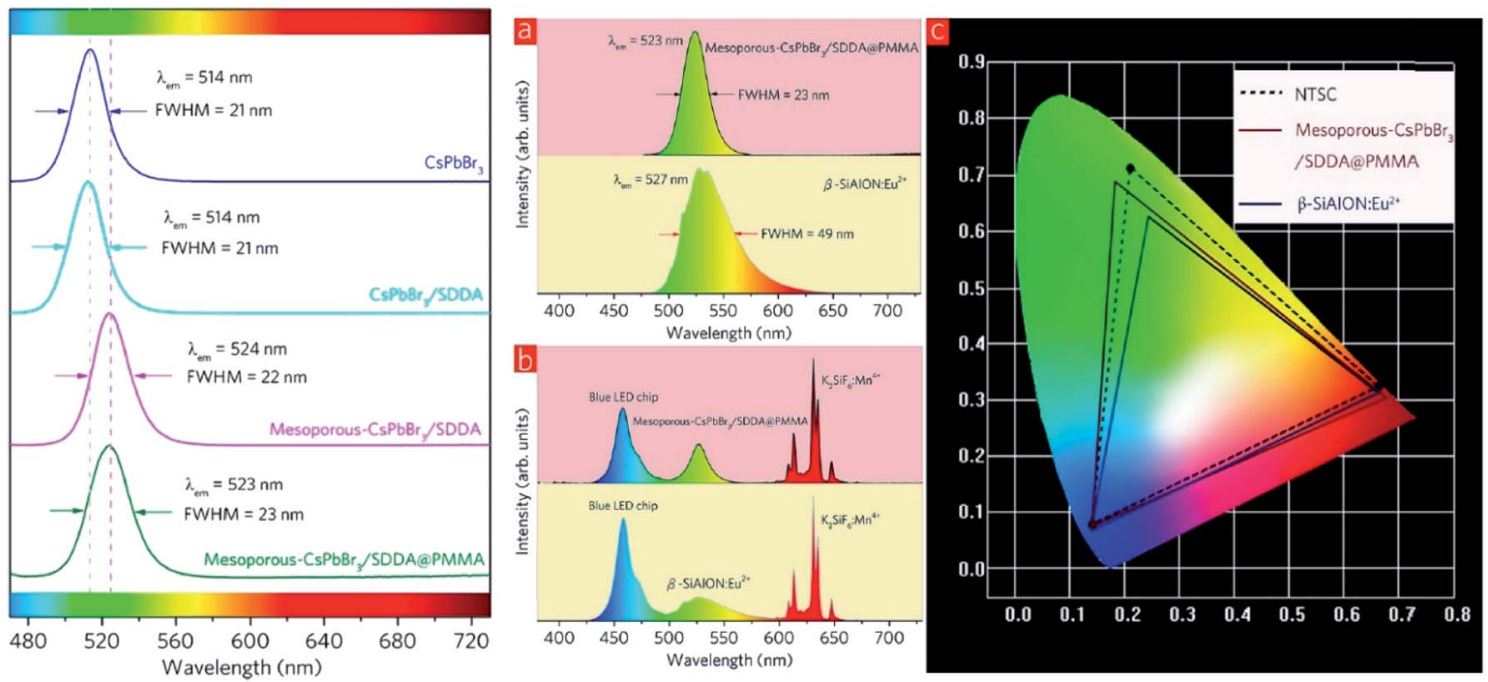

Fig. 10 (Left) $\mathrm{PL}$ spectra of $\mathrm{CsPbBr}_{3}, \mathrm{CsPbBr}_{3} / \mathrm{SDDA}$, mesoporous-CsPbBr 3 /SDDA, and mesoporous-CsPbBr 3 /SDDA(aPMMA. (Right) (a) PL spectra of $\beta$-SiAION : Eu ${ }^{2+}$ phosphor and mesoporous-CsPbBr 3 /SDDA@PMMA powder. (b) EL spectra of two white LEDs employing a $\beta$-SiAION : $\mathrm{Eu}^{2+}$ phosphor and mesoporous-CsPbBr $3 /$ SDDA@PMMA powder. (c) Color gamut of two white LEDs employing a $\beta-S i A l O N$ : Eu ${ }^{2+}$ phosphor and mesoporous-CsPbBr 3 /SDDA@PMMA powder, and NTSC. The figure was reproduced from ref. 70 with permission. Copyright 2016 American Chemical Society. 
color band that would contribute impressively to the white light. Among the inorganic green emitters designed, perovskite $\mathrm{CsPbBr}_{3}$ quantum dots have been particularly interesting and promising due to their high photoluminescence quantum yield. Nevertheless, there are several recognized issues, including the still insufficient efficiencies due to the surface trap states, low thermal stability, and low tolerance to humidity. For these reasons, Zhang and co-workers proposed an inorganic-organic hybrid pair (didodecyl dimethylammonium sulfide, $\mathrm{S}^{2-}-\mathrm{DDA}^{+}$) to passivate the surface defects of the current quantum dots. ${ }^{70}$ Moreover, they suggested a mixture of the $\mathrm{CsPbBr}_{3}$ quantum dots with mesoporous silica particles to overcome the thermal instability, and the subsequent blending of the mesoporous$\mathrm{CsPbBr}_{3} / \mathrm{SDDA}$ with polymethylmethacrylate (PMMA) to diminish the sensitivity of this green emitter towards moisture. White LEDs were fabricated as a proof-of-concept, and it was found that the color gamut achieved up to $102 \%$. Fig. 10 shows the red-shifted emission from the new designation of the inorganic green emitter, which substantially contributed to white light application upon the mixture built-in with the blue LED chip and red emitter of $\mathrm{K}_{2} \mathrm{SiF}_{6}: \mathrm{Mn}^{4+}$. It was noteworthy that even a slight enlargement of the Stokes shift value could significantly render an approaching ideal white light emission and color gamut.

\section{Molecular design for large (mega) Stokes shift material}

The facile mixing of multiple emitters can cause unfavorable interactions between molecules, making it difficult for chemists to design the appropriate molecular structure, be it for organic or inorganic materials. Currently, much effort has been devoted to proposing materials that exploit the concept of ESIPT, ICT, excited state structure deformation, hydrogen bonding mediated $\mathrm{J}$ - or $\mathrm{H}$ - aggregation, aggregation induced emission (AIE), interchange between monomer and excimer fluorescence, host- guest interaction, metal-ligand charge transfer (MLCT), among others.

With this in mind, the photophysical processes will be highlighted and discussed by taking various examples from previously reported materials. The discussion will also cover both individual materials, which can single-handedly produce white light emission, and combinatorial materials being integrated together as a part of the white light system.

\subsection{Excited state intramolecular proton transfer (ESIPT)}

The ESIPT reaction usually incorporates the transfer of a proton donor (e.g., hydroxy or amino proton) to proton acceptor moieties, like carbonyl oxygen or pyridyl nitrogen, in the proximity of a molecule. ${ }^{71}$ Some remarkable properties of ESIPT are the large Stokes shift, ${ }^{72,73}$ dual emission, ${ }^{74}$ ultrafast process, ${ }^{12}$ and the spectral sensitivity to nanoenvironments. ${ }^{73}$ The most widely investigated ESIPT fluorophores revolve around the conventional proton donor or acceptor molecules, such as in 2(2'-hydroxyphenyl)benzimidazole (HBI), benzoxazole (HBO) and benzothiazole (HBT) derivatives. ${ }^{75-77}$

In this perspective, the basic photophysics of ESIPT chromophores have been discussed by Zhao et al., which focuses on the state-of-the-art development of new luminescent materials. ${ }^{12}$ It should be mentioned that chemically modifying the conventional fluorophore to attain an increased Stokes shift can be rigorous. Fig. 11 illustrates the basic photophysical process of ESIPT in a 2-(2'-hydroxyphenyl)benzothiazole (HBT) compound. An intramolecular hydrogen bond between nitrogen and hydrogen atoms is formed at the ground state, giving rise to the cis-enol form. When irradiating photons, the excited state is populated with the enol form, followed by an ultrafast ESIPT process that subsequently produces the cis-keto form at the singlet excited state. The keto tautomer usually accounts for the fluorescence observed in the ESIPT chromophore. There is also a deactivation channel (ISC) of the cis-keto form, which leads to a low-lying triplet excited state.
$E^{*}$
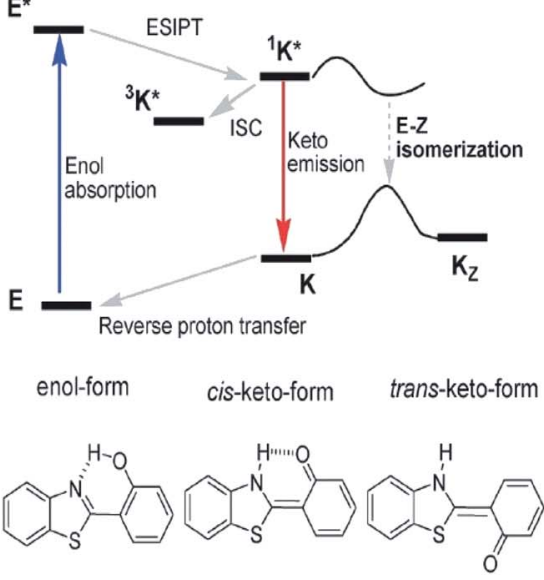
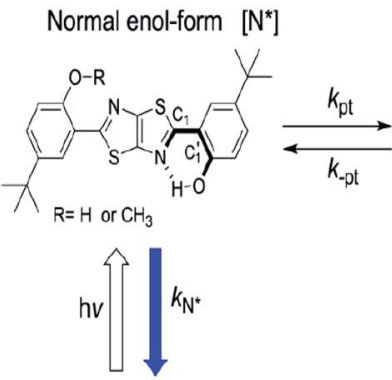

Tautomer keto-form $\left[\mathrm{T}^{*}\right]$

${ }^{a}\left[\mathrm{~N}^{*}\right]$, concentration of normal species of excited state; $\left[\mathrm{T}^{*}\right]$, concentration of tautomer species of excited state; $k_{\mathrm{pt}}$ proton transfer rate constant; $k_{-p t}$ reverse proton-transfer rate constant; $k_{\mathrm{N}^{*}}$ decay rate constant of $\mathrm{N}^{*}$ for all decay channels except $k_{\mathrm{pt}} ; k_{\mathrm{T}^{*}}$, decay rate constant of $\mathrm{T}^{*}$ for all decay channels except $k_{-\mathrm{pt}}$.

Fig. 11 (Left) Principal photophysics of ESIPT. Illustrated by 2-(2'-hydroxyphenyl)benzothiazole. The figure was adapted from ref. 12 with copyright permission from the PCCP Owner Societies. (Right) Reversible ESIPT reaction pattern for two thiazolo[5,4-d]thiazole derivatives. The figures were adapted from ref. 78 with permission. Copyright 2016 American Chemical Society. 
Furthermore, a trans-keto structure may also form via the isomerization non-radiative decay. The decay of these two events, i.e., triplet excited state keto tautomer and trans-keto to cis-keto transformation occurs at the $\mu$ s timescale, which can be considered as slow processes. Both processes can be distinguishable by time-resolved spectroscopy, whereby the decay of the triplet excited state is sensitive to $\mathrm{O}_{2}$, whereas the transformation of the trans-keto to cis-keto form is pretty much unaffected by $\mathrm{O}_{2}$.

Also revolving around the ESIPT system, Zhang et al. reported thiazolo $[5,4-d]$ thiazole $(\mathrm{TzTz})$ derivatives that involved a proton (or hydrogen atom) transfer from a pre-existing hydrogen bond, which has led to a proton-transfer tautomer in the excited state. ${ }^{78}$ In theory, the reaction dynamics of the unimolecular ESIPT system can be harnessed via chemical modification or external factors in a way that the fluorescence is coming from both reactant and product. This provides the possibility to fine-tune the overall luminescence properties of the single compound to generate white light. The strategy to establish the ESIPT system in their study was to integrate two phenol-substituted groups onto the TzTz proton acceptor core, as in Fig. 11. All TzTz molecular structures were confirmed to be planar based on the X-ray diffraction analyses. There was no substantial $\pi-\pi$ stacking because of two very bulky tert-butyl groups. This bulkiness was inserted to increase the solubility of the molecule, which is a key factor for the later femtosecond fluorescence up-conversion study and substantial reduction of any intermolecular contact (solvent effect). A large Stokesshifted yellow emission emerged around $560 \mathrm{~nm}$ for derivatives of TzTz. This emission peak has been assigned to the proton-transfer tautomer emission originating from ESIPT. Fast equilibrium between the two species, $\mathrm{N}^{*}$ and $\mathrm{T}^{*}$, prior to their respective emission contributed to identical population decay time constants between the two. The emission intensity ratios of the TzTz derivatives were sensitive to changes in the solvent polarity. On passing from non-polar (cyclohexane) to polar aprotic (dichloromethane), it was found that the tautomer emission predominated and led to a smaller quantum yield. The mechanism of ESIPT worked in an uneven electron density distribution, arising from enol-keto tautomerization on only one side of the symmetric molecule. This led to an enlargement in the dipole moment in $\mathrm{T}^{*}$, which stabilized further as the solvent polarity increased.

Yadav and co-workers also explored the ESIPT properties of rhodol derivatives that were linked to benzimidazole and benzothiazole units. ${ }^{79}$ Rhodol is generally a hybrid form of fluorescein and rhodamine, which is well-known for its good solubility, photostability and high fluorescence quantum yield. Interestingly, the rhodol derivatives that they designed displayed large Stokes shifts (50-260 nm) in spirocyclic forms, which were assigned to the ESIPT process. The benzimidazole and benzothiazole units were strategically introduced adjacent to the oxygen atom of the rhodol core fragment in expectation that the ESIPT process is prompted between the $\mathrm{OH}$ (rhodol) and $\mathrm{N}$ atom (benzimidazole/benzothiazole). Spirocyclic-rhodols emitted at longer wavelengths, ca. 428-525 $\mathrm{nm}$, than their opened counterparts. An additional privilege of this structural framework is that it possessed strong solvatochromic properties when tested from non-polar to polar solvents. Such phenomenon could be explained by dipole-dipole interaction and solute-solvent interaction, which was proven by Lippert-Mataga and Mac-Rae functions. These advantages provide an avenue for applications in photochromic and laser dyes, and fluorescence recording techniques. Barman et al., on the other hand, designed a drug delivery system (DDS) that exploited the property of ESIPT.$^{80} \mathrm{~A}$ benzothiazole unit was attached to the 8position of 7-hydroxycoumarin before conjugating with the anticancer drug, chlorambucil (Cou-Benz-Cbl). The interconnection between the three organic units successfully produced a large Stokes shift up to $151 \mathrm{~nm}$, which can be ascribed to ESIPT between $\mathrm{OH}$ (coumarin) and $\mathrm{N}$ atom (benzothiazole). In polar protic (ethanol and methanol) and polar aprotic (acetonitrile and tetrahydrofuran) solvents, the compound emitted dual emission at $406 \mathrm{~nm}$ and $516 \mathrm{~nm}$, respectively. The lower energy emission can be attributed to keto formation in the excited state upon photoirradiation. Barma also found that upon strengthening of the hydrogen bonding in a binary mixture of methanol:water, the keto emission intensity became more pronounced.

The $\mathrm{N}-\mathrm{H}$ type ESIPT emitter has an advantage over the $\mathrm{O}-\mathrm{H}$ type because of the three valences of the nitrogen atom. One of them can be used to tailor the molecular structure to control the proton migrating ability and tune the electronic properties. Tseng and co-workers designed a facile, single site amino derivatization in 10 -aminobenzo[ $h]$ quinoline by replacing one of the $\mathrm{N}-\mathrm{H}$ hydrogen atoms with varying substituents to construct a series of excited state intramolecular $\mathrm{N}-\mathrm{H}$ transfer molecules (Fig. 12). ${ }^{81}$ When one of the amino protons was substituted by a strong electron-withdrawing group, it would increase the $\mathrm{N}-\mathrm{H}$ acidity, which promoted the ESIPT process. The general comparison between the presence of these two opposing behaviors within the parent compound is revealed when EWG groups (5e-5i) were shown to emit light in more redshifted regions (590 nm-740 nm) than EDG groups (5a-5d). The compound that has the stronger intramolecular hydrogen bonding strength will have a larger downfield shift of the $\mathrm{N}-\mathrm{H}$ proton and better acidity character. Hence, a faster and highly exergonic type of ESIPT was achieved with increased proton donating strength. This can be rationalized by varying the EWG on the amino site, which results in lowering the HOMO energy and increasing the energy gap of the imino-tautomer emission.

Another ESIPT-based fluorescence was demonstrated by Okamoto and group through the solid-state emission of 3amidophthalimides. ${ }^{82}$ All derivatives (Fig. 13) showed an absorption band in the range of 323-345 $\mathrm{nm}$. Compound $6 \mathbf{a}$ produced red fluorescence at a wavelength of $625 \mathrm{~nm}$ with a very large Stokes shift of $13400 \mathrm{~cm}^{-1}$ when tested in toluene. Similarly, for the remaining derivatives, this behavior can be resolved by the ESIPT phenomenon through their respective tautomer formation upon excitation of the molecules. Compound 6d showed dual emission at $391 \mathrm{~nm}$ and $565 \mathrm{~nm}$, appearing yellow when tested in the solvent toluene. The fluorescence at $565 \mathrm{~nm}$ accounts for the ESIPT process due to the fast equilibrium between the relaxed amide and its tautomer 
species. The aim in modifying the molecular structure for a large Stokes shift property has thus been rectified by tuning the enol and keto emission bands through the ESIPT process. There are two forms of which researchers can opt to approach for obtaining ESIPT-based fluorescence, i.e., tuning molecules in solution or in solid states. Therefore, the fluorescence intensity of molecules can be further optimized so that an appreciable quantum yield can be achieved for the development of white light emission. This can be carried out by introducing different nanoenvironments to the molecule that are soluble in solvents (using polar aprotic or non-polar solvent), while solidstate fluorescence can also be regarded since the restricted conformation of the molecule promotes an enhanced quantum yield. Since ESIPT is always accompanied by a diminished fluorescence quantum yield, the problem has taken the interest of Zhang et al. They addressed the issue by undertaking the role of the H-donor nitrogen in imidazole, which usually has a higher quantum yield by one order of magnitude than that of benzoxazole and benzothiazole. Besides, imidazole or benzimidazole promotes more intermolecular interactions, which is beneficial for molecular aggregations. ${ }^{83}$ 3-(4,5-Diphenyl- $1 \mathrm{H}$ imidazol-2-yl)naphthalen-2-ol (DPIN) was designed after examining two phenyl tails on imidazole that can restrict intermolecular $\pi-\pi$ stacking, thereby inhibiting aggregation-caused fluorescence quenching. When dispersing DPIN in water, stable homo-dispersed aggregated nanoparticles were formed, showing an almost exclusive keto emission with a quantum yield of 0.20 . More fascinating quantum yields were observed in DMF and DMF : water with keto emission values of 0.28 and 0.39 , respectively. The fluorescence performance of DPIN was stable in the physiological $\mathrm{pH}$ range of 6.2-9.2 without interference from common ions, making DPIN suitable as a sensing material. Additional improvement for a high fluorescent quantum yield with ESIPT character was demonstrated by Mutai et al. by spin-coating $0.5 \mathrm{wt} \%$ of the fluorophores of interest within a polymer matrix (PMMA) ${ }^{84}$ ESIPT molecules in a polymer matrix produced fluorescence with enhanced and more efficient quantum yield $\left(\Phi_{\mathrm{F}}=0.6\right)$, as compared to that in fluidic forms. Herein, we reviewed the criterion to which an ESIPT mechanism can occur within a molecule. The crucial prerequisite is the formation of a tautomer in the excited state that would red shift the emission wavelength further, leading to a large or mega Stokes shift feature.

\subsection{Intramolecular charge transfer (ICT)}

Another way to design a material with a large Stokes shift property involves the ICT mechanism, which is usually established within a molecule with a donor-acceptor (D-A) electronic system. This single emitter system is capable of revealing unique two or multi-color emissions, caused by the change in the twisting angles between the D and A units. ${ }^{85}$ Generally, organic molecules with extended $\pi$-conjugation, end-capped by $\mathrm{D}$ and A offer a facile synthetic route, molecular arrangement and unique luminescence properties that become notable in the field of material science. ${ }^{86}$ The promising avenue of the ICT mechanism in such system has led to the development of thermally activated delayed fluorescence (TADF) materials, which could maneuver future emitters in the field of OLED. D$\pi-\mathrm{A}$ molecules represent a sub-class known as the push-pull dye due to the dispersed polarization during ICT. There are many push-pull dyes present in the literature, but only a few classes will be discussed in this review. The key approach to evaluating the ICT mechanism is to skim the D and A moieties in the molecular structure.

The most developed arrangements are linear $(\mathrm{D}-\pi-\mathrm{A})$, quadrupolar $(\mathrm{D}-\pi-\mathrm{A}-\pi-\mathrm{D} \text { or } \mathrm{A}-\pi-\mathrm{D}-\pi-\mathrm{A})^{28}$ and octupolar/ tripodal $\left((\mathrm{D}-\pi)_{3}-\mathrm{A}\right.$ or $\left.(\mathrm{A}-\pi)_{3}-\mathrm{D}\right)$ systems. A typical $\mathrm{D}$ is represented by substituents like $\mathrm{OH}, \mathrm{NH}_{2}, \mathrm{OR}, \mathrm{NR}_{2}$, or heterocyclic moieties like thiophene and carbazole. On the other hand, $\mathrm{A}$ usually features substituents like $\mathrm{NO}_{2}, \mathrm{CN}, \mathrm{CHO}, \mathrm{CF}_{3}, \mathrm{SO}_{3} \mathrm{R}$, and electron-deficient heterocycles like imidazole, quinoline and benzothiazole. ${ }^{86}$ Halogens like F, Cl, Br and I can be classified as weak A. In this regard, selected systems of phenothiazine, carbazole, pyridine and rhodamine will be discussed in this section. Tanaka and his co-workers introduced a very efficient up-conversion mechanism of excitons from the spin-triplet $\left(\mathrm{T}_{1}\right)$
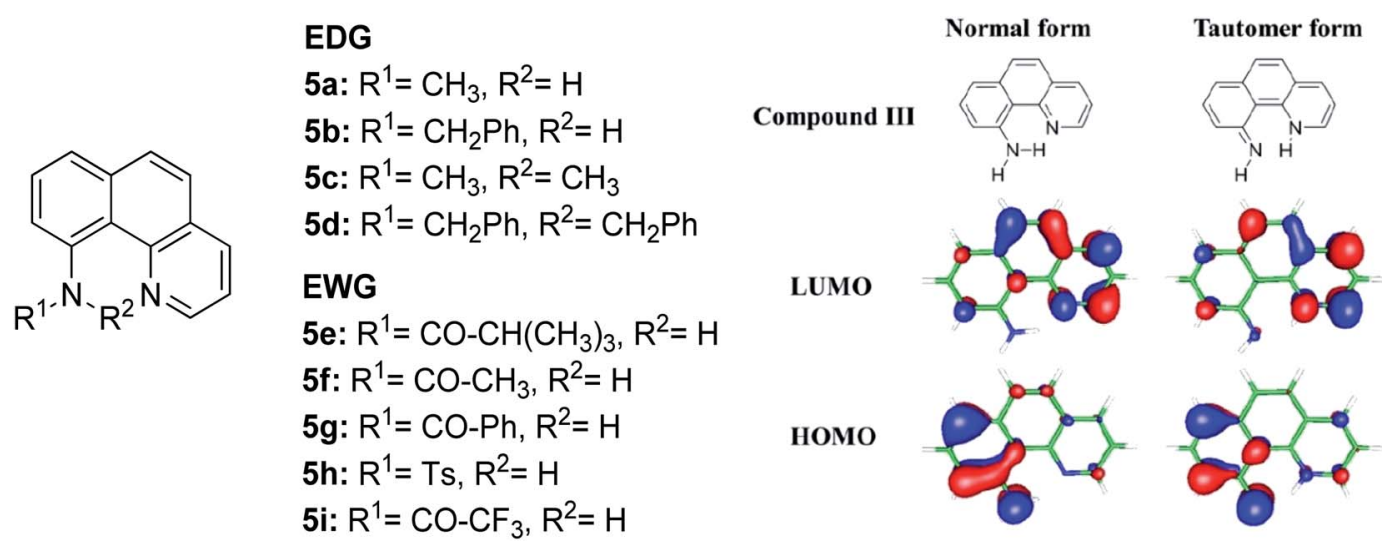

Fig. 12 Molecular structures of 10-aminobenzo[h]quinoline derivatives (left), and calculated HOMO and LUMO molecular orbitals of the normal and tautomer forms of unsubstituted compound 5 (right). The figure was adapted from ref. 81 with copyright permission from The Royal Society of Chemistry. 
<smiles>[R]N1COC(=O)c2c(C(N)=O)cccc21</smiles>

\section{Tautomer}

6a: $\mathrm{R}=\mathrm{SO}_{2} \mathrm{CH}_{3}$

6b: $\mathrm{R}=\mathrm{SO}_{2} \mathrm{CF}_{3}$

6c: $\mathrm{R}=\mathrm{COCH}_{3}$

6d: $\mathrm{R}=\mathrm{COCF}_{3}$

Fig. 13 Chemical structures of phthalimide of the normal amide form and its tautomer. The compounds are from ref. 82.

to spin-singlet $\left(\mathrm{S}_{1}\right)$ charge transfer excited state ${ }^{87}$ through the phenothiazine-triphenyltriazine (PTZ-TRZ) derivative as shown in Fig. 14. The HOMO and LUMO in such molecular system are said to be effectively separated to allow the transfer of the triplet excitons through a small energy gap. The PTZ-TRZ molecule showed a broad absorption and emission, arising from the twisted ICT state. Two emission signals with peaks around 409 and $562 \mathrm{~nm}$ were generated when excited at $340 \mathrm{~nm}$. The large Stokes shift at $562 \mathrm{~nm}$ emission was attributed to the ICT emission from the spin-singlet excited state $\left(\mathrm{S}_{1}\right)$ to spin-singlet ground state $\left(\mathrm{S}_{0}\right)$. Pivoting to the phenothiazine system as well, Zhan et al. synthesized a phenothiazine-based benzothiazole (PVBT) system that was able to emit light from $483 \mathrm{~nm}$ (in $n$ hexane) to $580 \mathrm{~nm}$ (in DMF).$^{88}$ With increasing solvent polarity, the Stokes shifted too, increasing from $9599 \mathrm{~cm}^{-1}$ to $11913 \mathrm{~cm}^{-1}$. The broadening of the lower energy emission maximum indicated an ICT characteristic. PVBT further displayed superiority in its photoluminescence property with a maximum quantum yield of 0.76 in cyclohexane. A lower $\Phi_{\mathrm{F}}$ was found in DMF (0.02), and was associated with a strong ICT process and dipole-dipole interaction between the molecules. Verification of the ICT process in PVBT was done using the TDDFT method, where they found that the HOMO was mainly distributed over the electron donor (phenothiazine unit), while the LUMO was concentrated on the electron acceptor benzothiazole unit.

Among the acceptor units, quinoline derivatives are often investigated due to their high thermal and chemical stability, high optical responses, and efficient electron transport properties. A recent study by Slodek et al. exploited the role of quinoline in comparing the fluorene's and carbazole's electron donating character. $^{89}$ The construction of 2,4-difluorenylquinoline derivatives has successfully produced bright emission in the blue spectral region $(\sim 400 \mathrm{~nm})$ and high fluorescence efficiency in solution (63-97\%). The carbazolesubstituted compound showed a strong solvent effect on the emission wavelength with increasing Stokes shift value of up to $6366 \mathrm{~cm}^{-1}$ in more polar solvent. This corroborated with the phenomenon of ICT occurring between the D-A units. The study of the carbazole units was also emphasized by Yadav $e t a l$. by comparing the strength of the donor units between triphenylamine and carbazole..$^{90}$ The absorption and emission bands of the triphenylamine-coumarin derivatives were found to be slightly red-shifted compared to that of the carbazole-coumarin derivatives. Generally, large Stokes shifts were obtained from (a)

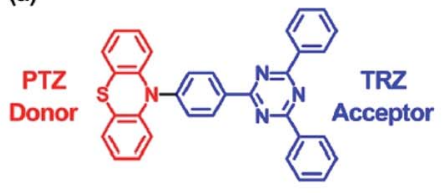

(b)
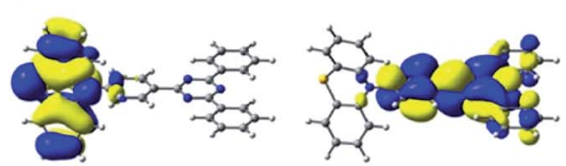

номо

"Quasi-equatorial"

(c)

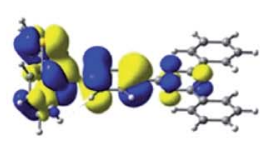

HоMо

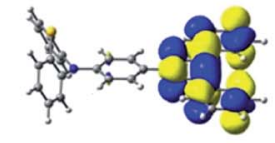

LUMO

"Quasi-axial"

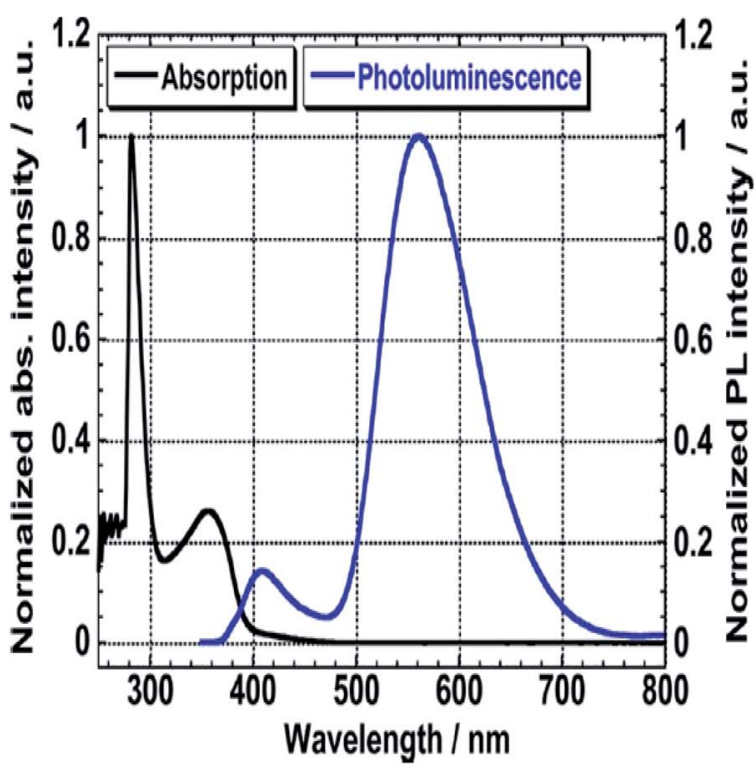

Fig. 14 (Left) (a) Molecular structures, and (b and c) HOMO and LUMO of the ground state quasi-equatorial and quasi-axial conformers of PTZTRZ calculated at the CAM-B3LYP/cc-pVDZ level, respectively. (Right) Normalized absorption (black line) and PL (blue line) spectra of PTZ-TRZ in toluene solution with a concentration of $1.0 \times 10^{-5} \mathrm{M}$. The PL spectrum was obtained by excitation at $340 \mathrm{~nm}$. The figures were reproduced from ref. 87 with permission. Copyright 2014 American Chemical Society. 

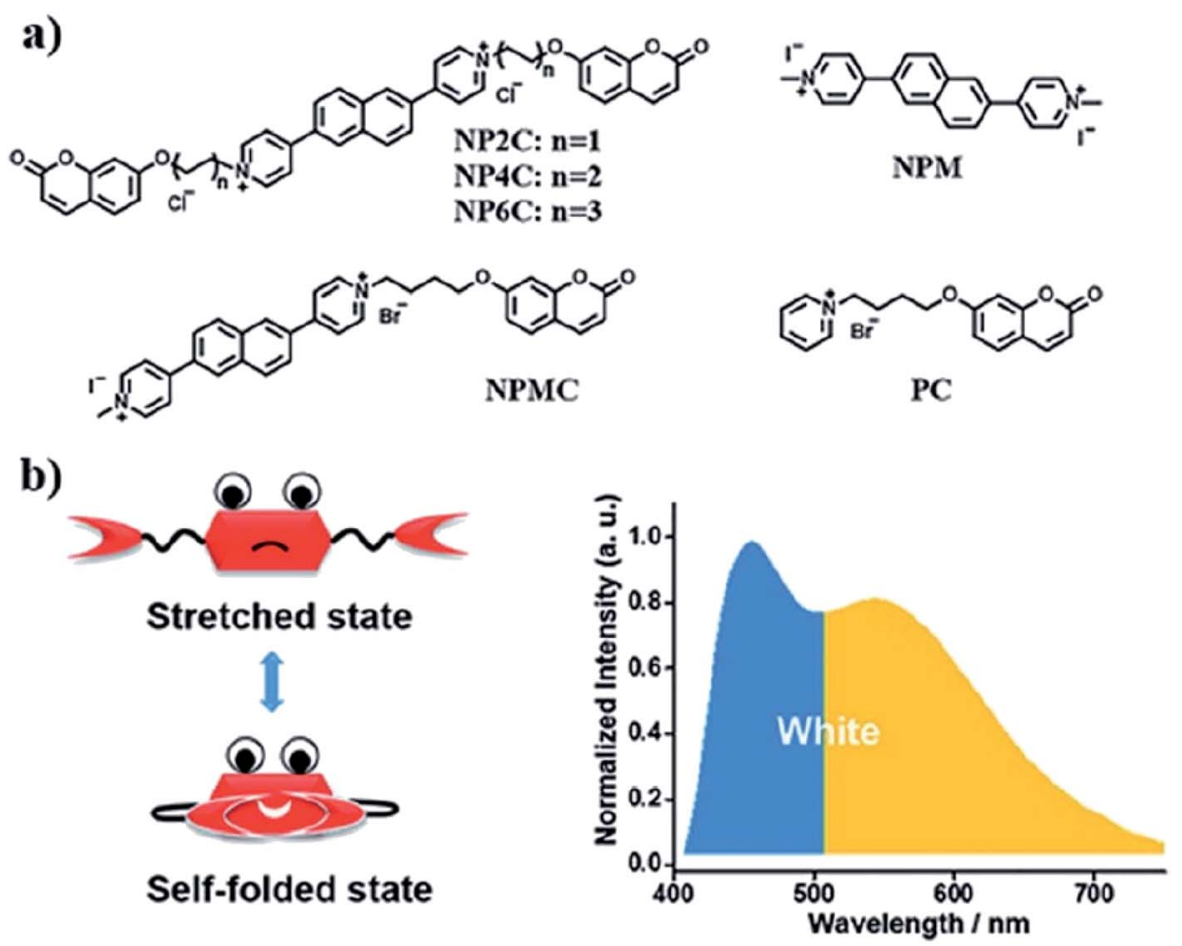

Fig. 15 (a) Molecular structures of white-light emitting organic molecules (NP4C and NP6C) and the reference compounds (NP2C, NPM, NPMC and PC); (b) the schematic cartoon illustration of single-molecule white-light emitters. The figure was reproduced from ref. 92 with copyright permission from The Royal Society of Chemistry and accreditation given to CC BY-NC 3.0.

both types of derivatives (81-135 $\mathrm{nm}$ ). The strength of the electronic ICT in D- $\pi-\mathrm{A}$ dyes can be measured to predict the solvatochromism using the generalized Mulliken-Hush (GMH) method. Positive solvatochromism is achieved when a highly polarized excited state is created. There are two ways to facilitate polarization of a molecule, i.e., to engineer the structural framework and substituents present in a molecule, and controlling its nanoenvironments (solvent, $\mathrm{pH}$, temperature, presence of dissolved oxygen, concentration).

Recently, Zhu and co-workers developed an ICT-based fluorescent probe with a large Stokes shift for determining the highly active alkali, hydrazine. ${ }^{91} 4$-(2-(3-(Dicyanomethylene)-5,5dimethylcyclohex-1-enyl)phenyl)4-bromobutanoate (DDPB) was synthesized as the sensing material that exhibited a large Stokes shift $(186 \mathrm{~nm})$. The ICT pathway facilitates the connection between the external reactive groups with the donor or acceptor moiety of the sensing material to obtain a measurement proportional to the dual emission wavelength. The substitutional cleavage cyclization reaction between the DDPB and hydrazine in their study promotes a trailblazing idea among researchers to design a single emitter that allows the ICT process to occur either intermolecularly or intramolecularly (accounting for the targeted white light emission) for a more red-shifted emission.

A new bi-functional organic molecule with a symmetric D-A-D type structure was designed by $\mathrm{Li}$ et al. using pyridinium-naphthalene (PN) core connected to two coumarin moieties via flexible alkyl chains. ${ }^{92}$ NP4C (Fig. 15), which exhibits the natural self-folded conformation, could enhance the ICT efficiency, leading to a broad emission spectrum. NP4C and NP6C were reported to be capable of emitting white light with a CIE coordinate of $(0.30,0.33)$, arising from their dualemissive properties when tested in water. This can be rationalized by the different existing chromophores at distinct regions in both compounds, which can construct a significant difference in the frontier molecular orbitals. For NP4C, the HOMO was primarily located in the coumarin groups, while the LUMO was distributed on the PN core in favor of the ICT process. The presence of the varying flexible alkyl chain lengths operates the rotation and motion of the coumarin moieties at both ends, which might lead to stacking with the PN core due to hydrophobic effects, $\pi-\pi$ stacking and donor-acceptor interactions, which further favor the CT emission. The interesting output from this research is the fact that intramolecular motions and further accelerated transformation between the self-folded and stretched states of NP4C can be achieved by gradually increasing the temperature of the aqueous solution. A similar palette was attempted by Gandioso and his group, where a compound emitting in the NIR and a large Stokes shift (99 $\mathrm{nm}$ ) were accomplished. ${ }^{93}$ A pyridinium moiety was attached at the 2-position of a coumarin core, which acted as the pull unit, inducing a more polar environment to the coumarin scaffold in the ground state. This occurrence blue-shifted the absorption band of the overall molecule, while the emission band stayed the same due to its insensitivity to solvent changes. Based on the NMR spectroscopy, the existence of $E$ and $Z$ rotamers in solution was confirmed, and they were responsible for further intensifying the push-pull effect. 
If we are to explore the reasoning of dual fluorescence that comes from small molecules, Lippert's viewpoint would be conspicuous to consider. Donor and acceptor units linked together will have a limited degree of freedom. Therefore, the most preferred vibrational mode for a relaxation involving a change in the electronic structure of the excited state is the large amplitude torsional motion or twist. ${ }^{94}$ The features of the phenomenon are further described to be strongly dependent on the surrounding conditions, like the polarity of the solvent and the thermodynamic effect. The twisted intramolecular charge transfer (TICT) has been discussed by Zhang et al. by demonstrating the photophysical process of aniline-substituted rhodamine analogues using DFT/TDDFT approaches. ${ }^{95}$ Fig. 16 presents the possible stable isomers of compound 7, 9-(2'-carboxylphenyl)-6-( $N, N$-diethylamino)-4-( $n$-phenyl-methanamine)1,2,3-trihydroxanthylium. The isomers $7 \mathbf{c}-\mathbf{f}$ are said to be twisted, unlike $\mathbf{7 a}$ and $\mathbf{7 b}$, which are completely planar. The geometry of the relaxed $\mathrm{S}_{2}$ excited state of $7 \mathrm{c}$ shows that the anilino moiety is planar to the xanthene plane, suggesting that TICT may occur in this isomer. Compound 7 possesses rather red-shifted absorption bands and simultaneously, a red-shifted emission wavelength $(\sim 600 \mathrm{~nm})$. TICT is responsible for the
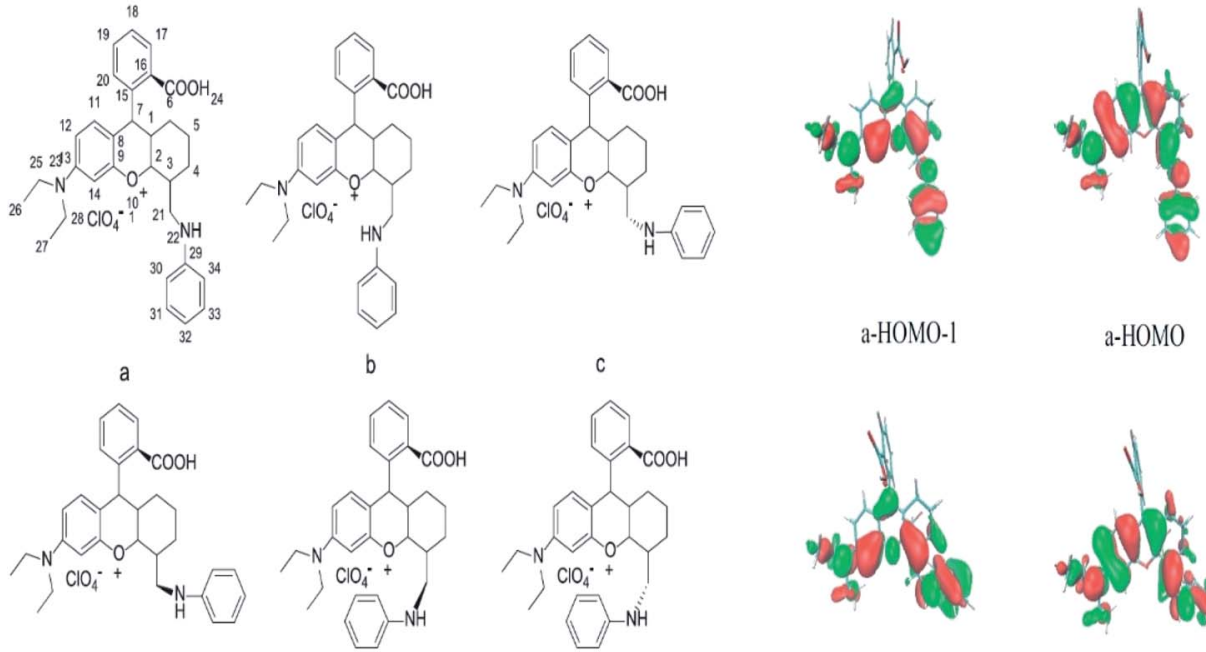

a-HOMO-1
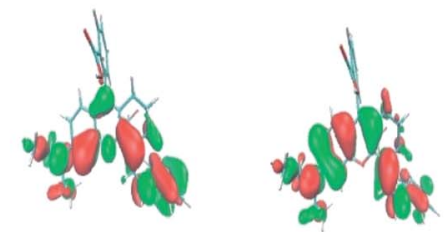

c-HOMO-1

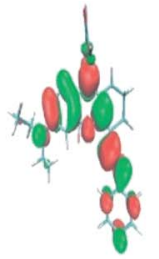

a-LUM0

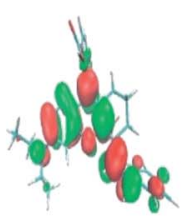

c-LUM0
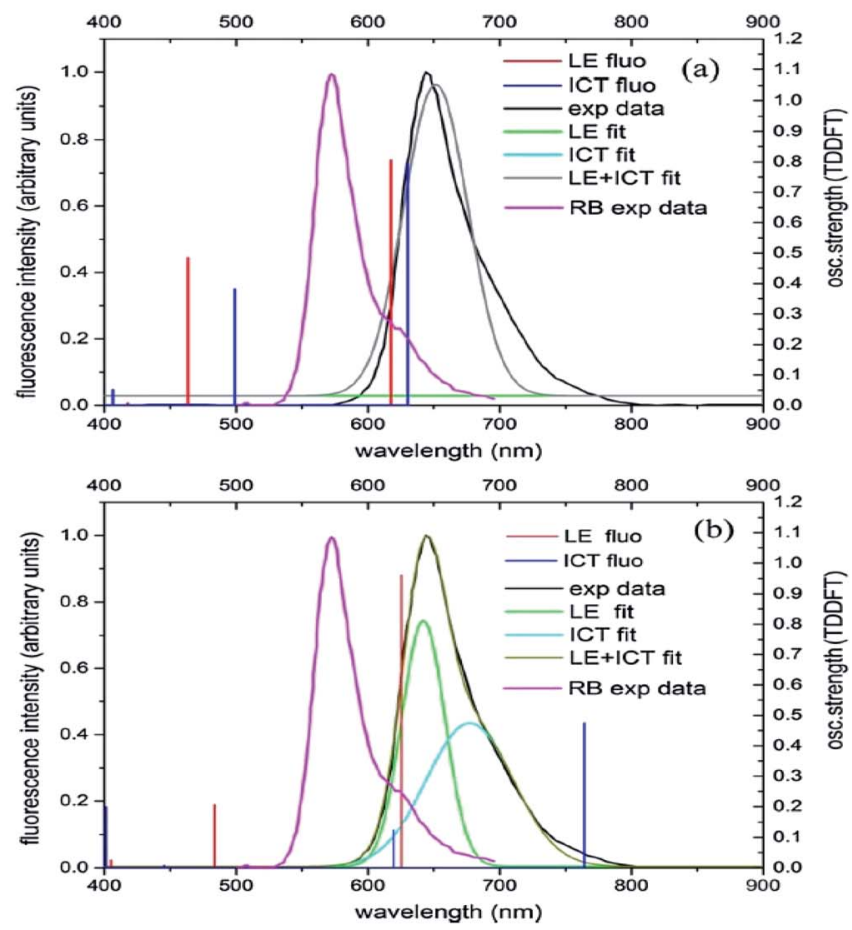

Fig. 16 Six stable isomers for anilino-substituted Rhodamine analogues and visualization of HOMO-1, HOMO and LUMO+1 of the complex 7 (top). Experimental and theoretical fluorescence spectra, the deconvoluted local excited state (LE) and ICT bands for (a) 7a; (b) 7c (bottom). The figures were reproduced from ref. 95 with copyright permission from Elsevier. 
Table 1 Photophysical properties of some ICT-active molecules<smiles>CN(C)c1ccc(-c2cc(-c3ccccn3)nc(-c3ccccn3)c2)c2nonc12</smiles>

8<smiles>c1ccc(N(c2ccccc2)c2ccccc2)cc1</smiles><smiles>c1ccc(-c2cccc(-c3ccccn3)n2)nc1</smiles><smiles>CCC1=C(C)C2=C(c3ccccc3)c3cc4c(=O)oc5cc(N(CC)CC)ccc5c4n3S(F)(F)B(F)n3c(C)c(CC)c(C)c3C2=C1</smiles>

279, 336, $470\left(\mathrm{MeCN}: \mathrm{H}_{2} \mathrm{O}\right)$

624

0.036

-

33

354 (n-hexane)

$360\left(\mathrm{CH}_{2} \mathrm{Cl}_{2}\right)$

$357(\mathrm{MeCN})$

$362(\mathrm{EtOH})$

$359(\mathrm{MeOH})$

$620(\mathrm{CyH})$

632 (PhMe)

$602\left(\mathrm{CH}_{2} \mathrm{Cl}_{2}\right)$

$590(\mathrm{MeOH})$

606 (DMF)

\section{$620(\mathrm{CyH})$ \\ $606\left(\mathrm{CH}_{2} \mathrm{Cl}_{2}\right)$ \\ $594(\mathrm{MeOH})$ \\ 608 (DMF)}

$343\left(\mathrm{CH}_{2} \mathrm{Cl}_{2}\right)$
393 (n-hexane)

$476\left(\mathrm{CH}_{2} \mathrm{Cl}_{2}\right)$

489 (MeCN)

505 (EtOH)

$524(\mathrm{MeOH})$
$643(\mathrm{CyH})$

632 (PhMe)

$697\left(\mathrm{CH}_{2} \mathrm{Cl}_{2}\right)$

$720(\mathrm{MeOH})$

748 (DMF)
$0.86(\mathrm{CyH})$

0.62 (PhMe)

$0.41\left(\mathrm{CH}_{2} \mathrm{Cl}_{2}\right)$

$0.06(\mathrm{MeOH})$
0.04 (DMF)
40

$\begin{array}{lll}642(\mathrm{CyH}) & -(\mathrm{CyH}) \\ 706\left(\mathrm{CH}_{2} \mathrm{Cl}_{2}\right) & 0.31\left(\mathrm{CH}_{2} \mathrm{Cl}_{2}\right) & \\ 724(\mathrm{MeOH}) & -(\mathrm{MeOH}) & - \\ 752(\mathrm{DMF}) & 0.03(\mathrm{DMF}) & -\end{array}$

40

$431\left(\mathrm{CH}_{2} \mathrm{Cl}_{2}\right) \quad 0.63\left(\mathrm{CH}_{2} \mathrm{Cl}_{2}\right) \quad 2.57\left(\mathrm{CH}_{2} \mathrm{Cl}_{2}\right) \quad 31$

References 
Table 1 (Contd.)

\begin{tabular}{llllll}
\hline Compound & $\lambda_{\text {abs }}(\mathrm{nm})$ & $\lambda_{\text {em }}(\mathrm{nm})$ & $\Phi_{\mathrm{F}}$ & $\tau(\mathrm{ns})$ & References \\
\hline
\end{tabular}

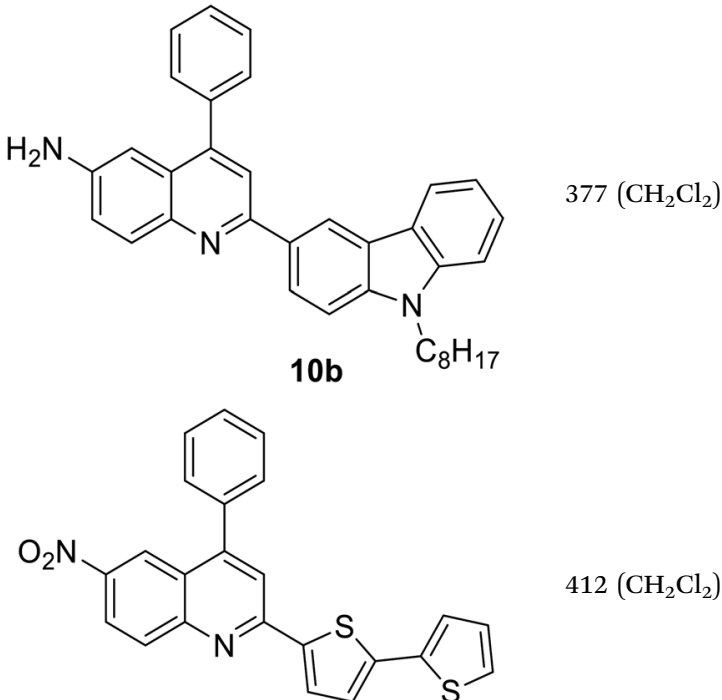

$11 \mathrm{a}$<smiles>c1csc(-c2ccc(-c3cc(-c4ccc(-c5cccs5)s4)c4ccccc4n3)s2)c1</smiles>

11b
$380\left(\mathrm{CH}_{2} \mathrm{Cl}_{2}\right)$

$479\left(\mathrm{CH}_{2} \mathrm{Cl}_{2}\right)$

$0.53\left(\mathrm{CH}_{2} \mathrm{Cl}_{2}\right)$

$1.13\left(\mathrm{CH}_{2} \mathrm{Cl}_{2}\right)$

31 and 97 large Stokes shift of compound 7 due to the presence of a strong electron-withdrawing anilino unit within the rhodamine precursor.

Based on the discussed examples, we have shown that the addition of specific substituted groups and modulating steric restrictions from eith-er bulkiness or $\pi-\pi$ interactions can shift the absorption and emission simultaneously. Tuning the ICT process in a push-pull system becomes eminent in regulating the charge distribution over a molecule. Effects of the $\mathrm{NO}_{2}$ group have been covered by Panja and his research group, where they varied the number and position of $\mathrm{NO}_{2}$ groups that exist in a phenolic core. It was discovered that one strong and highly feasible ICT process happened in the para position of nitrophenolate that produced absorption at $420 \mathrm{~nm}$ (in acetonitrile). The ICT character increased when the number of $\mathrm{NO}_{2}$ groups increased in the molecule. This minute alteration changed the transition orbitals from HOMO to LUMO and HOMO to LUMO+1.96 Transfer of electronic charge fractions between the molecular entities causes a huge loss of excitation energy, resulting in large Stokes shift phenomenon. Certainly,
ICT is one of the encouraging approaches to consider when developing a white light emitter with large Stokes shift properties. In order to generate a true white light, detailed photophysical mechanisms of the molecule need to be understood, simulated and designed prior to synthesis execution. The details of photophysical properties such as maximum absorption and emission wavelength, fluorescence quantum yield and fluorescence lifetime of some ICT-active molecules introduced in this review are summarized in Table 1.

\subsection{Excited state geometry relaxation and structure deformation}

The designation of molecules with a large Stokes shift property involves compromise on the drawback of self-absorption. Selfabsorption is due to the proportions of the fluorescence wavelength being re-absorbed by the same or other molecules for subsequent fluorescence at a longer wavelength region. To address this issue, the de novo approach is to increase the geometry relaxation of the molecular framework upon photoexcitation. This method has been described by Chen et al. in 
their study by introducing thienyl substituents at the 2- and 6position of a BODIPY core (12a and 12b).${ }^{98}$ The output from DFT calculations proved that there was a change in the dihedral angle of the compound (between the thienyl unit and the BODIPY core) from $59^{\circ}$ to $39^{\circ}$ when comparing the geometry in the $S_{0}$ and $S_{1}$ states. The delineation for this is an increased difference between the emission energy as compared to the excitation energy that subsequently led to an enlarged Stokes shift. The excitation and emission maxima for 12a were at $509 \mathrm{~nm}$ and $601 \mathrm{~nm}$, respectively, giving a Stokes shift value of $92 \mathrm{~nm}$, while 12b had a Stokes shift value of up to $106 \mathrm{~nm}$, giving a deep red fluorescence (Fig. 17). Other than assessing the dihedral angle changes in the excited state, recognizing any sterically hindered or restrained part of a molecule could shine light in the large Stokes shift material designation. Doroshenko et al. studied the ortho analogues of alternating the phenyloxazole-phenyl-oxazole-phenyl (POPOP) ring, which induced high steric hindrance and nonplanarity of the molecule. ${ }^{99}$ Such conformation and arrangement flattened the excited singlet state of the molecule, which further lowered the fluorescent energy and in turn, gave an enlargement of the Stokes shift. POPOP generally has an intramolecular interaction nature due to the bulkiness of the moieties, and thus experiences a minor influence of the environments. When the oxazole ring was replaced by oxadiazole instead, a nearly doubled flattening of the activation energy was observed, meaning a larger Stokes shift was achieved. This happened because the light was mainly absorbed by the phenyl-oxadiazolyl fragment, causing the molecule to rotate out of the main plane due to the sterically hindered oxadiazolyl in the electronic absorption spectra. The fluorescence quantum yield observed for POPOP derivatives were reasonably high, from 0.5 to 0.6 , depending on the solvent used.

Synthetic interest in cyanine dye can be associated with its high molar extinction coefficients, good fluorescence quantum yield and easy molecular tuning. In this regard, Sissa et al. studied the extension of the polymethine chain and introduction of the cyclohexenyl ring in the central position of the cyanine dye. ${ }^{\mathbf{1 0 0}}$ It was understood that such molecular modification was responsible for the huge Stokes shift (larger than 0.3 eV) observed in their study. Through investigation of the first excited state and of its geometrical relaxation upon photoexcitation, a cyanine derivative encompassing an amino substitution on the heptamethine spacer was steered to a brokensymmetry ground state, which was subsequently restored upon relaxation in the excited state. This phenomenon caused a large structural re-arrangement of the molecule, and thus justifies the large Stoke shift seen in dichloromethane and DMF solvents. Extension of the $\pi$-conjugation framework of coumarin by integrating the benzothiazole moiety has also become one of the renowned strategies to aim for long emission wavelength. Huang and his co-workers devised new coumarin structures that are capable of undergoing structural deformation in their excited state. ${ }^{\mathbf{1 0 1}}$ Based on the TDDFT method with optimized excited state geometries $\left(\mathrm{S}_{1}\right.$ state), geometrical changes were realized for three of the derivatives (13a, 13b and 13c) in Fig. 18. As for 13a, the dihedral angle between the benzothiazole moiety and fused coumarin moiety changed from $30^{\circ}$ in the $S_{0}$ state to a coplanar conformation of $0^{\circ}$ in the optimized $S_{1}$ state. Based on this significant geometry relaxation, the energy levels of the LUMO orbital decreased significantly too, giving rise to a large Stokes shift $(87 \mathrm{~nm})$ of the compound. Since there was not much difference in the variation of the dihedral angles in $\mathbf{1 3 \mathbf { b }}$, this compound has a lower Stokes shift value of $81 \mathrm{~nm}$. Surprisingly, 13c showed the largest Stokes shift of $144 \mathrm{~nm}$ with yellow emission $\left(\lambda_{\mathrm{em}}=555 \mathrm{~nm}\right)$ due to the presence of an acetylide phenyl moiety. In both $S_{0}$ and $S_{1}$ states, both HOMO orbitals were localized on this acetylide phenyl moiety, while the LUMO orbitals were more localized on the fused coumarin moiety. Aside from having geometry changes within the molecule, ICT played an important role in producing the large Stokes shift for this compound.

The interest in the naphthalene-fused BODIPY dye synthesis by Yang et $a{ }^{102}$ has led to the discovery of its asymmetric analogue that exhibits a large Stokes shift and high photostability. By investigating the frontier molecular orbital profiles after geometry optimization using TDDFT, it has been proven again that the geometrical relaxation of the molecule is one of the promising avenues that researchers should consider when designing large or mega Stokes shift compounds. Upon photoexcitation, there was a decrease in the LUMO energy level of the derivative from $-2.56 \mathrm{eV}$ (in the Franck-Condon state) to $-2.93 \mathrm{eV}$ (in the optimized $\mathrm{S}_{1}$ state), which accounts for the large Stoke shift characteristic. Kushida and co-workers also worked on a constraint-induced structural deformation in the excited state, particularly for the initially planarized triphenylborane derivatives. ${ }^{103}$ An important concept in molecular engineering is to provide a structural constraint to the $\pi$ skeleton. This will restrict the structural flexibility, thereby suppressing nonradiative decay pathways from the electronically excited state. Since boron has a vacant $\mathrm{p}$ orbital, $\pi$-conjugation can be prompted by linking boron with electron acceptor materials. In Kushida's study, threemethylene-bridged triphenylboranes showed unique excited state dynamics, including plane-to-bowl structural changes, dual emission at $337 \mathrm{~nm}$ and $407 \mathrm{~nm}$, and reasonably good fluorescence yield. The bowl-shaped structure in the $S_{1}$ state was accountable for the red-shifted and lower energy emission.

In the case of the inorganic fluorescent dye, the role of structure deformation is responsible for the broadband emission. Applying the same concept as geometry relaxation, hybrid perovskites $(\mathrm{NBT})_{2} \mathrm{PbI}_{4}$ and $(\mathrm{EDBE}) \mathrm{PbI}_{4}$ were investigated to undergo structural changes in the HOMO-LUMO transition of the $\mathrm{I}_{3}{ }^{-{ }^{-104}}$ The emergence of such states was associated with emissive traps, leading to large Stokes-shifted luminescence in accordance with the relaxation mechanism typical of trapped charge carriers. These behaviors were also reported in self-assembled organicinorganic perovskite $\left(\mathrm{C}_{6} \mathrm{H}_{11} \mathrm{NH}_{3}\right)_{2} \mathrm{PbBr}_{4}$ that underwent elastic deformation for exciton delocalization to produce strongly Stokesshifted emission ${ }^{\mathbf{1 0 5}}$ and perovskite ( $N$-MEDA) $\left[\mathrm{PbBr}_{4}\right]$ that weathered elastic deformation, leading to strong vibronic coupling between excitons and the lattice to induce a Stokes shift of up to $170 \mathrm{~nm} .{ }^{16}$ We have concluded the excited state structural or geometrical dynamics that can lead to a distinct gap between the absorption and emission wavelengths based on the reviewed 
<smiles></smiles>

$12 \mathrm{a}$<smiles></smiles>

$12 b$
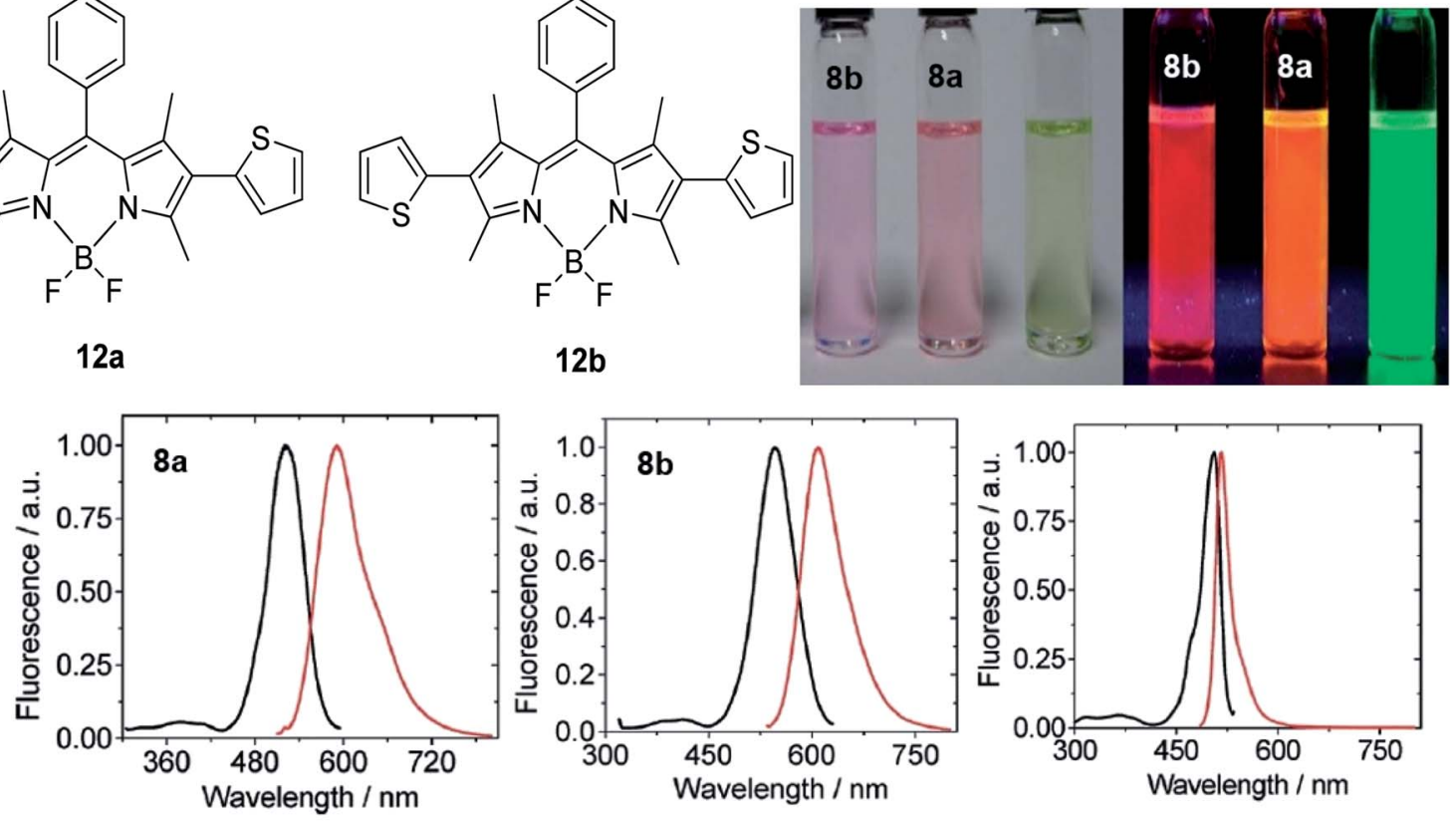

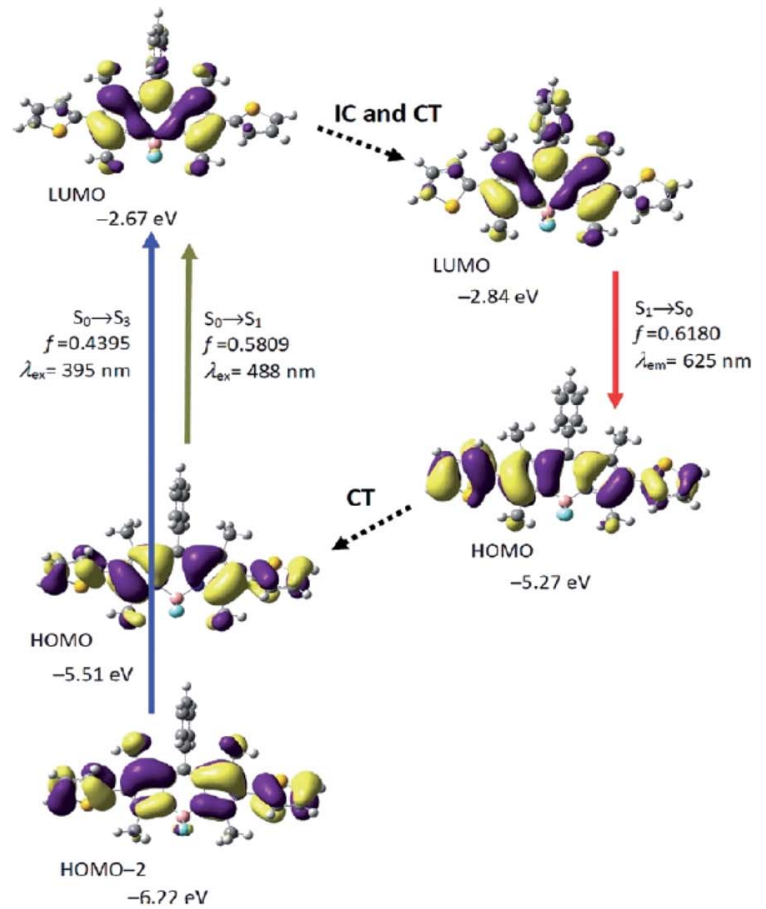

Fig. 17 (Top) Molecular structure of 2-thienyl (12a) and 2,6-bisthienyl (12b) BODIPY derivatives and their emission color, taken under ambient light and UV light (handheld UV lamp, $365 \mathrm{~nm}$ ). $\mathrm{C}=7.5 \times 10^{-6} \mathrm{M}$ (toluene, $25^{\circ} \mathrm{C}$ ). (Middle) Fluorescence spectra of $12 \mathrm{a}, 12 \mathrm{~b}$ and unsubstituted BODIPY. (Bottom) Rationalization of the large Stokes shift of $12 \mathrm{~b}$ : the geometry relaxation upon photoexcitation, and the frontier molecular orbitals (MOs) involved in the vertical excitation (i.e., UV-vis absorption, the left two columns) and emission (right column) of 12b. The figures were adapted from ref. 98 with permission. Copyright 2012 American Chemical Society.

examples involving BODIPY, oxazole, cyanine, coumarin, triphenylborane and some inorganic perovskites.

\subsection{Hydrogen bonding mediated $\mathrm{J}$ - or $\mathrm{H}$-aggregation and aggregation induced emission (AIE)}

Another factor that governs the molecule in possessing a large Stokes shift component is the role of the aggregation morphology of the luminescent molecules. Before delving into AIE, the difference between the two types of aggregates should be noted. When dye molecules aggregate and form a concentrated aqueous solution with bound molecular assemblies, their absorption band will shift significantly towards the longer wavelength of the visible spectrum with respect to the monomer absorption band. Such phenomenon is known to be J-type 
<smiles>Cc1cc(=O)oc2c1ccc1oc(=O)c(-c3nc4ccccc4s3)cc12</smiles><smiles>Cc1cc(=O)oc2c1ccc1oc(=O)c(-c3ccc(Br)cc3)cc12</smiles><smiles>Cc1cc(=O)oc2c1ccc1oc(=O)c(-c3ccc(C#Cc4ccc(N(C)C)cc4)cc3)cc12</smiles>

$13 a$
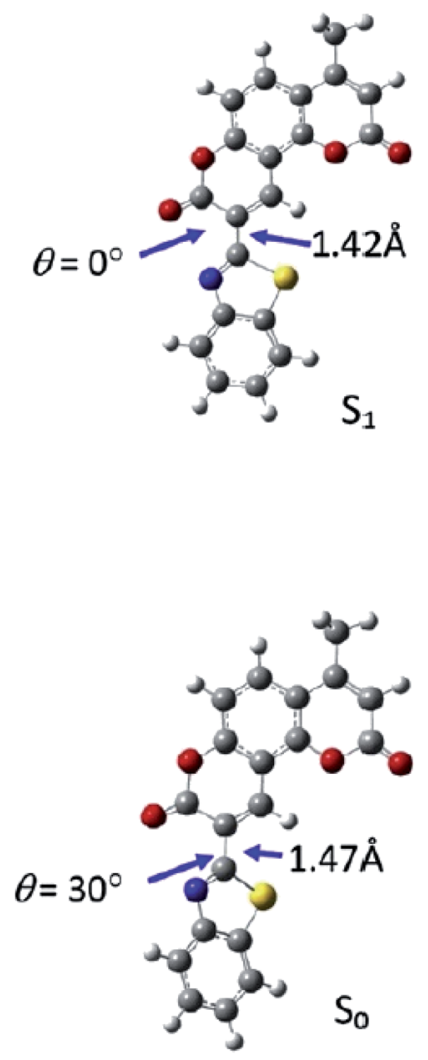

13a

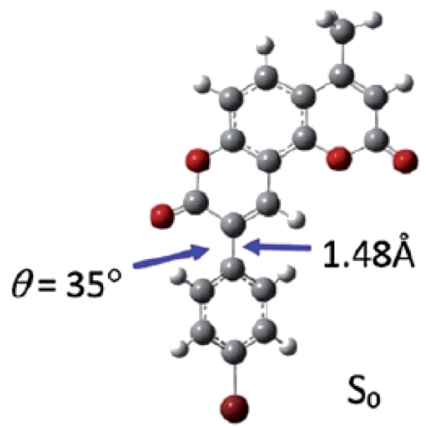

$13 b$
$13 b$

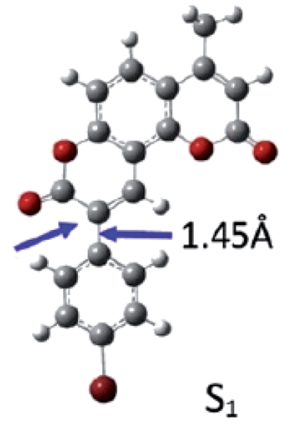

$\theta=22^{\circ}$

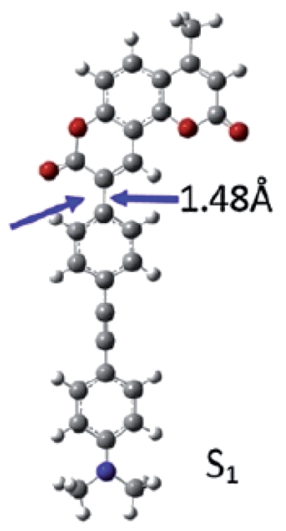

$13 c$
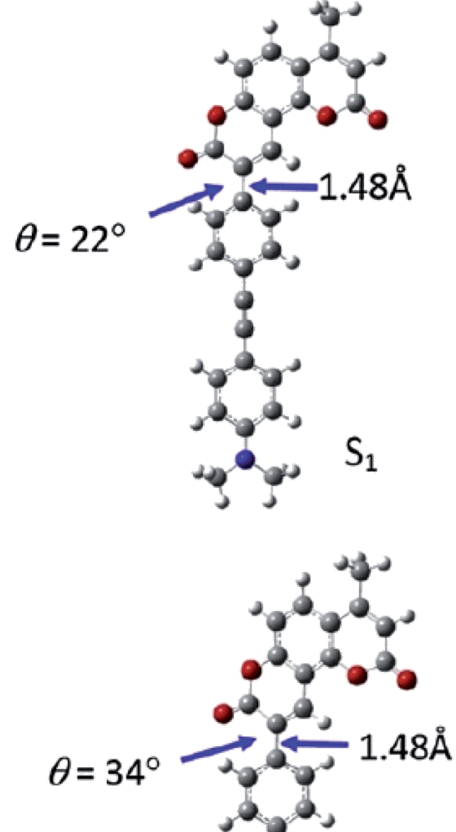

2.

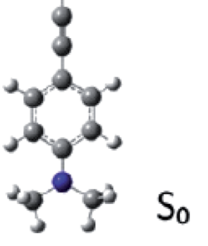

$13 c$

Fig. 18 Geometry of the coumarins $13 \mathrm{a}, 13 \mathrm{~b}$ and $13 \mathrm{c}$ at the ground state $\left(\mathrm{S}_{0}\right)$ and the singlet excited state $\left(\mathrm{S}_{1}\right)$. Toluene was used as a solvent in the calculation. Calculated at the B3LYP/6-31g(d) level with Gaussian 09W. The figure was adapted from ref. 101 with copyright permission from Elsevier. 
aggregation in honor of Jelley's (J-) and Scheibe's bathochromically shifted aggregation of pseudoisocyanine chloride discovery in the early 1930s. ${ }^{106}$ Conversely, any blue-shifted absorption bands due to the self-assemblies with different arrays (depending on the dipole moments) are termed as H-aggregate ( $\mathrm{H}$ denotes hypsochromic). ${ }^{107} \mathrm{H}$-aggregate could be one of the contributing factors to providing a large Stokes shift component, but comes with its downside of being non-emissive in most cases unless with several exceptions. Understanding the behaviors of these aggregates provides general guidance for researchers to design and control the aggregation morphology of the luminescent molecules in correspondence to their functionalities.

Concerning the nature of $\mathrm{H}$-aggregates that result from the parallel stacking of molecules with a "side-by-side" orientation, we will look into a report by Ryu et al. on the remarkably large Stokes shift that was sourced from $\mathrm{H}$-aggregated cyanine dyes. ${ }^{108}$ The system that they proposed was based on the interaction of the dye molecules with chiral assemblies of a cationic gemini surfactant. The key to obtaining a large Stokes shift molecule and narrow absorption band is to establish a media with the presence of the gemini surfactants (16-2-16 L-tartrate was used in their experiment). With this, the dyes can be electrostatically bound to the surfactants in the form of head-tohead arrangement. In methanol containing $0.2 \mathrm{mM}$ of 16-2-16 L-tartrate, the H-aggregated cyanine dyes is capable of producing a notably large Stokes shift of up to $224 \mathrm{~nm}$. This was in line with a previous study on the red-shifted fluorescence that was set forth to originate from the lower energy exciton state (forbidden state) of the H-aggregate species. ${ }^{109}$ However, Ryu and his group assumed that the $\mathrm{H}$-aggregated cyanine dyes on the 16-2-16 L-tartrate assemblies in their study were converted to an excimer-like state upon photoexcitation. The wicked aggregation-caused quenching (ACQ) effect will also transpire from the presence of a detrimental species, like the excimer and exciplex. ${ }^{110}$ Hence, the judicious utilization of the AIE effect needs to be taken into consideration, while enriching the AIE palette for different applications.

As for J-aggregates, they are known to have a small Stokes shift and red-shifted absorption band. However, such claims might vary depending on the structure-property relationships for different types of compounds. For instance, hydrogen bonding mediated J-aggregation has been mentioned by Molla et al. in their study on a single component chromophore based on the naphthalenediimide skeletal system. ${ }^{111}$ Compounds 14a and 14b were synthesized to investigate the role of the carboxyl groups that can form a self-complementary hydrogen bonding network, with the long alkyl chain for enhanced solubility in non-polar media. The absorption profile of $\mathbf{1 4 a}$ from polar to a less polar solvent unveiled a concomitant redshift band, but of reduced intensity, which was said to emanate from the J-type $\pi$ stacking. The control molecule 14b showed no significant changes in its absorption spectra, which ascertained the role of the hydrogen bonds in promoting a red-shifted spectral feature. In a mixture of solvents consisting of $95: 5$ of methylcyclohexane : chloroform, 14a was shown to have various possible hydrogen bonding motifs of the carboxyl functionalities. However, the syn-syn catemer (Fig. 19) formation was said to be the most probable mode of self-assembly that corroborates the J-aggregation-induced redshift in the absorption and emission spectra. Compound 14a was reported to emit at $545 \mathrm{~nm}$, giving a Stokes shift value of $151 \mathrm{~nm}$, indicating emission from an excimer-like species.

The behaviors of both $\mathrm{J}$ - and $\mathrm{H}$-aggregates were proven not necessarily rigid, but may have alterations based on previous studies. It is undeniable that there is still a lot of effort needed to decipher light-emitting processes at the molecular and photophysical levels. Moving onto a more general aggregation, recently in 2018, Gong and his group designed red-emitting salicylaldehyde Schiff base (SSB) dyes with AIE behaviors and large Stokes shifts. ${ }^{112}$ When the SSB dye (compound 15) was tested in a tetrahydrofuran : water mixture, it displayed a strong yellow to red fluorescence (up to $617 \mathrm{~nm}$ ) and large Stokes shift (up to $152 \mathrm{~nm}$ ). A higher water volume fraction within the solvent mixture led to fluorescence enhancement and high absolute fluorescence quantum yield (up to 70-fold when the water fraction reached $90 \%$ ). To scrutinize the aggregation state of compound 15, dynamic light scattering (DLS) was used to determine the particle size. Compound $\mathbf{1 5}$ having the smallest particle size (171.59 $\mathrm{nm}$ on average) was interrelated to the restriction of intramolecular rotation, hence possessing appreciatively high AIE activity (170.0). This coherently describes the recovered formation of intramolecular hydrogen bonds between the dye molecules (Fig. 20) that led to the notably large Stokes shift. Profound information was retrieved from the analysis of the single crystal structure of compound 15, which explains the AIE behavior in more detail. The distance of $\mathrm{O}-\mathrm{H} \cdots \mathrm{N}$ was $1.84 \AA$, proving the presence of stronger intramolecular hydrogen bonding within the molecule that also facilitated the ESIPT process. Schiff base derivatives are known to offer a myriad of applications in provision of its large Stokes shift and other propitious photophysical properties. ${ }^{113}$ Both AIE and ESIPT photophysical mechanisms are responsible for the large Stokes shift of the molecule.

In the same year, Song et al. proposed a novel AIE plus ESIPT properties fluorescent probe based on salicylaldehyde azine (SA) to overcome the small Stokes shift and aggregation-caused quenching (ACQ) problems that arise when accumulated in cell $^{114}$ and biological media. However, the focus of the discussion is the mother molecule that is capable of producing a large Stokes shift of up to $148 \mathrm{~nm}$. Like Gong's study, they reported that the AIE character of the fluorescent dye can be evaluated when investigating the parameter of the fluorescence wavelength and intensity with the increase of water volume fraction. In the solvent of the PBS buffer-DMSO system, the SA's fluorescence intensity increased sharply when the water volume fraction exceeded $80 \%$. Aggregates formed in the PBS buffer ("poor solvent" as termed by Tang et al., indicating the poor solvating power with increased water volume fraction ${ }^{115}$ ) also produced a red-shifted fluorescence, establishing a significant difference in the excitation and emission wavelengths upon cysteine recognition. A coumarin-based AIE-active red emission has been developed by Yan et al. by exploiting the capacity of the Schiff base emerging from aldehyde and amino groups. ${ }^{116}$ The fluorescence behavior in water: DMF $\left(f_{\mathrm{w}}\right.$, vol\%) mixtures 

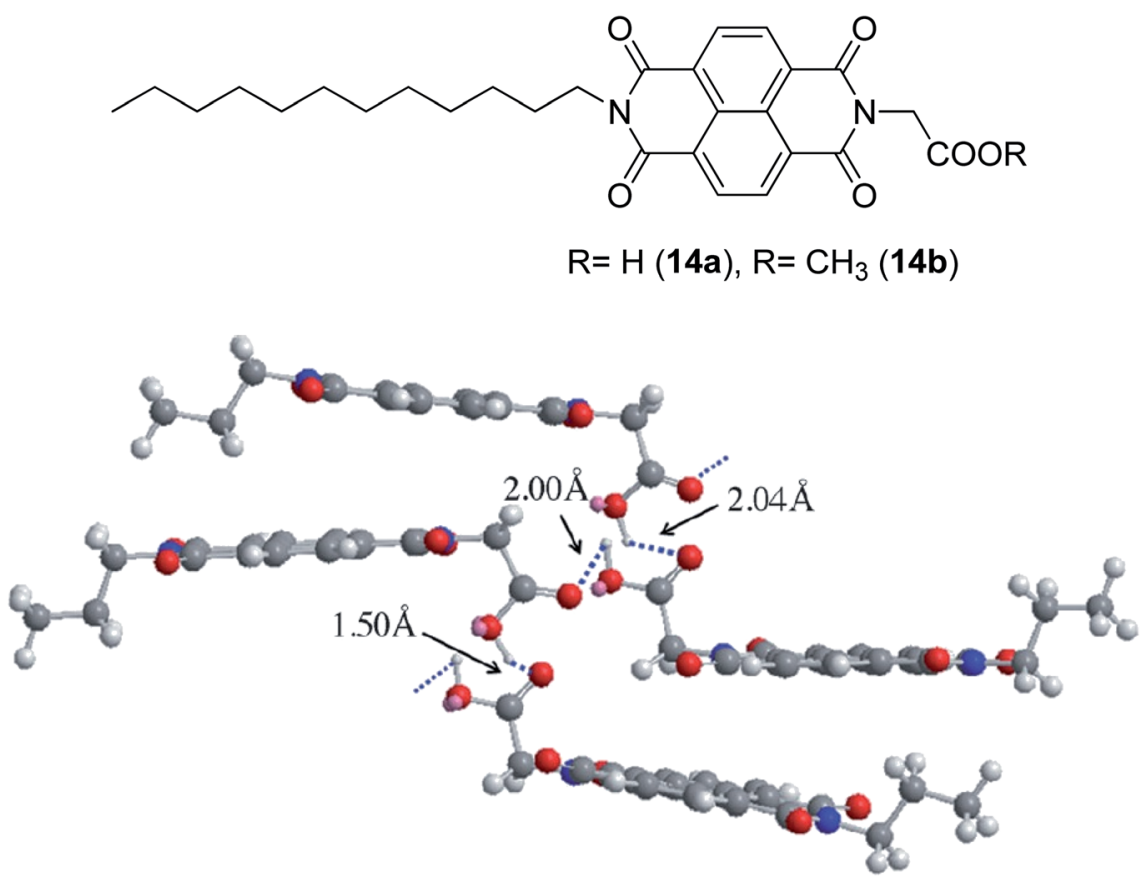

Fig. 19 1,4,5,8-Naphthalenetetracarboxdiimide derivatives (14a and $14 b)$ that were synthesized from ref. 111. The lower image shows the energy-minimised self-assembled structure of 14a (for simplicity, the alkyl chain in 14a was replaced by a $n$-propyl chain). The figure was reproduced from ref. 111 with copyright permission from John Wiley and Sons.

showed enhanced aggregation with a higher water fraction. In pure DMF $\left(f_{\mathrm{w}}=0 \%\right)$, the coumarin Schiff base showed very weak fluorescence, whereas when $f_{\mathrm{w}}$ was varied from $10 \%$ to $40 \%$, the emission intensity decreased along with a slight red shift of the emission peak as the solvent polarity increased. The behavior implied the presence of the TICT process, which caused a fluorescence decrease. The highlight of their study was when $f_{\mathrm{w}}>$ $40 \%$, and the maximum emission wavelength shifted from $540 \mathrm{~nm}$ to $640 \mathrm{~nm}$ alongside an evident increase of fluorescence intensity. This red shift was ascribed to aggregation, which induced an additional mechanism responsible for the large Stokes shift (245 nm) and enhanced emission intensity. Based on the particle size distribution study (DLS), one size distribution peak was observed at $20 \mathrm{~nm}\left(f_{\mathrm{w}}=40 \%\right)$ and two size distribution peaks were detected at $494 \mathrm{~nm}$ and $9.8 \mu \mathrm{m}$, respectively $\left(f_{\mathrm{w}}=60-70 \%\right)$. At $f_{\mathrm{w}}=90 \%$, the size of coumarin Schiff base particles shifted to larger values, thus consolidating the AIE character of the molecule. Yan's coumarin Schiff base reached a Stokes shift value of up to $146 \mathrm{~nm}, 161 \mathrm{~nm}$, and $199 \mathrm{~nm}$ in toluene, ethyl acetate and ethanol, respectively. In thin film form, the material was capable of giving red emission with $26.3 \%$ quantum efficiencies.

Therefore, the next aspect to explore is researchers' strategies to molecularly design AIE-bearing compounds and/or endow AIE molecules with self-assembly. This question has been elaborated by $\mathrm{Wu}$ et al., in which they highlighted that AIE molecules would normally be nonplanar with: (1) propellershaped molecules, (2) butterfly or V-shaped molecules, or (3) rotatable linear molecules. ${ }^{\mathbf{1 1 7}}$ On these accounts, important approaches to envisage include the presence of intermolecular interactions, like $\pi-\pi$ stacking, hydrogen bonding, van der Waals forces and hydrophobic effects. Based on the reviewed structural cores (cyanine, naphthalenediimide, salicylaldehyde and coumarin Schiff bases and salicylaldehyde azine), it is evident that the prerequisite to supplicate AIE is by accommodating a suitable solvent system and condition for molecules to start to agglomerate and fluorescence. Accompanying mechanisms like ESIPT and ICT are also liable for the preferred photoluminescence properties of AIE molecules.

\subsection{Interchange between monomer and excimer fluorescence}

Tuning fluorescent compounds to acquire a large Stokes shift characteristic is indeed challenging, especially when factors that regulate the mechanistic aspect imperil other properties that are demanded in white light generation or other lighting applications. Such feature peculiarity is reinforced in the excimer formation of a molecule. Excimers are excited atomic or molecular dimers $\left(\mathrm{A}^{*} \mathrm{-} \mathrm{A}\right)$ that are normally derived from aromatic molecules, noble gas atoms, monatomic vapors, and activator ions in alkali halide crystals. ${ }^{118}$ They are fluorescent in nature, with an emission band produced at lower energy than its monomer as a result of the proximity between multiple chromophores. ${ }^{119}$ An excimer system typically combines strong lateral bonding (covalent bonding) with weak interlayer binding. ${ }^{120}$

Highly conjugated molecular systems (e.g., naphthalene, pyrene, phenanthrene, anthracene, among others) are great excimer contributors due to the facile $\pi-\pi$ interaction. The pyrene series have been reported to produce a long emission 
<smiles>Oc1ccccc1/C=N/N=C/C=N/N=C/c1ccccc1O</smiles>

15
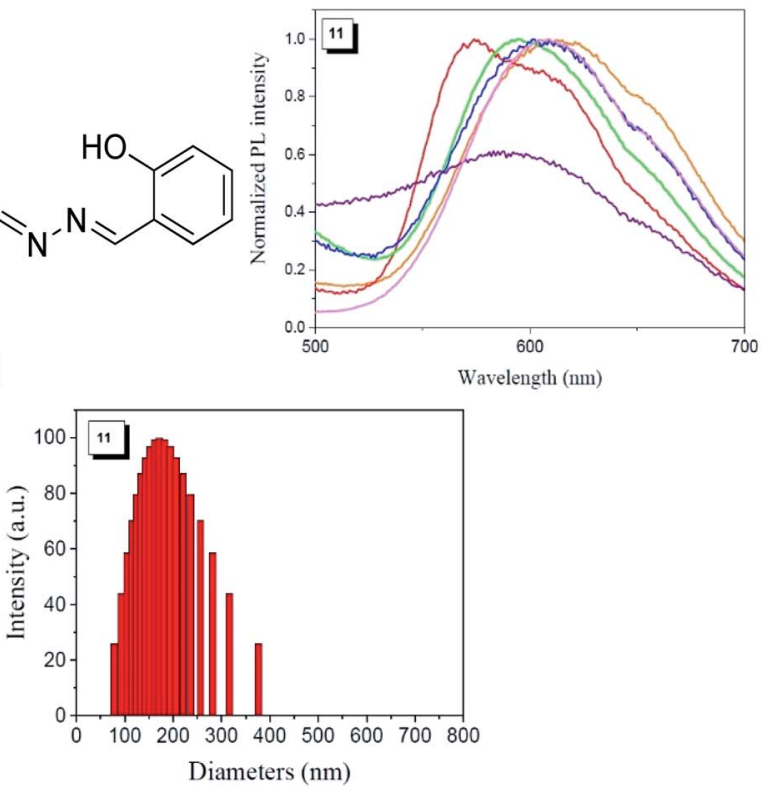

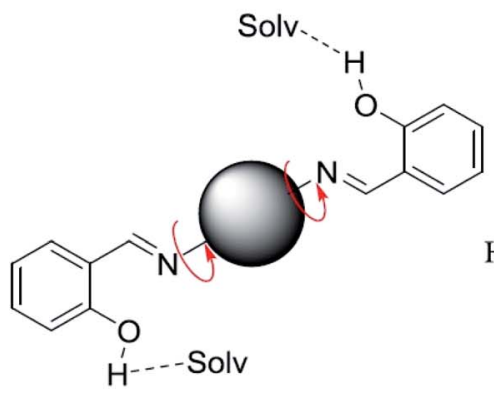

non-emissive single-molecule form

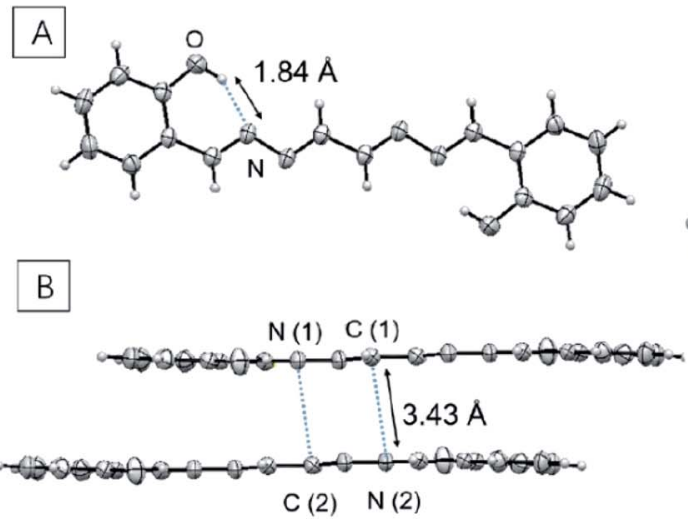

strong-emissive aggregation form

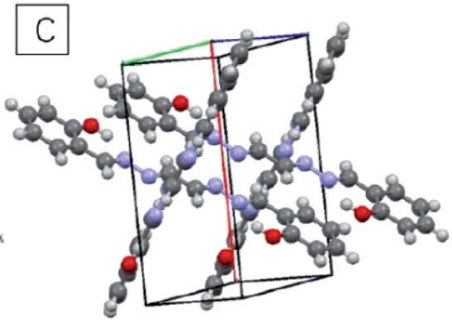

Fig. 20 (Top) Chemical structure, normalized PL spectra in different solvents, and distribution of the particle size of compound 15. (Middle) The proposed AIE mechanism of the SSB dye in aggregation form. Solv stands for solvent molecules. (Bottom) Single crystal structure of compound 15. (A) ORTEP drawing with $50 \%$ probability ellipsoids and hydrogen bond geometry; (B) side view and short contact geometry; (C) a unit cell. The figures were reproduced from ref. 112 with copyright permission from Elsevier.

wavelength and Stokes shift of more than $130 \mathrm{~nm}$, suggestive of an excimer emission when incorporated on the DNA backbone as a fluorescent label. ${ }^{\mathbf{1 2 1}}$ They showed an increase in the fluorescence quantum yield upon the addition of more than one fluorophore to encourage close interactions. This behavior has a perquisite when assessing for white light emission that comes from a mixture of red, green, and blue fluorophores. In general, when the number of monomers increased, the excimer-to- monomer ratio will markedly increase, and this feasibly enhances the fluorescence peak intensity. Kobori et al. also reported a remarkably large Stokes shift (230-330 nm) excimerforming candidate based on a cyanine dye and FRET-based pyrene probe. ${ }^{122}$ In their study, conjugation between the oligoribonucleotides and cyanine dye gave rise to a pyrene excimer in the excited state that contributed to the long-wavelength emission. Other research laboratories that worked on the 
pyrene series as an excimer emission characterized by a large Stokes shift extended the pyrene core with moieties like pyridinium and borane..$^{37,38,123}$

Luminescent alkali halide crystals exhibiting interesting photophysical and photochemical behaviors have been outlined by Li et al. using dicyanoaurate, dicyanoargentate and dicyanocuprate as the activator ions. ${ }^{\mathbf{1 2 4}}$ Closed-shell metals like gold can repel each other due to fully occupied valence orbitals, inducing a form of weak interaction called the aurophilic interaction. On this basis, they developed a ligand-unsupported $\mathrm{Ag}(\mathrm{I})$ complex, $\mathrm{T} 1\left[\mathrm{Ag}(\mathrm{CN})_{2}\right]$, that can form excimers $*\left[\mathrm{Ag}(\mathrm{CN})_{2}{ }^{-}\right]$ when in bulk condition. It was discovered that the bond distance between two $\mathrm{Ag}$ atoms was shorter in the excited state, while their binding energy and overlap population increased significantly in the same state. These resulted in a stable interaction between two monomeric units in the excited state (i.e., stable dimer). A kinetic study conducted suggested that there was a fast energy transfer between the $*\left[\mathrm{Ag}(\mathrm{CN})_{2}{ }^{-}\right]_{n}$ excimers and decrease in the HOMO-LUMO band gap, which promoted a longer wavelength emission and large Stokes shift. Similar manners were realized on $\mathrm{Au}(\mathrm{CN})_{2}{ }^{-}$and $\mathrm{Cu}(\mathrm{CN})_{2}{ }^{-}$, but each possessed different relativistic effects.

Hence, it is conspicuous that when screening for an organic molecular architecture for excimer emission, $\pi-\pi$ interactions are substantially essential within the chromophore. Extending the conjugation of the fused aromatic ring, therefore, is a rational design to enhance the number of non-covalent bonding interactions among the molecules. ${ }^{125}$ The concerns among researchers in the field are devoted on such systems that possess good solution processability, relatively good fluorescence quantum yield, good charge transport and other significant optoelectronic properties. As for inorganic approaches, continuous efforts toward varying the ligands on closed-shell metals with $\mathrm{d}^{10}$ electronic configuration have been done. ${ }^{\mathbf{1 2 6}}$ With the wide spectrum of metal donors that exist nowadays, it is anticipated that unique photo-induced dynamics in the excited state of both solid and solution forms will be prompted, especially long wavelength emission of more than $600 \mathrm{~nm}$ that comes with a large Stokes shift property and high emission intensity.

\subsection{Host-guest interaction}

White light emitters that originate from single molecules are promising due to their high color stability and tunability. In this section, we emphasize factors that inhibit the pathways of nonradiative relaxation as much as possible via supramolecular interaction. The said interaction focuses on the host-guest relationship between large molecules containing heavy atoms (Br, Cl, and others) and heteroatoms (O, S, N, and others). Hostguest molecules can generate the room-temperature phosphorescence (RTP) that is well known to display a long emissive wavelength, lifetime, and large Stokes shift. Ma et al. highlighted the importance of the host-guest assembling and supramolecular polymer system that manifested the described characteristics. ${ }^{127}$ Conventionally, the crystalline packing would be an efficient avenue to establish a rigid environment of the molecules for enhanced RTP, but non-crystalline materials also have very recently caught the attention of researchers. ${ }^{\mathbf{1 2 8 , 1 2 9}}$ These amorphous metal-free organic materials could potentially replace the rapid utilization of scarce and toxic metals in stride to develop large Stokes shift molecules.

$\beta$-Cyclodextrin ( $\beta$-CD) has been employed as a macrocycle host embedding the phosphor moieties as host-guest strategies. Several reports have been discussed about the unprecedented potentials of $\beta$-CD for having the hydrophobic cavity that can encapsulate guest molecules for RTP. ${ }^{92,130} \mathrm{Li}$ et al. synthesized different guest molecules to be incorporated onto the hydrophobic pockets of $\beta$-CD in order to attain multicolor emission via fluorescence-phosphorescence strategy. ${ }^{\mathbf{1 3 0}}$ In their study, four different phosphor materials were synthesized, 6bromo-2-naphthol (6-BrNp), 4-bromo-4'-hydroxybiphenyl (4BrHB), 4-(4-bromophenyl)-pyridine (4-BrBp) and 4-bromo-1,8naphthalic anhydride (4-BrNpA) (Fig. 21). The Stokes shifts of RTP emission for BrNp- $\beta$-CD and BrNpA- $\beta$-CD exceeded $230 \mathrm{~nm}$ and $220 \mathrm{~nm}$, respectively, while both BrHB- $\beta$-CD and 4-BrBp- $\beta$ $\mathrm{CD}$ reached over $200 \mathrm{~nm}$. All can be regarded as having large Stokes shifts. These compounds could be RTP emissive even in amorphous solid states, which makes them convenient for preparation and practical utility. The key approach to RTP emission with the presence of $\beta$-CD could be attributed to an external factor, such as suppression of non-radiative decay via increased of intermolecular hydrogen bonding atmosphere. Extending the guest molecule based on adamantine derivatives within the host formed a supramolecular complex that could be fine-tuned from yellow to purple emission, including white light.

In another work by Gong and his research group, cucurbit[7] uril, $(\mathrm{CB}[n])$ host was complexed with a 6-bromoisoquinoline derivative guest to form a pH-controlling molecular shuttle for intense RTP emission, which also gave an appreciably large difference in their excitation and emission wavelengths. ${ }^{\mathbf{1 3 1}}$ Similar to $\beta-\mathrm{CD}, \mathrm{CB}[n]$ is a barrel-shaped macrocycle host that bears a hydrophobic cavity to fabricate stable inclusion complexes with phosphor molecules. Since heavy atoms are capable of enhancing the intersystem crossing (ISC) rate of a chromophore, yielding a population of the triplet state and enhanced RTP emission, 6-bromoisoquinoline derivatives were selected as the guest molecules. ${ }^{\mathbf{1 3 2}}$ Experimental results revealed that a very stable host-guest system could be achieved in a nonacidic environment. The interesting feature of Gong's CB-RTP complex was the fact that upon illumination of UV light, the complex produced a considerably long emission wavelength near the green region, giving a Stokes shift value of more than $200 \mathrm{~nm}$ (Fig. 22). This emerged from the molecular switching of $\mathrm{CB}$ [7] via protonation or deprotonation of the terminal carboxyl group in water. An early article on the emission of isoquinoline derivatives showed a narrow to medium Stokes shift. ${ }^{133}$ Hence, it is evident that complexation with the host molecules unraveled a new route for future study that is to employ macrocyclic molecules to encapsulate "threads" of guest molecules, establishing a rigid environment in favor of better efficiency of the phosphorescence emission. However, a practical solution when employing host-guest recognition for white light in light- 
emitting diodes applications is to be investigated further. This is because most of the supramolecular entities have good solution processability and decent quantum efficiencies in polar solvents (i.e., water), but insufficient data have yet to be acquired to understand their behaviors in nonpolar media.

\subsection{Metal-ligand charge transfer (MLCT)}

For inorganic molecules, MLCT plays a crucial role in expressing a large Stokes shift value via phosphorescence by means of harvesting both singlet and triplet excitons. Therefore, many research groups have aimed the advancements of

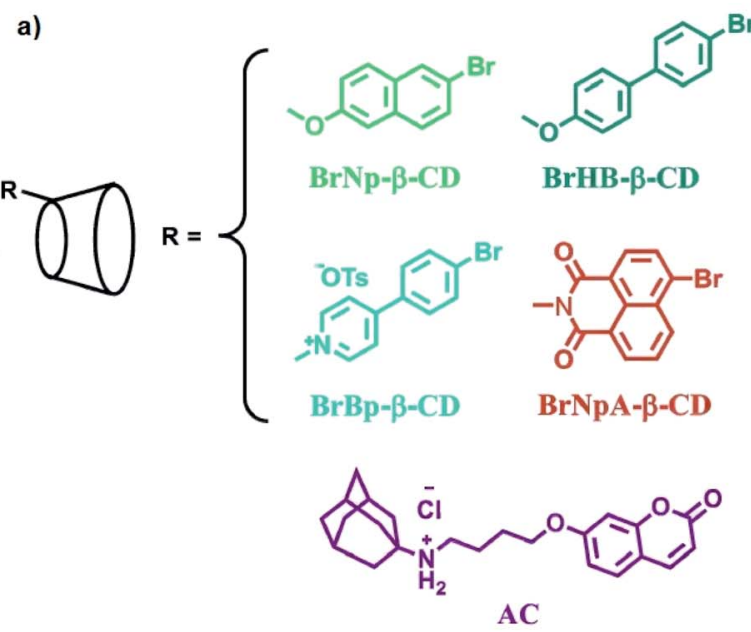

b)

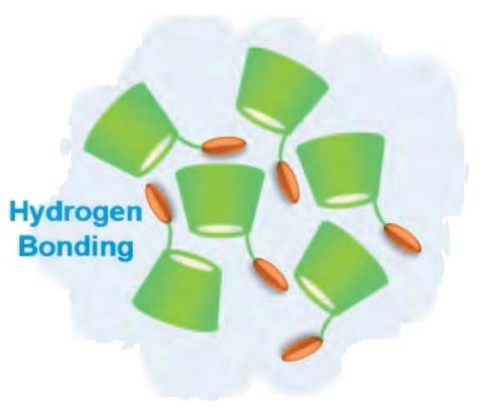

S
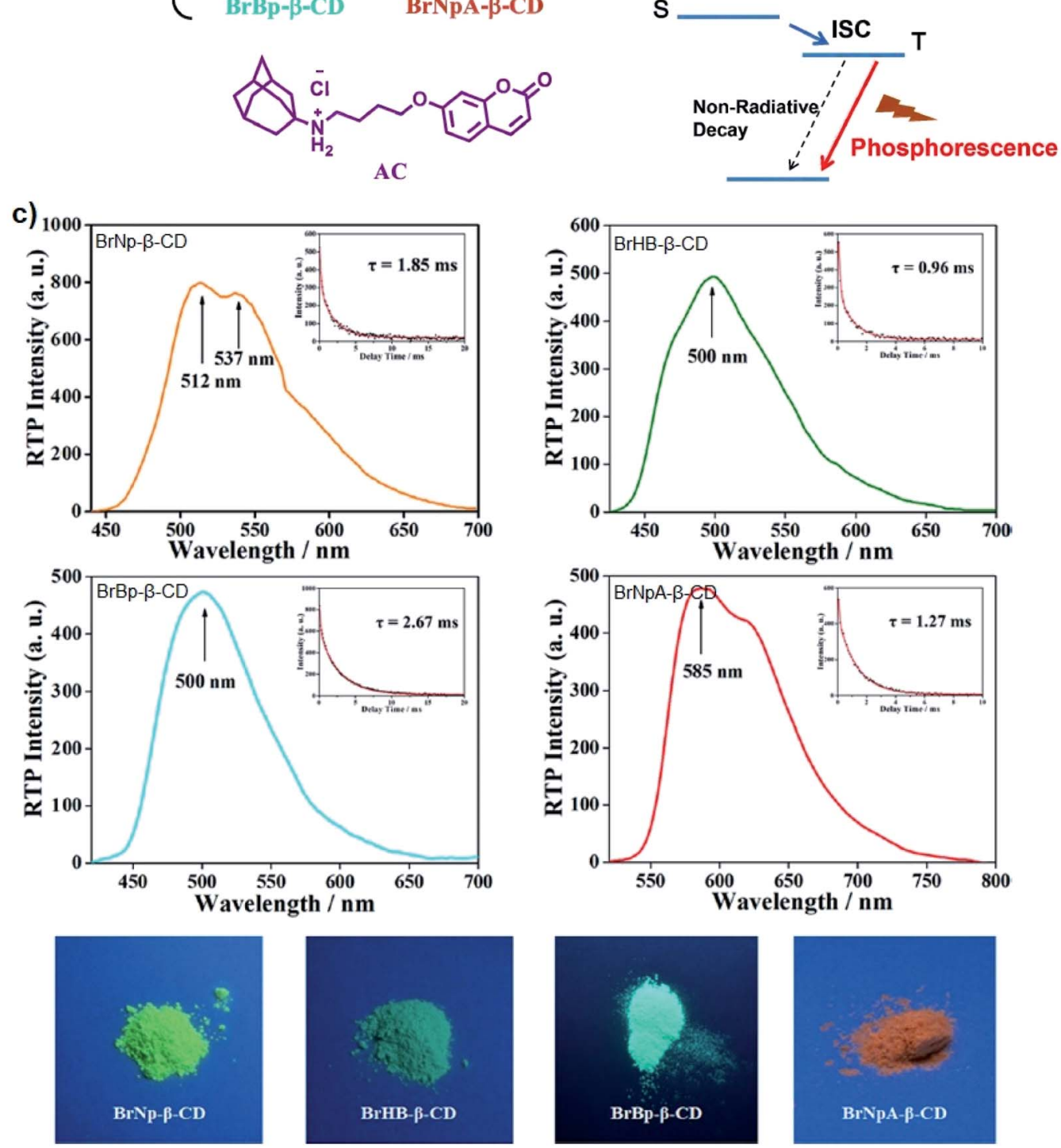

Fig. 21 (a) Molecular structures of RTP emissive cyclodextrin derivatives and the fluorescent guest molecule AC; (b) schematic representation of the phosphorescence emission; (c) RTP emission spectra in amorphous solid states with $\lambda_{\text {ex }}=285,300,350$, and 365 nm, respectively. Luminescent delay lifetime (insets) and photographs of the solid powder of the four RTP-emissive CD derivatives. The figures were adapted from ref. 130 with permission. Copyright 2018 American Chemical Society. 
light-emitting materials based on metals like iridium, platinum, osmium, rhenium, and copper. Li and co-workers have brought forward novel rhenium(I) complexes with large Stokes shift (16a and 16b) (Fig. 23). ${ }^{\mathbf{1 3 4}}$ Ligands based on decorated 1,10-phenanthroline were selected to bind to the central metal $\operatorname{Re}(\mathrm{I})$, corresponding to their bidentate chelating nature. The study demonstrated that both $\mathbf{1 6 a}$ and $\mathbf{1 6 b}$ were capable of displaying remarkably large Stokes shifts (>240 nm), which were attested to originate from MLCT processes. A deeper insight has shown that the absorption peaks of 16a, located at $287 \mathrm{~nm}$ and $342 \mathrm{~nm}$, were allocated to spin-allowed ${ }^{1} \pi-\pi^{*}$ ligand-centered electronic transitions. The said ligand in this compound 16a is 3,8-diphenyl-1,10-phenanthroline (PPhen). The extended absorption bands from $c a .380 \mathrm{~nm}$ to $550 \mathrm{~nm}$ were tentatively attributed to ${ }^{1} \mathrm{MLCT}$ and ${ }^{3} \mathrm{MLCT}$, respectively, implying movement of electrons from $\mathrm{d} \pi(\mathrm{Re}) \rightarrow \pi^{*}$ (ligand). Compound $\mathbf{1 6 b}$ possessed absorption band features similar to that of 16a, but with more intense MLCT bands. Emission wavelengths of both compounds inherently appeared from $c a$. $575 \mathrm{~nm}$ to $582 \mathrm{~nm}$, which proved the indispensability of the central metal to significantly red shift the photoluminescence spectra (from comparison with free ligand). The large Stokes shift also could be sourced from the structural changes that occur in the excited state upon delocalization of electrons between the metal and ligands that eventually relate back to the phenomenon of MLCT.

Copper complexes caught the heed of researchers very recently as well, owing to the intersystem crossing from singlet to triplet excited states in favor of high efficiency light emission. Zhang and co-workers surmounted the 25\% internal quantum efficiency limit in fluorescent materials by introducing $\mathrm{Cu}(\mathrm{I})$ complexes with different phosphine and phenanthroline ligands in the solid state. ${ }^{\mathbf{1 3 5}}$ The absorption maximum of the complexes ranged from 365-386 nm, attributed to the low-lying MLCT band, whereas the emission for different derivatives were $c a$. 504-555 $\mathrm{nm}$. These indefinitely opened a new path of large Stokes shift (>138 nm) materials based on the phosphorescence phenomenon of $\mathrm{Cu}$ (I) complexes, which are inexpensive and non-toxic to the environmental matrices. It is noteworthy to highlight that the presence of bulky alkyl ligands in positions 2 and 9 of the phenanthroline ligand led to blue-shifted emission. This was due to the sterically hindered structural deformation of the overall complex in the MLCT state, which narrowed the energy gap between the excited and ground states. Hence, care should be taken when screening for ligand candidates to bind to the central metal, whereby characteristics of rigidity and bulkiness need to be compromised. Another $\mathrm{Cu}(\mathrm{I})$ complex useful for a large Stokes shift has been reported by Singh and co-workers using 2-mercaptobenzimidazole as ligands. ${ }^{\mathbf{1 3 6}}$ Their compound was capable of depicting strong emission at $598 \mathrm{~nm}$ from an excitation of $300 \mathrm{~nm}$, giving a Stokes shift value of $298 \mathrm{~nm}$. Strong emission arose from MLCT when the 2-mercaptobenzimidazole ligands had more tendency to withdraw electrons from the copper(I) metal upon complex protonation in a solvent system of tetrahydrofuran : water.<smiles>Brc1ccc2cnccc2c1</smiles><smiles>CC(C)(C[n+]1ccc2cc(Br)ccc2c1)C(=O)O</smiles>
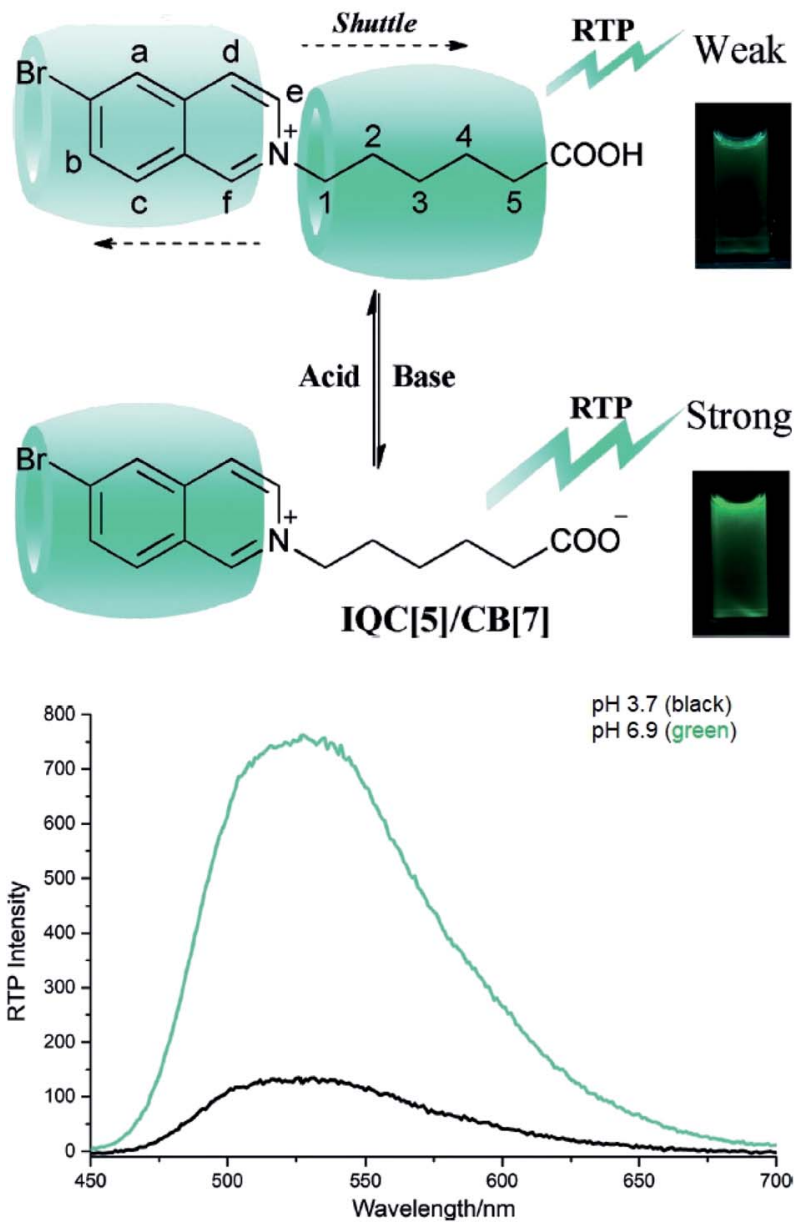

Fig. 22 Structures of the guests and the reversible inclusion phenomenon of the complex system. Photographs show the aqueous solution of the complex upon illumination with a handheld UV lamp $(254 \mathrm{~nm})$. Below shows the RTP spectrum of the host-guest system in water with $\lambda_{\mathrm{ex}}=300 \mathrm{~nm}$. The figures were reproduced from ref. 131 with copyright permission from John Wiley and Sons.

In 2019, AlAbbad and his group recently scrutinized the trans influence and substituent effects on the HOMO-LUMO energy gap and Stokes shift component in ruthenium mono-diimine derivatives. ${ }^{137}$ The push-pull effect in the context of the metal complex was sourced from the strong oxidant of the Ru metal. Electrons transfer from the $t_{2} g$ orbital of Ru to the low-lying $\pi^{*}$ molecular orbital of the 2,2'-bipyridyl ligand, invoking a singlet ground state $\left(\mathrm{S}_{0}\right)$ to singlet metal-to-ligand charge transfer $\left({ }^{1}\right.$ MLCT). Emission of the ${ }^{1}$ MLCT band for the Ru complex was known to produce a large Stokes shift $(\sim 150 \mathrm{~nm})$ and maximum wavelength reaching the NIR region of the spectrum. The longlived intensity decay, typically from $100 \mathrm{~ns}$ to $10 \mu \mathrm{s}$, was assigned to the triplet state ${ }^{3}$ MLCT emission. If we are to 
compare the emission wavelength of the $\mathrm{Ru}$ complexes designed by AlAbbad et al., it is apparent that the band redshifted for the Ru metal that accommodated stronger electron withdrawing ligands. Ru connected to the 4,4'-dicarboxylic-2,2' bipyridyl ligand had the longest emission wavelength at $639 \mathrm{~nm}$, followed by Ru linked to a bis- $4,4^{\prime}$-( $N$-methylamide)$2,2^{\prime}$-bipyridyl ligand at $582 \mathrm{~nm}$. The most blue-shifted emission of (514 nm) Ru complex held the ligand bis-4,4'-(methyl)-2,2'bipyridyl, which is the strongest electron donor group. From these examples, we have clearly depicted that the MLCT character can tune the Stokes shift value. Therefore, selecting a metal-ligand that can promote intersystem crossing to an excited triplet state can widen the gap between the absorption and emission bands.

\section{Classification of Stokes shifts by narrow, medium and large (mega)}

Since there were many reported fluorescent materials with varying components of Stokes shift, we would like to propose nuances or definite categories of the Stokes shifts so that future facile studies based on these characteristics can be referred by researchers when evaluating different types of molecules in specified fields of application. In this section, only selected organic compounds, including some that have been mentioned earlier in this review, will be listed since we could not accommodate all reported fluorescent compounds with varying backbone and skeletal system. The essence of the selected skeletal groups is well known to produce near infrared (NIR) emission that is especially important for many optoelectronic devices. NIR or red emission is highly demanding and ubiquitous for the generation of white lightemitting diodes.

Such selections are outlined to show that chemical modification of molecules and altering the chemical environment can substantially affect the photophysical properties and Stokes shift value of the compounds. With that, it is undeniably that for different skeletal groups, a continual and extensive investigation of this area requires further devotion in order to obtain thorough experimental and theoretical data for a more comprehensive referral. The skeletal groups selected are based on boron dipyrromethene (BODIPY), coumarin, cyanine, perylene, squaraine, and rhodamine molecules. Table 2 lists the categories of narrow, medium, and large (or termed as "mega" when it is exceptionally large) Stokes shifts based on the differences between the excitation and emission wavelength, and some examples of the chromophore. Table 3 tabulates the photophysical properties of the discussed NIR chromophores, including absorption and emission wavelength, fluorescence quantum yield and fluorescence lifetime.

There are other factors that could affect the Stokes shift value and emission shifting of compounds, including solvation dynamic, ${ }^{54,154} \mathrm{pH}$ effect, ${ }^{155}$ temperature, ${ }^{156,157}$ polymer dispersion, ${ }^{\mathbf{1 5 8}}$ and others. Therefore, the incorporation of different components of Stokes shift entails a joint venture of consideration between the molecular conformation and its nanoenvironments. By establishing the fine lines of Stokes shifts, we aspire for straightforward compound classification, in the endeavor of material screening for white light generation.
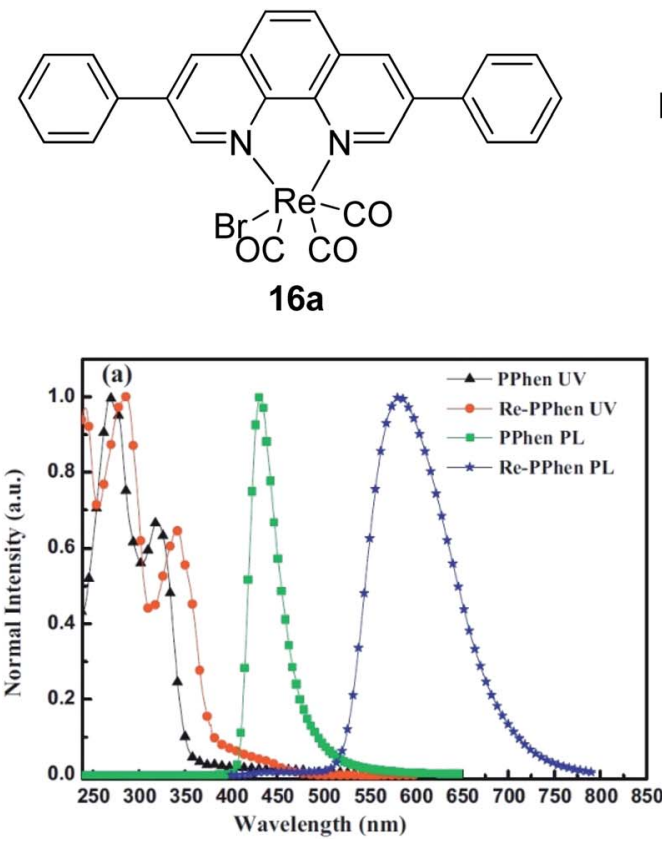
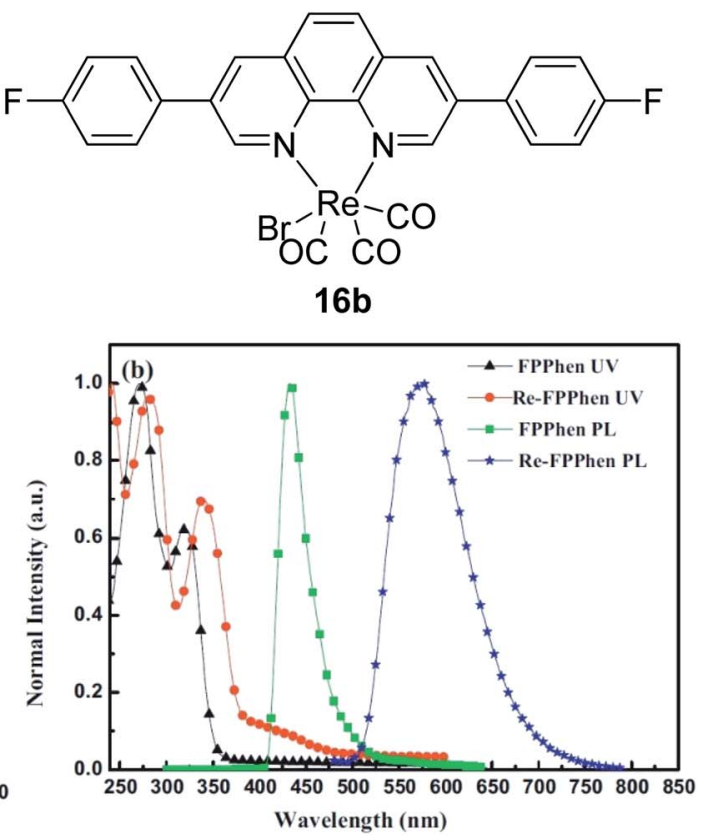

Fig. 23 Molecular structure of rhenium(I) complexes (16a and 16b), and their absorption and emission spectra in dichloromethane. (PPhen = 3,8diphenyl-1,10-phenanthroline; Re-PPhen = 16a; FPPhen = 3,8-bis(4-fluorophenyl)-1,10-phenanthroline; Re-FPPhen =16b). The figure was adapted from ref. 134 with copyright permission from Elsevier. 
Table 2 Category of Stokes shifts and examples. (DMF: dimethylformamide; $\mathrm{CH}_{2} \mathrm{Cl}_{2}$ : dichloromethane; EtOH: ethanol; $\mathrm{MeOH}$ : $\mathrm{methanol}$; PBS: phosphate-buffer saline; $\mathrm{CHCl}_{3}$ : chloroform; PhMe: toluene; DMSO: dimethyl sulfoxide)

Narrow Stokes shift, $\Delta \lambda \leq 50 \mathrm{~nm}$<smiles></smiles><smiles></smiles>

$17 \mathrm{a}$ $\Delta \Lambda(17 a)=10 \mathrm{~nm}\left(\right.$ in $\left.\mathrm{CH}_{2} \mathrm{Cl}_{2}\right)$
Medium Stokes shift, $50 \mathrm{~nm}$ $<\Delta \lambda<100 \mathrm{~nm}$<smiles></smiles><smiles></smiles>

$17 b$ $\Delta \Lambda(17 b)=67 \mathrm{~nm}\left(\right.$ in $\left.\mathrm{CH}_{2} \mathrm{Cl}_{2}\right)$
Large or mega Stokes shift, $\Delta \lambda \geq$ $100 \mathrm{~nm}$

References

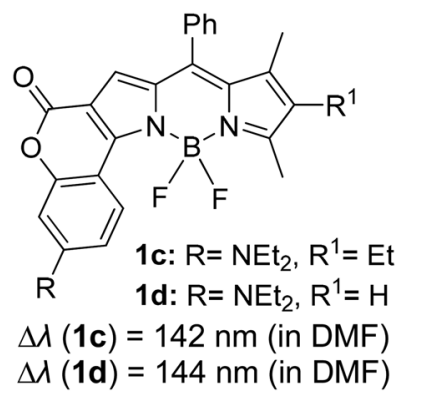

40

138 and 139<smiles>Cc1[nH]c2ccc3oc(=O)cc(-c4ccc(C#N)cc4)c3c2c1C</smiles><smiles>Cc1[nH]c2ccc3oc(=O)c(-c4ccc(N(C)C)cc4)cc3c2c1C</smiles><smiles>Cc1[nH]c2cc3c(-c4ccc(C#N)cc4)cc(=O)oc3cc2c1C</smiles>

$\Delta \Lambda(2 a)=159 \mathrm{~nm}\left(\right.$ in $\left.\mathrm{CH}_{2} \mathrm{Cl}_{2}\right)$

$\Delta \lambda(\mathbf{2 b})=113 \mathrm{~nm}\left(\right.$ in $\left.\mathrm{CH}_{2} \mathrm{Cl}_{2}\right)$

$\Delta \Lambda(2 c)=191 \mathrm{~nm}\left(\right.$ in $\left.\mathrm{CH}_{2} \mathrm{Cl}_{2}\right)$<smiles></smiles>

$\Delta \Lambda(18 c)=77 \mathrm{~nm}($ in $\mathrm{EtOH})$ 
Table 2 (Contd.)

Medium Stokes shift, $50 \mathrm{~nm}$ $<\Delta \lambda<100 \mathrm{~nm}$<smiles>COC(=O)c1cc2ccc(N)cc2oc1=O</smiles>

$18 \mathrm{a}$

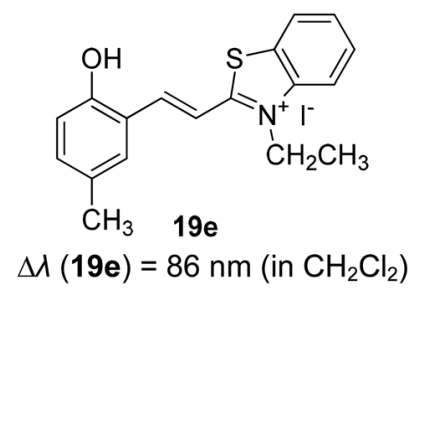<smiles>CCCCCCCCC(C)(C)C</smiles>

$\Delta \Lambda(18 a)=43 \mathrm{~nm}($ in $\mathrm{EtOH})$

$\Delta \Lambda(\mathbf{1 8 b})=41 \mathrm{~nm}($ in $\mathrm{EtOH})$

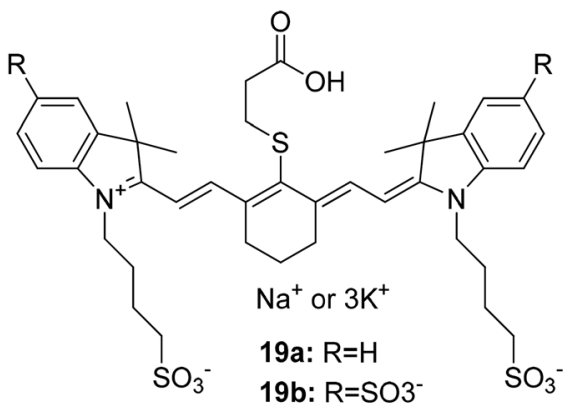

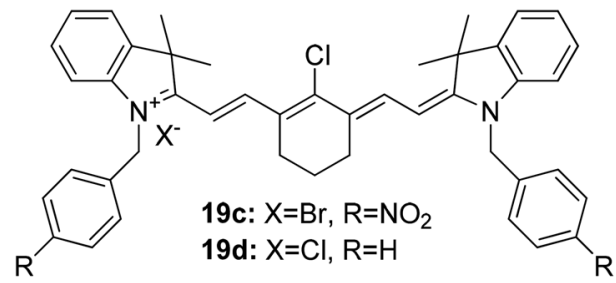

$\Delta \Lambda(19 a)=35 \mathrm{~nm}$ (in PBS) $\Delta \Lambda(19 b)=31 \mathrm{~nm}$ (in PBS) $\Delta \Lambda(19 \mathrm{c})=22 \mathrm{~nm}($ in $\mathrm{MeOH})$ $\Delta \lambda(19 d)=29 \mathrm{~nm}($ in $\mathrm{MeOH})$<smiles>CC1(C)C(/C=C/C2=C(Cl)/C(=C/C=C3\N(Cc4ccccc4)c4ccc(S(=O)(=O)O)cc4C3(C)C)CCC2)=[N+](Cc2ccccc2)c2ccc(S(=O)(=O)O)cc21</smiles>
$\Delta \lambda(\mathbf{2 0 a})=20 \mathrm{~nm}($ in $\mathrm{MeOH})$
Large or mega Stokes shift, $\Delta \lambda \geq$ $100 \mathrm{~nm}$

References

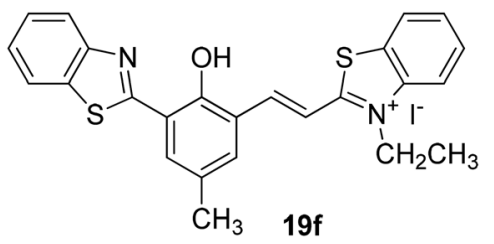

$\Delta \Lambda(\mathbf{1 8 e})=120 \mathrm{~nm}$ (in $\mathrm{EtOH})$

$$
\Delta \lambda(19 f)=234 \mathrm{~nm}\left(\text { in } \mathrm{CH}_{2} \mathrm{Cl}_{2}\right)
$$


Table 2 (Contd.)

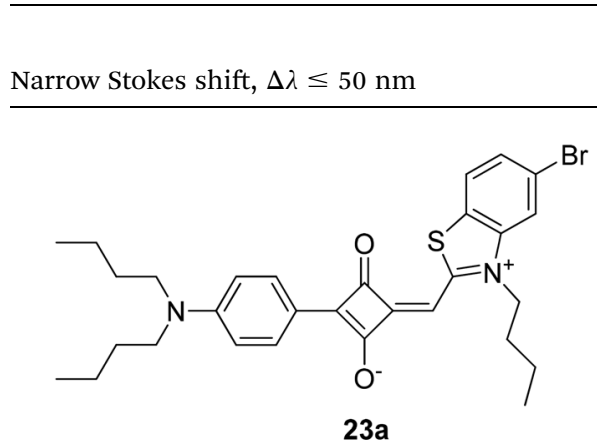

23a

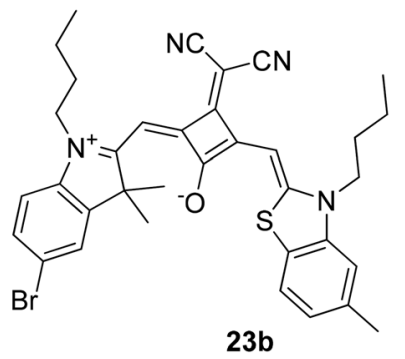

$\Delta \lambda(23 a)=35 \mathrm{~nm}($ in DMSO $)$

$\Delta(\mathbf{2 3 b})=32 \mathrm{~nm}$ (in DMSO)<smiles>[R]c1cc2c(-c3ccccc3C(=O)O)c3cc([R])c(=[N+]([R])[R])cc-3oc2cc1N([R])[R]</smiles><smiles>[R]C1=CC2=C(c3ccccc3C(=O)O)c3cc([R])c(N([R])[R])cc3[Si](C)(C)C2=CC1=[N+]([R])[R]</smiles>

24b

$\Delta \Lambda(\mathbf{2 4 a})=20 \mathrm{~nm}($ in $\mathrm{EtOH})$

$\Delta \Lambda(24 b)=18 \mathrm{~nm}($ in $\mathrm{EtOH})$<smiles>CCN(CC)c1ccc2c(c1)Oc1c(cc3c4c1CCCN4CCC3)C21OC(=O)c2ccccc21</smiles>

$25 a$

$\Delta \lambda(25 a)=27 \mathrm{~nm}($ in PhMe $)$
Medium Stokes shift, $50 \mathrm{~nm}$ $<\Delta \lambda<100 \mathrm{~nm}$
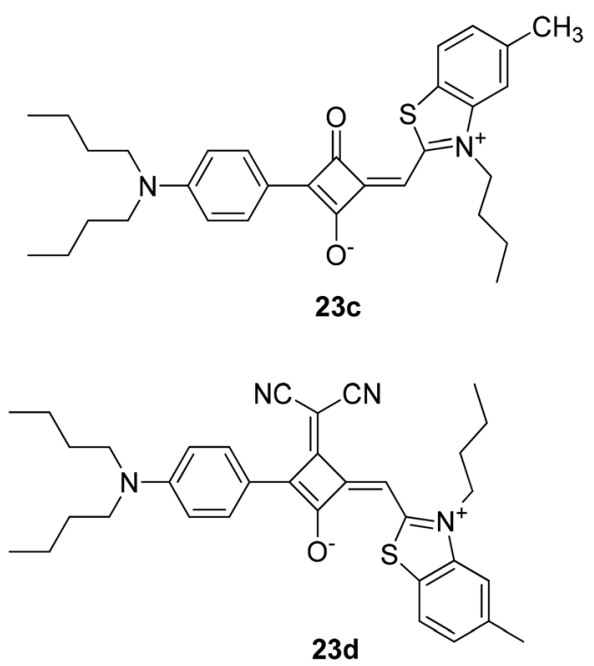

$\Delta \lambda($ 23c $)=57 \mathrm{~nm}($ in DMSO) $\Delta \lambda(\mathbf{2 3 d})=90 \mathrm{~nm}($ in DMSO)

146<smiles>CCN(CC)c1ccc2c(c1)Oc1cc3c(cc1=C2c1ccccc1C(=O)O)Sc1ccccc1[N+]=3CC</smiles>

$\Delta \Lambda(\mathbf{2 4 c})=124 \mathrm{~nm}($ in $\mathrm{EtOH})$ 
Table 2 (Contd.)

Medium Stokes shift, $50 \mathrm{~nm}$ $<\Delta \lambda<100 \mathrm{~nm}$
Large or mega Stokes shift, $\Delta \lambda \geq$ $100 \mathrm{~nm}$ References<smiles>[R]c1ccc2cc(-c3ccc(-c4c5ccc(=[N+]([C-]C)CC)cc-5oc5cc(N(CC)CC)ccc45)cc3)c(=O)oc2c1</smiles><smiles>CCN(CC)c1ccc2c(-c3ccc(-c4cc5c(ccc6ccccc65)oc4=O)cc3)c3ccc(=[N+](CC)CC)cc-3oc2c1</smiles>

$\triangle \Lambda(\mathbf{2 6 d})=229 \mathrm{~nm}$ (in PBS $/ \mathrm{MeOH})$

$\Delta \lambda(\mathbf{2 6 e})=231 \mathrm{~nm}($ in $\mathrm{PBS} / \mathrm{MeOH})$

$\Delta \lambda(\mathbf{2 6 f})=228 \mathrm{~nm}($ in $\mathrm{PBS} / \mathrm{MeOH})$

$\Delta \lambda(\mathbf{2 6 g})=210 \mathrm{~nm}($ in $\mathrm{PBS} / \mathrm{MeOH})$

\section{Conclusion and perspectives}

White light emission has been arousing great interests among researchers of different backgrounds, and plenty of research works have been accomplished to achieve emitters exhibiting large or mega Stokes shift property. Instead of discussing the plethora of methods in generating white light, we provided a focused discussion on materials designation. This review article has highlighted some recent developments of white light emitters that employ large or mega Stokes shift molecules both from organic and inorganic sources. The hallmark of large or mega Stokes shift is the fine control over the emission color through a multi-fluorophore system, mainly from red, green, and blue (RGB) emitters. This could provide a promising avenue to future optoelectronic devices and lighting applications that would unveil high color rendition, more energy efficiency, enhanced photostability and quality, and ease of device fabrication. Thus, it is necessary to understand the photophysical mechanisms that engender large or mega Stokes shift molecule. The processes that have been covered include ESIPT, ICT, excited state geometry relaxation or structure deformation, AIE, concessions between monomer and excimer, host-guest interaction and MLCT.

However, further devotion needs to be given in order to obtain large or mega Stokes shift materials with high quantum efficiency. Most of the previously reported low energy or red region emitters have considerably low fluorescence intensity due to the frantic, undesirable non-radiative decay pathways that occur extremely rapidly $\left(10^{-12}\right.$ to $\left.10^{-10} \mathrm{~s}\right)$. In view of this, both experimental and simulation (theoretical) data of fluorescent materials from different backbones need to be extended 
for a deeper understanding of the electronic transitions that take place within the molecule, i.e., intramolecular and intermolecular interactions based on the molecule functionalities and the applied chemical environments. The effort to harvest triplet excitons by thermally activated delayed fluorescence (TADF) is one of the progressing strategies for ameliorated

Table 3 Photoluminescence property variations for NIR molecules

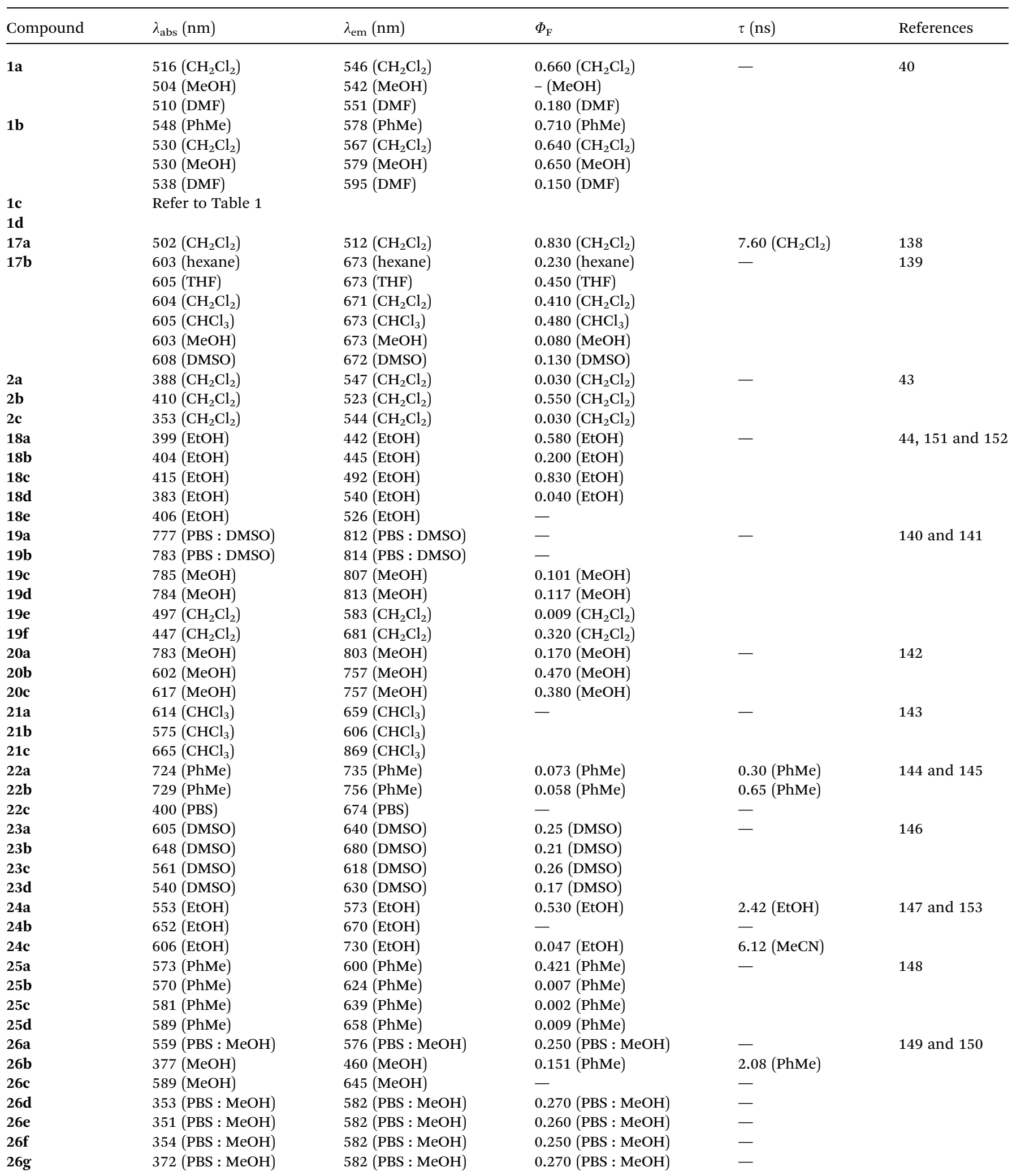


quantum yield emission. Nonetheless, it comes with a competing effect that is the small gap (normally $<0.2 \mathrm{eV}$ ) between the absorbed and emitted photons. Hence, it could be appended as a judicious approach in achieving both large or mega Stokes shift and superior fluorescence intensity. The architecture of the molecule possessing both properties that are sought after will therefore be a non-trivial task. It will especially be an arduous journey to optimize the contrasting properties to generate a pure and stable white light. To summarize, the common approaches aiming for a large or mega Stokes shift molecule are to construct a systematic material design, carrying an energy-donor-acceptor framework or/and extending the $\pi$ conjugation length of the compound. These are due to the elusive nature of the molecule's excited state upon photoexcitation. The aforementioned subtopics that revolve around the white light and Stokes shifts are in fact fascinating to further gain in-depth insight, and thus related prospects could evolve and flourish in the near future in the field of display devices, artificial lighting, bioimaging, and photosensitisers.

\section{Author contributions}

Nadia Nabihah Mohd Yusof Chan writing - original draft, investigation, formal analysis. Azila Idris writing - review \& editing, supervision, funding acquisition. Zul Hazrin Zainal Abidin supervision, validation. Hairul Anuar Tajuddin supervision, conceptualization. Zanariah Abdullah validation, project administration, resources.

\section{Conflicts of interest}

The authors declare no conflicts of interest.

\section{Acknowledgements}

The authors would like to express gratitude to the Ministry of Higher Education (MOHE) Malaysia for providing the Fundamental Research Grant Scheme (FP028-2019A).

\section{References}

1 S. Muthu, F. J. Schuurmans and M. D. Pashley, Red, green, and blue LED based white light generation: issues and control, Conference Record of the IEEE Industry Applications Conference, IEEE Publisher, 2002, pp. 327-333, DOI: 10.1109/IAS.2002.1044108.

2 J. M. Stone and J. C. Knight, Opt. Express, 2008, 16, 26702675.

3 A. Aguilar-Castillo, J. Aguilar-Hernández, M. GarcíaHipólito, S. López-Romero, R. Swarnkar, A. BáezRodríguez, R. Fragoso-Soriano and C. Falcony, Ceram. Int., 2017, 43, 355-362.

4 J. S. Kim, P. E. Jeon, Y. H. Park, J. C. Choi, H. L. Park, G. C. Kim and T. W. Kim, Appl. Phys. Lett., 2004, 85, 36963698.

5 I. Isnaeni and I. T. Sugiarto, ITB J. Math. Fundam. Sci., 2017, 49, 57-65.
6 G. M. Farinola and R. Ragni, Chem. Soc. Rev., 2011, 40, 3467-3482.

7 J. Gotta, T. B. Shalom, S. Aslanoglou, A. Cifuentes-Rius, N. H. Voelcker, R. Elnathan, O. Shoseyov and S. Richter, Adv. Funct. Mater., 2018, 1706967.

8 J. Wang, X. Gu, H. Ma, Q. Peng, X. Huang, X. Zheng, S. H. Sung, G. Shan, J. W. Lam and Z. Shuai, Nat. Commun., 2018, 9, 1-9.

9 N. M. Sarih, P. Myers, A. Slater, B. Slater, Z. Abdullah, H. A. Tajuddin and S. Maher, Sci. Rep., 2019, 9, 1-8.

10 S. Tanwar, B. Sharma, V. Kaur and T. Sen, RSC Adv., 2019, 9, 15997-16006.

11 R. Wang, J. Peng, F. Qiu, Y. Yang and Z. Xie, Chem. Commun., 2009, 6723-6725.

12 J. Zhao, S. Ji, Y. Chen, H. Guo and P. Yang, Phys. Chem. Chem. Phys., 2012, 14, 8803-8817.

13 X. Feng, F. Zhang, Y. Wang, Y. Zhang, Y. Yang and X. Liu, Appl. Phys. Lett., 2015, 107, 213102.

14 B. Chen, A. S. Susha, C. J. Reckmeier, S. V. Kershaw, Y. Wang, B. Zou, H. Zhong and A. L. Rogach, Adv. Mater., 2017, 29, 1604284.

15 W.-C. Geng, Y.-C. Liu, Y.-Y. Wang, Z. Xu, Z. Zheng, C.-B. Yang and D.-S. Guo, Chem. Commun., 2017, 53, 392-395.

16 E. R. Dohner, E. T. Hoke and H. I. Karunadasa, J. Am. Chem. Soc., 2014, 136, 1718-1721.

17 Y. Kubo and R. Nishiyabu, Polymer, 2017, 128, 257-275.

18 M. Bälter, S. Li, M. Morimoto, S. Tang, J. Hernando, G. Guirado, M. Irie, F. M. Raymo and J. Andréasson, Chem. Sci., 2016, 7, 5867-5871.

19 R. Wang, J. Peng, F. Qiu and Y. Yang, Chem. Commun., 2011, 47, 2787-2789.

20 R.-J. Xie, N. Hirosaki, T. Suehiro, F.-F. Xu and M. Mitomo, Chem. Mater., 2006, 18, 5578-5583.

21 Z. Wang, F. Yuan, X. Li, Y. Li, H. Zhong, L. Fan and S. Yang, Adv. Mater., 2017, 29, 1702910.

22 B. Yuan, S. Guan, X. Sun, X. Li, H. Zeng, Z. Xie, P. Chen and S. Zhou, ACS Appl. Mater. Interfaces, 2018, 10, 16005-16014.

23 S. Mandani, B. Sharma, D. Dey and T. K. Sarma, RSC Adv., 2016, 6, 84599-84603.

24 M. C. Gather, A. Köhnen and K. Meerholz, Adv. Mater., 2011, 23, 233-248.

25 J. E. Agudo, P. J. Pardo, H. Sánchez, Á. L. Pérez and M. I. Suero, Sensors, 2014, 14, 11943-11956.

26 S. Mukherjee and P. Thilagar, Dyes Pigm., 2014, 110, 2-27.

27 J. R. Lakowicz, Principles of fluorescence spectroscopy, Springer Science \& Business Media, 2013.

28 P. Wen, Z. Gao, R. Zhang, A. Li, F. Zhang, J. Li, J. Xie, Y. Wu, M. Wu and K. Guo, J. Mater. Chem. C, 2017, 5, 6136-6143.

29 D. R. Mohbiya and N. Sekar, ChemistrySelect, 2018, 3, 16351644.

30 I. d. L. de Sousa, V. F. Ximenes, A. R. de Souza and N. H. Morgon, J. Mol. Struct., 2019, 1192, 186-191.

31 B. Czaplinska, A. Maron, J. G. Malecki, G. Szafraniec-Gorol, M. Matussek, K. Malarz, A. Mrozek-Wilczkiewicz, W. Danikiewicz, R. Musiol and A. Slodek, Dyes Pigm., 2017, 144, 119-132. 
32 M. H. Stopel, C. Blum and V. Subramaniam, J. Phys. Chem. Lett., 2014, 5, 3259-3264.

33 Z. Yang, C. Yan, Y. Chen, C. Zhu, C. Zhang, X. Dong, W. Yang, Z. Guo, Y. Lu and W. He, Dalton Trans., 2011, 40, 2173-2176.

34 P. Song, S.-G. Sun, J.-Y. Liu, Y.-Q. Xu, K.-L. Han and X.-J. Peng, Spectrochim. Acta, Part A, 2009, 74, 753-757.

35 K. Fan, X. Wang, Y. Ma, H. Yang, G. Han, L. Zhou and S. Fang, New J. Chem., 2020, 44, 8351-8356.

36 P. Liu, G. Shi and X. Chen, Front. Chem., 2020, 8, 923.

37 C. S. Abeywickrama, K. J. Wijesinghe, R. V. Stahelin and Y. Pang, Chem. Commun., 2017, 53, 5886-5889.

38 Q. Chen, K. Cheng, W. Wang, L. Yang, Y. Xie, L. Feng, J. Zhang, H. Zhang and H. Sun, J. Pharm. Anal., 2020, 10, 490-497.

39 J. Chao, K. Song, Y. Zhang, C. Yin, F. Huo, J. Wang and T. Zhang, Talanta, 2018, 189, 150-156.

40 A. Y. Bochkov, I. O. Akchurin, O. A. Dyachenko and V. F. Traven, Chem. Commun., 2013, 49, 11653-11655.

41 F. Z. Li, L. L. Zhou and G. C. Kuang, Chem.-Asian J., 2021, 16, 97-101.

42 M. V. Sednev, V. N. Belov and S. W. Hell, Methods Appl. Fluoresc., 2015, 3, 042004.

43 L. Chen, T. S. Hu and Z. J. Yao, Eur. J. Org. Chem., 2008, 2008, 6175-6182.

44 X. Liu, Z. Xu and J. M. Cole, J. Phys. Chem. C, 2013, 117, 16584-16595.

45 X. Li, Y. Han, K. Min and Y.-A. Son, Mol. Cryst. Liq. Cryst., 2018, 660, 10-16.

46 W.-H. Jin, H.-H. Lu, Q. Zhang and D.-H. Qu, Mater. Chem. Front., 2020, 4, 532-536.

47 B. Gu, L. Huang, W. Su, X. Duan, H. Li and S. Yao, Anal. Chim. Acta, 2017, 954, 97-104.

48 Z. Gao, Y. Hao, M. Zheng and Y. Chen, RSC Adv., 2017, 7, 7604-7609.

49 Y. He, Q. Bing, Y. Wei, H. Zhang and G. Wang, Luminescence, 2019, 34, 153-161.

50 Y.-H. Zhao, Y. Luo, H. Wang, H. Wei, T. Guo, H. Tan, L. Yuan and X.-B. Zhang, Anal. Chim. Acta, 2019, 1065, 134-141.

51 L. Xie, Y. Chen, W. Wu, H. Guo, J. Zhao and X. Yu, Dyes Pigm., 2012, 92, 1361-1369.

52 B. Czaplińska, K. Malarz, A. Mrozek-Wilczkiewicz, A. Slodek, M. Korzec and R. Musiol, Molecules, 2020, 25, 2488.

53 C. Santana-Calvo, F. Romero, I. López-González and T. Nishigaki, BioTechniques, 2018, 65, 211-218.

54 S. Hisham, H. A. Tajuddin, C. C. Fei, Z. A. Hasan and Z. Abdullah, J. Lumin., 2018, 208(2019), 245-252.

55 B. Sk, S. Khodia and A. Patra, Chem. Commun., 2018, 54, 1786-1789.

56 L. Xu, Y. Zhao, G. Long, Y. Wang, J. Zhao, D. Li, J. Li, R. Ganguly, Y. Li and H. Sun, RSC Adv., 2015, 5, 6308063086.

57 G. Volpi, G. Magnano, I. Benesperi, D. Saccone, E. Priola, V. Gianotti, M. Milanesio, E. Conterosito, C. Barolo and G. Viscardi, Dyes Pigm., 2017, 137, 152-164.
58 M. J. Percino, M. Cerón, P. Venkatesan, E. Pérez-Gutiérrez, P. Santos, P. Ceballos, A. E. Castillo, P. Gordillo-Guerra, K. Anandhan and O. Barbosa-García, RSC Adv., 2019, 9, 28704-28717.

59 J. Jiang, Z. Xu, J. Zhou, M. Hanif, Q. Jiang, D. Hu, R. Zhao, C. Wang, L. Liu and D. Ma, Chem. Mater., 2019, 31, 6499-6505.

60 M. Chen, Y. Zhao, Z. Tang, B. Zhang and B. Wei, Chem. Rec., 2019, 19, 1768-1778.

61 C. Li, W. Chen, D. Wu, D. Quan, Z. Zhou, J. Hao, J. Qin, Y. Li, Z. He and K. Wang, Sci. Rep., 2015, 5, 17777.

62 H. Zhao, G. Liu, S. You, F. V. Camargo, M. Zavelani-Rossi, X. Wang, C. Sun, B. Liu, Y. Zhang and G. Han, Energy Environ. Sci., 2021, 14, 396-406.

63 F. Khan and J. H. Kim, ACS Photonics, 2018, 5, 4637-4643. 64 Y. Shishino, T. Yonezawa, K. Kawai and H. Nishihara, Chem. Commun., 2010, 46, 7211-7213.

65 A. Wen, X. Peng, P. Zhang, Y. Long, H. Gong, Q. Xie, M. Yue and S. Chen, Anal. Bioanal. Chem., 2018, 410, 6489-6495.

66 C. Zhou, H. Lin, H. Shi, Y. Tian, C. Pak, M. Shatruk, Y. Zhou, P. Djurovich, M. H. Du and B. Ma, Angew. Chem., Int. Ed., 2018, 57, 1021-1024.

67 L. Y. Liu, H. Fang, Q. Chen, M. H. Y. Chan, M. Ng, K. N. Wang, W. Liu, Z. Tian, J. Diao and Z. W. Mao, Angew. Chem., 2020, 132, 19391-19398.

68 Y. Zhang, B. Fan, Y. Liu, H. Li, K. Deng and J. Fan, Appl. Phys. Lett., 2018, 112, 183101.

69 S. Li, J. Luo, J. Liu and J. Tang, J. Phys. Chem. Lett., 2019, 10, 1999-2007.

70 X. Zhang, H.-C. Wang, A.-C. Tang, S.-Y. Lin, H.-C. Tong, C.-Y. Chen, Y.-C. Lee, T.-L. Tsai and R.-S. Liu, Chem. Mater., 2016, 28, 8493-8497.

71 V. S. Padalkar and S. Seki, Chem. Soc. Rev., 2016, 45, 169202.

72 J. Cheng, D. Liu, L. Bao, K. Xu, Y. Yang and K. Han, Chem.Asian J., 2014, 9, 3215-3220.

73 A. C. Sedgwick, L. Wu, H.-H. Han, S. D. Bull, X.-P. He, T. D. James, J. L. Sessler, B. Z. Tang, H. Tian and J. Yoon, Chem. Soc. Rev., 2018, 47, 8842-8880.

74 T. Iijima, A. Momotake, Y. Shinohara, T. Sato, Y. Nishimura and T. Arai, J. Phys. Chem. A, 2010, 114, 1603-1609.

75 V. S. Padalkar, A. Tathe, V. D. Gupta, V. S. Patil, K. Phatangare and N. Sekar, J. Fluoresc., 2012, 22, 311-322.

76 V. S. Patil, V. S. Padalkar, A. B. Tathe, V. D. Gupta and N. Sekar, J. Fluoresc., 2013, 23, 1019-1029.

77 H. Ren, F. Huo, Y. Zhang, S. Zhao and C. Yin, Sens. Actuators, B, 2020, 319, 128248.

78 Z. Zhang, Y.-A. Chen, W.-Y. Hung, W.-F. Tang, Y.-H. Hsu, C.-L. Chen, F.-Y. Meng and P.-T. Chou, Chem. Mater., 2016, 28, 8815-8824.

79 S. B. Yadav, S. Kothavale and N. Sekar, J. Mol. Liq., 2019, 294, 111626.

80 S. Barman, S. K. Mukhopadhyay, M. Gangopadhyay, S. Biswas, S. Dey and N. P. Singh, J. Mater. Chem. B, 2015, 3, 3490-3497.

81 H.-W. Tseng, T.-C. Lin, C.-L. Chen, T.-C. Lin, Y.-A. Chen, J.-Q. Liu, C.-H. Hung, C.-M. Chao, K.-M. Liu and P.-T. Chou, Chem. Commun., 2015, 51, 16099-16102. 
82 H. Okamoto, K. Itani, M. Yamaji, H. Konishi and H. Ota, Tetrahedron Lett., 2018, 59, 388-391.

83 Y. Zhang, J.-H. Wang, W. Zheng, T. Chen, Q.-X. Tong and D. Li, J. Mater. Chem. B, 2014, 2, 4159-4166.

84 T. Mutai, H. Sawatani, T. Shida, H. Shono and K. Araki, J. Org. Chem., 2013, 78, 2482-2489.

85 M. Yang, I. S. Park, Y. Miyashita, K. Tanaka and T. Yasuda, Angew. Chem., Int. Ed., 2020, 13955-13961.

86 F. Bureš, RSC Adv., 2014, 4, 58826-58851.

87 H. Tanaka, K. Shizu, H. Nakanotani and C. Adachi, J. Phys. Chem. C, 2014, 118, 15985-15994.

88 Y. Zhan, J. Zhao, P. Yang and W. Ye, RSC Adv., 2016, 6, 92144-92151.

89 A. Slodek, D. Zych, A. Maroń, S. Golba, E. Schab-Balcerzak, H. Janeczek, M. Siwy and S. Maćkowski, Dyes Pigm., 2019, 166, 98-106.

90 S. B. Yadav, S. Kothavale and N. Sekar, J. Photochem. Photobiol., A, 2019, 382, 111937.

91 M. Zhu, Y. Xu, L. Sang, Z. Zhao, L. Wang, X. Wu, F. Fan, Y. Wang and H. Li, Environ. Pollut., 2020, 256, 113427.

92 D. Li, W. Hu, J. Wang, Q. Zhang, X.-M. Cao, X. Ma and H. Tian, Chem. Sci., 2018, 9, 5709-5715.

93 A. Gandioso, R. Bresolí-Obach, A. Nin-Hill, M. Bosch, M. Palau, A. Galindo, S. Contreras, A. Rovira, C. Rovira and S. Nonell, J. Org. Chem., 2018, 83, 1185-1195.

94 Z. R. Grabowski, K. Rotkiewicz and W. Rettig, Chem. Rev., 2003, 103, 3899-4032.

95 Z. Zhang, G. Zhang, J. Wang, S. Sun and Z. Zhang, Comput. Theor. Chem., 2016, 1095, 44-53.

96 S. K. Panja, N. Dwivedi and S. Saha, $R S C A d v ., 2016,6$, 105786-105794.

97 A. Slodek, M. Filapek, G. Szafraniec, I. Grudzka, W. A. Pisarski, J. G. Malecki, L. Zur, M. Grela, W. Danikiewicz and S. Krompiec, Eur. J. Org. Chem., 2014, 2014, 5256-5264.

98 Y. Chen, J. Zhao, H. Guo and L. Xie, J. Org. Chem., 2012, 77, 2192-2206.

99 A. Doroshenko, A. Kirichenko, V. Mitina and O. Ponomaryov, J. Photochem. Photobiol., A, 1996, 94, 15-26. 100 C. Sissa, A. Painelli, F. Terenziani, M. Trotta and R. Ragni, Phys. Chem. Chem. Phys., 2020, 22, 129-135.

101 D. Huang, Y. Chen and J. Zhao, Dyes Pigm., 2012, 95, 732742.

102 L. Yang, Y. Liu, X. Zhou, Y. Wu, C. Ma, W. Liu and C. Zhang, Dyes Pigm., 2016, 126, 232-238.

103 T. Kushida, C. Camacho, A. Shuto, S. Irle, M. Muramatsu, T. Katayama, S. Ito, Y. Nagasawa, H. Miyasaka and E. Sakuda, Chem. Sci., 2014, 5, 1296-1304.

104 D. Cortecchia, S. Neutzner, A. R. Srimath Kandada, E. Mosconi, D. Meggiolaro, F. De Angelis, C. Soci and A. Petrozza, J. Am. Chem. Soc., 2017, 139, 39-42.

105 A. Yangui, D. Garrot, J.-S. Lauret, A. Lusson, G. Bouchez, E. Deleporte, S. Pillet, E.-E. Bendeif, M. Castro and S. Triki, J. Phys. Chem. C, 2015, 119, 23638-23647.

106 H. Von Berlepsch, C. Böttcher and L. Dähne, J. Phys. Chem. $B, 2000,104,8792-8799$.
107 F. Würthner, T. E. Kaiser and C. R. Saha-Möller, Angew. Chem., 2011, 123, 3436-3473.

108 N. Ryu, Y. Okazaki, E. Pouget, M. Takafuji, S. Nagaoka, H. Ihara and R. Oda, Chem. Commun., 2017, 53, 8870-8873.

109 V. Lau and B. Heyne, Chem. Commun., 2010, 46, 3595-3597.

110 Y. Hong, J. W. Lam and B. Z. Tang, Chem. Soc. Rev., 2011, 40, 5361-5388.

111 M. R. Molla and S. Ghosh, Chem.-Eur. J., 2012, 18, 1290.

112 J. Gong, P. Wei, Y. Su, Y. Li, X. Feng, J. W. Lam, D. Zhang, X. Song and B. Z. Tang, Chin. Chem. Lett., 2018, 29, 1493-1496.

113 A. Prakash and D. Adhikari, Int. J. ChemTech Res., 2011, 3, 1891-1896.

114 H. Song, Y. Zhou, H. Qu, C. Xu, X. Wang, X. Liu, Q. Zhang and X. Peng, Ind. Eng. Chem. Res., 2018, 57, 15216-15223.

115 W. Tang, Y. Xiang and A. Tong, J. Org. Chem., 2009, 74, 2163-2166.

116 L. Q. Yan, Z. N. Kong, Y. Xia and Z. J. Qi, New J. Chem., 2016, 40, 7061-7067.

117 T. Wu, J. Huang and Y. Yan, Chem.-Asian J., 2019, 14, 730750.

118 J. Birks, Nature, 1967, 214, 1187-1190.

119 Y. N. Teo and E. T. Kool, Chem. Rev., 2012, 112, 4221-4245.

120 M.-C. Heissenbüttel, P. Marauhn, T. Deilmann, P. Krüger and M. Rohlfing, Phys. Rev. B: Condens. Matter Mater. Phys., 2019, 99, 035425.

121 A. Cuppoletti, Y. Cho, J.-S. Park, C. Strässler and E. T. Kool, Bioconjugate Chem., 2005, 16, 528-534.

122 A. Kobori, T. Ueda, Y. Sanada, A. Yamayoshi and A. Murakami, Biosci., Biotechnol., Biochem., 2013, 77, 1117-1119.

123 J. Nie, Y. Liu, J. Niu, Z. Ni and W. Lin, J. Photochem. Photobiol., A, 2017, 348, 1-7.

$124 \mathrm{X}$. Li and H. H. Patterson, Materials, 2013, 6, 2595-2611.

125 Y. Shao, G.-Z. Yin, X. Ren, X. Zhang, J. Wang, K. Guo, X. Li, C. Wesdemiotis, W.-B. Zhang and S. Yang, RSC Adv., 2017, 7, 6530-6537.

126 H. Schmidbaur and H. G. Raubenheimer, Angew. Chem., 2020, 14748-14771.

127 X. Ma, J. Wang and H. Tian, Acc. Chem. Res., 2019, 52, 738748.

128 S. Hirata, K. Totani, J. Zhang, T. Yamashita, H. Kaji, S. R. Marder, T. Watanabe and C. Adachi, Adv. Funct. Mater., 2013, 23, 3386-3397.

129 T. Zhang, X. Ma, H. Wu, L. Zhu, Y. Zhao and H. Tian, Angew. Chem., Int. Ed., 2020, 59, 11206-11216.

130 D. Li, F. Lu, J. Wang, W. Hu, X.-M. Cao, X. Ma and H. Tian, J. Am. Chem. Soc., 2018, 140, 1916-1923.

131 Y. Gong, H. Chen, X. Ma and H. Tian, ChemPhysChem, 2016, 17, 1934-1938.

132 T. Li and X. Ma, Dyes Pigm., 2018, 148, 306-312.

133 H. Nerenz, M. Meier, W. Grahn, A. Reisner, E. Schmälzlin, S. Stadler, K. Meerholz, C. Bräuchle and P. G. Jones, J. Chem. Soc., Perkin Trans. 2, 1998, 2, 437-448.

134 X. Li, D. Zhang, G. Lu, G. Xiao, H. Chi, Y. Dong, Z. Zhang and Z. Hu, J. Photochem. Photobiol., A, 2012, 241, 1-7.

135 Q. Zhang, Q. Zhou, Y. Cheng, L. Wang, D. Ma, X. Jing and F. Wang, Adv. Mater., 2004, 16, 432-436. 
136 A. Singh, A. Singh, N. Singh and D. O. Jang, Sens. Actuators, $B, 2017,243,372-379$.

137 S. AlAbbad, T. Sardot, O. Lekashvili, D. Decato, F. Lelj, J. A. Ross and E. Rosenberg, J. Mol. Struct., 2019, 1195, 620-631.

138 J. Tao, D. Sun, L. Sun, Z. Li, B. Fu, J. Liu, L. Zhang, S. Wang, Y. Fang and H. Xu, Dyes Pigm., 2019, 168, 166-174.

139 Y. Liu, L. Yang, C. Ma and A. Tang, Dyes Pigm., 2020, 173, 107981.

140 D. Dahal, L. McDonald, X. Bi, C. Abeywickrama, F. Gombedza, M. Konopka, S. Paruchuri and Y. Pang, Chem. Commun., 2017, 53, 3697-3700.

141 M. S. T. Gonçalves, Chem. Rev., 2009, 109, 190-212.

142 X. Peng, F. Song, E. Lu, Y. Wang, W. Zhou, J. Fan and Y. Gao, J. Am. Chem. Soc., 2005, 127, 4170-4171.

143 V. Sharma, D. Sahoo, N. Varghese, K. Mohanta and A. L. Koner, $R S C$ Adv., 2019, 9, 30448-30452.

144 L. E. McNamara, T. A. Rill, A. J. Huckaba, V. Ganeshraj, J. Gayton, R. A. Nelson, E. A. Sharpe, A. Dass, N. I. Hammer and J. H. Delcamp, Chem.-Eur. J., 2017, 23, 12494-12501.

145 Y. Xu, Q. Liu, X. Li, C. Wesdemiotis and Y. Pang, Chem. Commun., 2012, 48, 11313-11315.

146 X. Liu, B. Cho, L.-Y. Chan, W. L. Kwan and C.-L. K. Lee, $R S C$ Adv., 2015, 5, 106868-106876.
147 C. Liu, X. Jiao, Q. Wang, K. Huang, S. He, L. Zhao and X. Zeng, Chem. Commun., 2017, 53, 10727-10730.

148 A. G. Jadhav, S. Kothavale and N. Sekar, Dyes Pigm., 2017, 138, 56-67.

149 W. Lin, L. Yuan, Z. Cao, Y. Feng and J. Song, Angew. Chem., Int. Ed., 2010, 49, 375-379.

150 S. Kothavale, A. G. Jadhav and N. Sekar, Dyes Pigm., 2017, 137, 329-341.

151 A. Fletcher, D. Bliss and J. M. Kauffman, Opt. Commun., 1983, 47, 57-61.

152 T. Besson, G. Coudert and G. Guillaumet, J. Heterocycl. Chem., 1991, 28, 1517-1523.

153 M. Beija, C. A. Afonso and J. M. Martinho, Chem. Soc. Rev., 2009, 38, 2410-2433.

154 E. Heid, S. Schmode, P. Chatterjee, A. D. MacKerell and C. Schröder, Phys. Chem. Chem. Phys., 2019, 21, 1770317710.

155 W.-J. Zhang, L. Fan, Z.-B. Li, T. Ou, H.-J. Zhai, J. Yang, C. Dong and S.-M. Shuang, Sens. Actuators, B, 2016, 233, 566-573.

156 E. B. Brauns, C. J. Murphy and M. A. Berg, J. Am. Chem. Soc., 1998, 120, 2449-2456.

157 M. Streiter, S. Krause, C. von Borczyskowski and C. Deibel, J. Phys. Chem. Lett., 2016, 7, 4281-4284.

158 Q. Wei, Y. Zhao, Q. Di, J. Liu, M. Xu, J. Liu and J. Zhang, J. Phys. Chem. C, 2017, 121, 6152-6159. 